\title{
Liability Insurance: Equilibrium Contracts under Monopoly and Competition.*
}

\author{
Jorge Lemus ${ }^{\dagger}$ Emil Temnyalov; and John L. Turner ${ }^{\S}$
}

March 30, 2020

\begin{abstract}
In liability lawsuits (e.g., patent infringement) a plaintiff demands compensation from a defendant and the parties often negotiate a settlement to avoid a costly trial. Liability insurance creates bargaining leverage for the defendant in this settlement negotiation. We study the characteristics of monopoly and equilibrium contracts in settings where this leverage effect is a substantial source of value for insurance. Our results show that under adverse selection, a monopolist offers at most two contracts, which under-insure low-risk types and may inefficiently induce high-risk types to litigate. In a competitive market, only a pooling equilibrium with under-insurance may exist.
\end{abstract}

JEL Codes: C7, D82, G22, K1, K41.

Keywords: liability, insurance, litigation, bargaining, adverse selection, competitive equilibrium, monopoly.

${ }^{*}$ We appreciate helpful comments by Dan Bernhardt, Yeon-Koo Che, In-Koo Cho, Simon Grant, Bruno Jullien, Stefan Krasa, Patrick Rey, Guillaume Roger, Art Snow and seminar audiences at IIOC, ESAM, EARIE, LACEA, APIOC, AETW, AMES, Clemson University, Monash University, UIUC, UNSW, Texas A\&M University, Cornell (Johnson), ZJU Conference on Industrial Economics, University of Louisvilly, Iowa State University. We have also benefited from discussions with Jorge Gallardo-Garcia and other liability and litigation experts at Bates White. Turner acknowledges research support from the Terry-Sanford research program and from the James C. Bonbright Center for the Study of Regulation.

$\dagger$ University of Illinois Urbana-Champaign, Department of Economics. jalemus@illinois.edu

$\ddagger$ University Technology Sydney, Business School. emil.temnyalov@uts.edu.au

§University of Georgia, Department of Economics. jlturner@uga.edu. 


\section{Introduction}

Third-party liability insurance protects against liability for harm caused to a third party, whereas first-party insurance protects against own losses (e.g. health or life insurance). Under third-party insurance, claims for damages require a court trial to determine whether the insured agent is liable for the loss incurred by the third party. Most liability lawsuits, however, are settled out of court, to avoid litigation costs. Liability insurance is valuable in part because it gives the insured agent leverage when bargaining with the third party over settlement. Insurance raises the agent's payoff from litigation, making the agent more willing to litigate, and lowers the payment to the third party if there is a settlement. This leverage effect makes third-party insurance valuable even for risk-neutral agents, in contrast to first-party insurance where there is no possibility to bargain over the potential loss.

This paper is the first to study the economics of such leverage under adverse selection. We study equilibrium contracts under different information and market structures, modeling a negotiation framework with endogenous payoffs determined in a rich contracting environment for third-party insurance. ${ }^{1}$ In our setting, a risk neutral agent buys an insurance contract that covers a portion of the litigation costs and/or damages, if the agent goes to litigation, and a portion of the settlement transfer, if the agent and the third party settle. At the time of contracting, the agent may or may not know her type, which is the probability of being found liable in court. If and when a third party subsequently sues the agent for damages, the agent and third party bargain over a settlement under the threat of litigation. By covering litigation expenses, insurance reduces the agent's pressure to settle, so parties either settle at a lower settlement fee (relative to no insurance) or they litigate. The contract offered by the insurer must balance two effects: a more generous policy increases the agent's willingness to pay for the contract, but may incentivize costly litigation.

In our setting, the agent's willingness to pay for insurance is generally non-differentiable and the cost of providing insurance is generally discontinuous. The non-differentiability and discontinuity properties arise because the agent has a non-contractible ex-post action-to settle or litigate - that introduces ex-post moral hazard; these features do not appear in first-

\footnotetext{
${ }^{1}$ The effect of insurance on the agent's ability to negotiate a settlement also relates to the literature on third-party funding of plaintiffs and its effect on settlement (Daughety and Reinganum, 2014).
} 
party insurance because there is no effect of insurance on negotiation incentives. Our findings show that the canonical model for insurance applies only to first-party insurance; third-party liability insurance requires a richer model that also considers the effect of insurance on an agent's ex post bargaining incentives. As a result, we find that equilibrium liability contracts differ strongly from standard results on first-party insurance contracts for both monopoly and perfect competition.

First, with a single seller and regular type distributions, we find that the optimal menu only partially covers the damage payments of high-risk types, generally features distortions at the top, in addition to distortion at the bottom, and it does not necessarily allocate perfect insurance to the highest type. These findings contrast with standard results on first-party insurance (see, e.g., Stiglitz (1977), Chade and Schlee (2012)). We find that in any optimal mechanism at most two classes of contracts are offered in equilibrium-one that does not cover damages, targeting low-risk types, and one that partially covers damage payments, aimed at high-risk types; the optimal contracts always cover legal costs and do not cover settlements (a policy known as "legal expenses insurance" in practice). ${ }^{2}$ Insurance covering settlements directly is not optimal because it weakens the agent's leverage in bargaining. Insurance does add value to settlements indirectly, through an off-path equilibrium leverage effect: by covering litigation expenses, the insurance improves the agent's bargaining position and reduces the equilibrium settlement fee. In some cases, the optimal contract may induce inefficient litigation in equilibrium, whereas in the absence of insurance there would not have been litigation. This points to novel potentially negative welfare effects of liability insurance.

Second, in a competitive market under asymmetric information, we find that for any distribution of types there can only be pooling equilibria. Any such equilibrium never induces litigation and features under-insurance. These results contrast with the seminal work of Rothschild and Stiglitz (1976), where only separating contracts are offered in a competitive equilibrium with discrete type distributions, and no equilibrium exist with a continuum of types (Riley, 1979). In Rothschild and Stiglitz (1976), in a candidate pooling equilibrium, an insurer is able to profitably deviate by offering a contract that only attracts types who generate positive surplus, which undermines the cross-subsidization needed to sustain the

\footnotetext{
${ }^{2}$ Chade and Schlee (2019) show that provision costs can generate pooling in first-party insurance.
} 
pooling equilibrium.

With third-party insurance, the cost discontinuity facing the insurer, driven by the agent's decision to settle or to litigate, plays a crucial role in the equilibrium analysis. We show that the insurer's costs are zero when the agent settles, and strictly positive and increasing with the agent's type when the agent litigates. As a consequence, cross-subsidization is not necessary as long as insurance does not induce litigation. Each type that settles costs the insurer the same, which enables pooling to survive in equilibrium for both discrete and continuous distributions. A separating equilibrium requires that contracts be sold at different prices, because otherwise types would pool on the more generous insurance. But for two contracts to yield zero profit, at least one contract must attract types that settle and types that litigate. Such a contract cannot survive in equilibrium, because insurers incur a loss on types that litigate so it requires cross-subsidization between types that settle and types that litigate. It is therefore "cream-skimmed" by another contract that only attracts types that settle. This implies that a separating equilibrium does not exist. Similar to Rothschild and Stiglitz (1976), however, we find that adverse selection destroys the possibility of equilibrium altogether when there are too few high-risk agents. Subsequent work - e.g., Wilson (1977); Miyazaki (1977); Riley (1979); Crocker and Snow (1985); Azevedo and Gottlieb (2017); Farinha Luz (2017) shows that alternative equilibrium concepts change both the set of equilibrium contracts and welfare implications. Pooling can arise in equilibrium in first-party insurance by incorporating economies of scale (Allard et al., 1997) or transaction costs (Ramsay et al., 2013).

We also provide results on the effect of liability insurance on litigation: under incomplete information, optimal and equilibrium insurance policies induce more litigation when information is asymmetric; under complete information, insurance never induces litigation.

To focus on the leverage effect of liability insurance, our core model assumes risk neutrality and allocates to the agent control over the decision to litigate or settle. These assumptions closely capture defensive patent litigation insurance, where the policy holder often has such control. $^{3}$ For instance, in the United States, the patent litigation insurance industry has

\footnotetext{
${ }^{3}$ E.g., see marketing materials of IPISC "[...] the Named Insured is in control of the lawsuit." https://keh4ins.com/wp-content/uploads/2014/04/defense-insurance-product-packet-ipisc.pdf; Blue Iron, "With either patent enforcement or patent defense policies, you control the litigation." https://blueironip.com/ip-insurance-by-blueiron/; or Upcounsel "As the policy holder, you will control the lawsuit." https://www.upcounsel.com/patent-infringement-insurance-cost. (Visited on November 2019)
} 
been led by Intellectual Property Insurance Services Corporation (IPISC), an insurer that has marketed its insurance products specifically to firms wishing to make themselves more formidable in litigation. ${ }^{4}$ The worldwide market for patent infringement lawsuit insurance has grown to include at least eight carriers, all of which sell infringement defense coverage and many of which sell multiple products (Gauntlett 2019, Appendix J). ${ }^{5}$

In the broader liability insurance market, most policies cover legal costs and some cover damages/settlements. Past liability claims are usually excluded from coverage. Schwarcz and Siegelman (2017) presents additional examples of liability insurance contracts in different industries. In some types of liability insurance, the insurer controls settlement (e.g., medical malpractice). Meurer (1992) and Sykes (1994), among others, have shown that this creates a potential conflict of interest between the insurer and insured. The leverage effect of insurance also emerges with insurer control (Meurer, 1992), so our results have implications for such policies as well. Moreover, many policies where settlement is delegated to the insurer feature a "consent to settle" clause that effectively gives the agent control over the decision to litigate or settle (e.g. in professional liability, errors and omissions liability and executive liability).

Finally, we discuss additional economic forces at play when risk-averse agents purchase insurance. Risk-averse agents value damages coverage more than risk-neutral agents because, apart from the leverage effect, they value the risk protection against the uncertain litigation outcome. Although increasing coverage for damages increases risk-averse agents' willingness to pay for insurance, it also incentivizes them to litigate, which may be costly for the insurer. To balance these opposing effects, the insurer may require to cover settlements, which breaks up monotonicity properties of the risk-neutral setting. Despite our model being analytically intractable in a general framework, we show that for mean-variance preferences and low levels of risk aversion our main results under perfect competition hold; under monopoly, the contracting space expands because risk aversion introduces more curvature in the utility function. It remains true that low-risk agents are sold a policy that only covers legal costs, high-risk agents are sold a policy that covers legal costs and damages, and no agents receive

\footnotetext{
${ }^{4}$ IPISC's Defense Insurance Program Summary (see Footnote 3) highlights how the product is marketed, including such advantages as "Reduces the pressure of entering into an undesirable license agreement or the risk of incurring burdensome royalty payments" and "Reduces the pressure to settle Infringement cases."

${ }^{5}$ This appendix lists twenty-six products sold by AIG, CFC, IPISC, Liberty Specialty Markets, OPUS, RPX, Safeonline, and Tokio Marine Kiln. It also highlights differences across policies with respect to coverage for pre-existing infringement, dishonest acts, damages coverage, and breach of contract by licensees.
} 
settlement coverage. Hence the leverage value of insurance remains the dominant factor for low levels of risk aversion. ${ }^{6}$

\section{Related Literature}

A large literature on equilibrium contracts for first party insurance, pioneered by Rothschild and Stiglitz (1976) and Stiglitz (1977), includes Wilson (1977); Miyazaki (1977); Riley (1979); Crocker and Snow (1985); Azevedo and Gottlieb (2017); Farinha Luz (2017); Allard et al. (1997); Ramsay et al. (2013); Chade and Schlee (2012). None of these articles, however, study third-party insurance, where bargaining leverage plays a prominent role.

Several articles focus on how liability insurance affects the incentives to invest in prevention (e.g., Shavell, 1982, 2005; Chen and Hua, 2012), ignoring the ex-post bargaining stage between the insured agent and a third party. Another part of the literature incorporates the ex-post bargaining stage between the insured agent and a third party, but focuses on the insurer's incentives to control the litigation process, in the absence of adverse selection (Meurer, 1992; Sykes, 1994). We advance the literature by studying how the bargaining leverage gained by an insured agent affect equilibrium contracts for liability insurance.

The driving economic force in our paper relates to the literature on third-party funding of plaintiffs and its effect on settlement. For example, Daughety and Reinganum (2014) adapt the signaling model of Reinganum and Wilde (1986) to show that optimal loans to privatelyinformed plaintiffs may both eliminate the possibility of litigation and extract favorable settlement terms. Also related, the literature on offensive patent insurance shows that some litigation threats become credible under insurance, which increases the entry deterrence value of patents. Llobet and Suarez (2012) and Buzzacchi and Scellato (2008) study insurance that covers a fraction of the patentee's litigation costs. Duchene (2015) shows that with private information, patent holders may opt not to buy insurance because of an inability to signal and avoid pooling equilibria. In our setting, our assumption of risk-neutrality helps to isolate and highlight the leverage effect of third-party insurance. Shavell (2005), among others, also study liability insurance in a setting where parties are risk-neutral - which is also justified

\footnotetext{
${ }^{6}$ Our Online Appendix presents more details on the issues of risk aversion, endogenous lawsuit control, bargaining under asymmetric information, and supplementary technical results for the baseline model.
} 
by the fact that in many markets liability insurance is primarily bought by firms.

Our paper is also related to the literature on optimal contracting under adverse selection and moral hazard (Picard, 1987; Guesnerie et al., 1989). The key driving force in our model is the improved bargaining position of an insured agent. Kirstein (2000), Van Velthoven and van Wijck (2001), Kirstein and Rickman (2004), and Llobet and Suarez (2012) have shown that risk-neutral buyers may value insurance because it makes litigation credible or it improves the policy holder's bargaining position. However, none of these papers study equilibrium under adverse selection or the optimal monopoly contract. Townsend (1979) also shows that contracts change when it is costly to verify an agent's private information. In our setting, the agent does not know the true state of liability and its verification requires costly litigation.

Finally, our work also relates to the literature on lawyers' contingent fees, where lawyers charge lower upfront fees but keep part of any payments awarded. Dana and Spier (1993) show that contingency fees help solve an agency problem. Rubinfeld and Scotchmer (1993) study a Rothschild-Stiglitz-style model and show that clients with high-quality cases can signal their cases' strength by selecting hourly fees, while attorneys can signal their ability by requesting contingency fees. Gravelle and Waterson (1993) make similar points. Finally, Hay and Spier (1998) and Spier (2007) review the large literature on litigation and settlement.

\section{Model}

A risk-neutral agent $(A)$ sells a product or provides a service that may harm a risk-neutral third party $(T P)$, thereby creating a legal liability. Only a costly trial can verify if $T P$ was harmed by $A$ 's product. $A$ and $T P$ trial $\operatorname{costs}$ are $c_{A}$ and $c$, respectively. The probability that $A$ is found liable ( $A$ 's type) is $p \in[0,1]$ drawn from a distribution $F$ with density $f$. If the court rules that TP was harmed, $A$ must compensate $T P$ with a payment of $d$. The agent can purchase a third-party liability insurance contract from a profit-maximizing risk-neutral insurer $(I)$. Under contract $\alpha=\left(\alpha_{S}, \alpha_{L}, \alpha_{D}\right), I$ pays $A$ up to $\alpha_{S}$ to cover any settlement transfer (if $A$ and TP settle), $\alpha_{L}$ to cover the litigation costs, and $\alpha_{D}$ to cover damages (if the trial finds $A$ is liable). Our goal is to derive the equilibrium contracts offered by $I$, from 
the set of contracts $\mathcal{A}=\left\{\alpha=\left(\alpha_{S}, \alpha_{L}, \alpha_{D}\right): \alpha_{S} \geq 0, \alpha_{L} \leq c_{A}, \alpha_{D} \leq d\right\},{ }^{7}$ for different market and information structures.

Figure 1 describes the timing of the model. At $t=1, I$ offers an insurance contract to $A$. $A$ 's type may be known or unknown by $A$ or $I$ at the time of contracting. Even when $p$ is known to the agent, this may or may not be verifiable information for the insurer. If $p$ is known to the agent and verifiable by the insurer, then $A$ and $I$ contract under complete information (Proposition 2). When $p$ is known to the agent but it is non-verifiable for the insurer, $A$ and $I$ contract under adverse selection (Section 3.2). Finally, both $A$ and $I$ may only know that $p \sim F$ at the time of contracting (Section 3.3). ${ }^{8}$

\begin{tabular}{lccc}
\multicolumn{1}{c}{$t=1$} & $t=2$ & $t=3$ & $t=4$ \\
\hline offers liability & $T P$ sues $A$ if $p d \geq c$ & $A$ and $T P$ negotiate to & If there is no settlement, \\
insurance to $A$ & $(p$ publicly revealed $)$ & avoid trial and to settle & $A$ and $T P$ go to trial
\end{tabular}

Figure 1: Timing of the events in the model.

We assume that $A$ 's type is known by $T P$, and it becomes public when the lawsuit is filed. Thus, $A$ and TP bargain under complete information in a litigation game that starts at $t=2$. At $t=2, T P$ has a credible litigation threat if and only if $p d \geq c$. If $p d<c$, the game ends. If and when a lawsuit is filed, $A$ and $T P$ bargain under complete information (at $t=3$ ) over a settlement fee under the Nash bargaining protocol. A's bargaining skill in this negotiation is $\theta \in(0,1)$. If a settlement is not reached, then a trial ensues $(t=4) .^{9}$

Importantly, A's disagreement payoff (i.e., A's expected litigation payoff) depends both on the probability of liability $p$ and on the insurance contract $\alpha$ bought by the agent: $V_{L}(p, \alpha) \equiv$ $-c_{A}-p d+\alpha_{L}+p \alpha_{D}$. Insurance improves $A$ 's disagreement payoff by $\alpha_{L}+p \alpha_{D}$, strengthening A's bargaining position in the settlement negotiation. This bargaining leverage distinguishes third-party insurance from first-party insurance.

We analyze both the optimal liability-insurance contracts offered by a monopolist and the

\footnotetext{
${ }^{7}$ The restriction $\alpha_{L} \leq c_{A}$ and $\alpha_{D} \leq d$ precludes over-insurance. In practice many insurance contracts are implemented as a reimbursement policy, so over-insurance is indeed impossible.

${ }^{8}$ In this case, the insurer cannot renegotiate the contract signed at $t=1$ (e.g. renegotiation is expensive). If contracts were renegotiable, then the solution is equivalent to selling insurance under complete information (if $p$ is verifiable) or under adverse selection (if $p$ is non-verifiable).

${ }^{9}$ Note that in principle, A and TP could collude in sham litigation that would defraud the insurer. We assume that such fraudulent action is deterred by large expected penalties (e.g., fines, costs, or jail time).
} 
equilibrium liability-insurance contracts offered by a competitive market under adverse selection. Before we proceed with this analysis, we first present some preliminary analysis and derive by backward induction $A$ 's willingness to pay and I's cost of providing insurance.

Note that when $A$ is uninsured, then $A$ and $T P$ always settle because their net joint surplus from a settlement is positive $\left(c_{A}+c>0\right)$. When $A$ is insured, however, settlement does not necessarily occur. If $A$ is covered by insurance policy $\alpha \in \mathcal{A}$, and TP receives a transfer $T$ to settle, then $A$ and $T P^{\prime}$ 's net joint surplus from settling is

$$
\begin{aligned}
S_{B} & =\underbrace{\min \left\{-T+\alpha_{S}, 0\right\}}_{\begin{array}{c}
\text { A's settlement } \\
\text { payoff }
\end{array}}+\underbrace{T}_{\begin{array}{c}
T P^{\prime} \text { s settlement } \\
\text { payoff }
\end{array}}-[\underbrace{V_{L}(p, \alpha)}_{\begin{array}{c}
A^{\prime} \text { 's litigation } \\
\text { payoff }
\end{array}}+\underbrace{p d-c}_{\begin{array}{c}
T P^{\prime} \text { s litigation } \\
\text { payoff }
\end{array}}], \\
& =\min \left\{\alpha_{S}, T\right\}+c+c_{A}-\alpha_{L}-p \alpha_{D} .
\end{aligned}
$$

The bargaining surplus in Equation 1 could be negative, depending on the insurance policy and $A$ 's type: $\alpha_{L}$ and $\alpha_{D}$ reduce it and $\alpha_{S}$ increases it. Any contract $\alpha$ determines two threshold types that are relevant for our analysis. Denote $p^{*}(\alpha)$ as the litigation-threshold type: types $p \leq p^{*}(\alpha)$ settle $\left(S_{B} \geq 0\right)$ and types $p>p^{*}(\alpha)$ litigate $\left(S_{B}<0\right)$. Denote $p^{* *}(\alpha)$ as the full-settlement-coverage-threshold type: types $p \leq p^{* *}(\alpha)$ have their settlement offer fully covered by $I$. For types in $\left[p^{* *}(\alpha), p^{*}(\alpha)\right]$ the settlement offer is not entirely covered by insurance. ${ }^{10,11}$

Proposition 1. The willingness to pay of an agent of type $p$ for insurance policy $\alpha \in \mathcal{A}$ is

$$
W(p, \alpha)= \begin{cases}p d-c+(1-\theta)\left(c+c_{A}\right) & \text { if } \frac{c}{d} \leq p<\max \left\{p^{* *}(\alpha), \frac{c}{d}\right\} \\ \theta \alpha_{S}+(1-\theta)\left(\alpha_{D} p+\alpha_{L}\right) & \text { if } \max \left\{p^{* *}(\alpha), \frac{c}{d}\right\} \leq p \leq p^{*}(\alpha) \\ \alpha_{D} p+\alpha_{L}-\theta\left(c+c_{A}\right) & \text { if } p>p^{*}(\alpha)\end{cases}
$$

\footnotetext{
${ }^{10}$ To avoid confusion with notation, note that the asterisks in $p^{*}$ and $p^{* *}$ denote threshold types as a function of contract features, not optimality. Whereas in subsequent sections, when we characterize contracts in various settings, optimal contract features will be denoted with $p_{i, j}^{*}$, where $i \in\{M, C\}$ distinguishes the monopoly and competition settings while $j \in\{A I, S I\}$ denotes asymmetric and symmetric information.

${ }^{11}$ All omitted proofs are in Appendix A.
} 
The insurer's expected cost for providing insurance policy $\alpha$ to an agent of type $p$ is

$$
K(p, \alpha)=\left\{\begin{array}{ll}
\alpha_{S} & \text { if } p \leq p^{*}(\alpha) \\
\alpha_{D} p+\alpha_{L} & \text { if } p>p^{*}(\alpha)
\end{array},\right.
$$

where $p^{*}(\alpha) \equiv \frac{\alpha_{S}+c+c_{A}-\alpha_{L}}{\alpha_{D}}$, and $p^{* *}(\alpha) \equiv \frac{\theta\left(\alpha_{S}+c\right)-(1-\theta)\left(c_{A}-\alpha_{L}\right)}{d-\alpha_{D}(1-\theta)}$.

Figure 2 illustrates Proposition 1 for the case where $\frac{c}{d}<p^{* *}(\alpha)<p^{*}(\alpha)$. The willingness to pay $W(p, \alpha)$ is monotone increasing, continuous, and piecewise linear in $p$. The cost $K(p, \alpha)$ has a discontinuity at the litigation threshold $p^{*}(\alpha)$, and jumps from a value strictly below $W\left(p^{*}(\alpha), \alpha\right)$ to a value strictly above it, i.e., $K\left(p^{*}(\alpha), \alpha\right)<W\left(p^{*}(\alpha), \alpha\right)<K\left(p^{*}(\alpha)^{+}, \alpha\right)$.

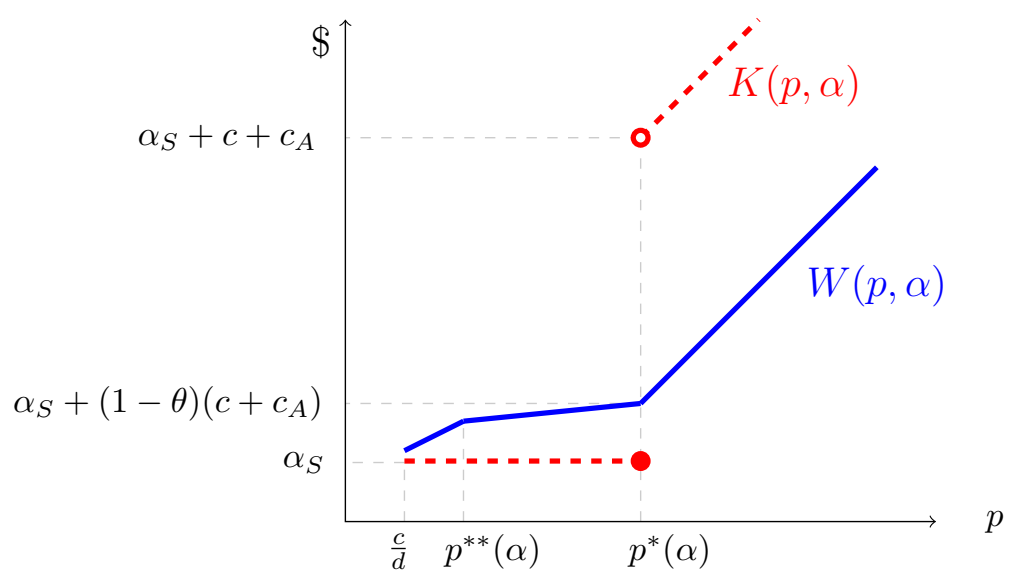

Figure 2: The solid line is type $p$ 's willingness to pay for insurance policy $\alpha, W(p, \alpha)$. The dashed line is the insurer's cost of providing policy $\alpha$ for type $p, K(p, \alpha)$. Types above $p^{*}(\alpha)$ litigate and types below $p^{*}(\alpha)$ settle. The settlement fee is fully paid by the insurer for types below $p^{* *}(\alpha)$.

Subtracting Equation 3 from Equation 2, we find $A$ and $I$ 's net joint surplus,

$$
W(p, \alpha)-K(p, \alpha)= \begin{cases}p d-c+(1-\theta)\left(c+c_{A}\right)-\alpha_{S} & \text { if } \frac{c}{d} \leq p \leq p^{* *}(\alpha), \\ (1-\theta)\left(\alpha_{D} p+\alpha_{L}\right)-(1-\theta) \alpha_{S} & \text { if } p^{* *}(\alpha)<p \leq p^{*}(\alpha), \\ -\theta\left(c+c_{A}\right) & \text { if } p>p^{*}(\alpha) .\end{cases}
$$

Equation 4 highlights how the value of third-party insurance depends upon bargaining and settlement. First, insurance does not improve the bargaining ability of agents whose settlement payment is fully-covered by $I$ : For $p \in\left[\frac{c}{d}, p^{* *}(\alpha)\right], T P$ fully extracts $A$ 's settlement 
coverage $\alpha_{S}$; $A$ 's willingness to pay for insurance is what $A$ would have paid had $A$ negotiated uninsured. Second, insurance makes agents better negotiators when they settle and have to pay part of the settlement fee out of pocket: For $p \in\left(p^{* *}(\alpha), p^{*}(\alpha)\right]$, by improving $A^{\prime}$ s outside option, the leverage effect of insurance avoids TP from collecting $(1-\theta)\left(p \alpha_{D}+\alpha_{L}\right)$. The settlement coverage (paid by $I$ ) increases the bargaining surplus by $\alpha_{S}$, from which $A$ recovers a fraction $\theta$. Third, $A$ and $I$ jointly lose the share of bargaining surplus that an uninsured agent extracts from $T P$ in a settlement negotiation.

Note that insuring an agent with perfect bargaining skill $(\theta=1)$ is unprofitable because $W(p, \alpha) \leq K(p, \alpha)$ for all $p$ and for any $\alpha$. Such an agent extracts all the surplus from the settlement negotiation and hence does not need leverage. On the other hand, insuring agents with imperfect bargaining skill $(\theta<1)$ who settle litigation is profitable. The leverage effect of third-party insurance is valuable even if the agents are risk-neutral. ${ }^{12}$

\section{Contracting at $t=1$}

We consider both monopoly and competitive insurance markets, as well as different information structures. The motivation for multiple information environments is differences in the types of risks firms may face. For example, firms that face a small set of specific and known risks may have better information than the insurer. On the other hand, when firms face a large set of unknown risks, information may be close to symmetric between agent and insurer. ${ }^{13}$ Before we proceed, we first characterize the optimal contract under complete information: For each $p$, we look for a contract $\alpha(p)$ that maximizes $W(p, \alpha(p))-K(p, \alpha(p))$.

Proposition 2 (Complete Information). For a monopoly or under perfect competition, if $p$ is observable at the time of contracting, then any contract $\alpha$ such that $p^{*}(\alpha)=p$ is optimal.

Proof. Any contract $\alpha$ such that $p^{*}(\alpha)=p$ maximizes the difference $W(p, \alpha)-K(p, \alpha)$.

\footnotetext{
${ }^{12}$ In extensions of our basic model, we show that the leverage effect of insurance remains the dominant force when the agent is moderately risk averse (see Section 4 and Online Appendix Section B.1).

${ }^{13}$ Within defensive patent litigation insurance, the known-risks (informed A) case more closely captures risks of lawsuits by direct competitors, while the unknown-risks case more closely captures risks of lawsuits by "patent trolls."
} 
Proposition 2 shows that under complete information any contract $\alpha \in \mathcal{A}$ such that $p \alpha_{D}=$ $\alpha_{S}+c+c_{A}-\alpha_{L}$ is optimal. For any such contract, $A$ and $T P$ settle, and $T P$ earns exactly its disagreement payoff, $p d-c$. This is the same outcome of a settlement negotiation between $T P$ and an uninsured agent with perfect bargaining skill $(\theta=1)$. Thus, under complete information, the equilibrium insurance contract extracts all the bargaining surplus from $T P$, and it transfers rents from $T P$ to $I$ (in the case of monopoly) or to $A$ (in the case of perfect competition). We refer to any contract $\alpha$ such that $p^{*}(\alpha)=p$ as perfect insurance for type $p$, because its leverage effect generates the most joint surplus to be shared by $A$ and $I$.

\subsection{Contracting under Adverse Selection and Monopoly}

Consider a profit-maximizing monopolist who offers a mechanism to an agent who holds private information about her type. By the revelation principle we restrict attention to direct mechanisms that are incentive compatible.

A mechanism consists of a menu of contracts $\alpha(\tilde{p})=\left(\alpha_{S}(\tilde{p}), \alpha_{L}(\tilde{p}), \alpha_{D}(\tilde{p})\right)$ and prices $T(\tilde{p})$, which are functions of the agent's reported type $\tilde{p}$. The monopoly insurer chooses the functions $\alpha:\left[\frac{c}{d}, 1\right] \rightarrow[0, \infty) \times\left[0, c_{A}\right] \times[0, d]$ and $T:\left[\frac{c}{d}, 1\right] \rightarrow[0, \infty)$ to maximize:

$$
\max _{\alpha(\cdot), T(\cdot)} \int_{\frac{c}{d}}^{1}[T(p)-K(p, \alpha(p))] f(p) d p
$$

subject to incentive compatibility (IC) and individual rationality (IR). The IC constraint is $p \in \arg \max _{\tilde{p} \in\left[\frac{c}{d}, 1\right]} W(p, \alpha(\tilde{p}))-T(\tilde{p})$. Let $U(p) \equiv \max _{\tilde{p} \in\left[\frac{c}{d}, 1\right]} W(p, \alpha(\tilde{p}))-T(\tilde{p})$. The IR constraint corresponds to $U(p) \geq 0$ for all $p$.

Recall that the agent's willingness to pay (Equation 2) is piecewise linear and therefore not differentiable, and that the insurer's cost function is discontinuous. These issues can in principle invalidate the usual mechanism design approach, because the mechanism may assign a non-zero measure of types to contracts that make them indifferent between settling and litigating. If so, the envelope theorem approach would fail. ${ }^{14}$ However, Lemma 4 (in the Appendix) shows that any incentive compatible mechanism in our setting in fact allocates a

\footnotetext{
${ }^{14}$ Carbajal and Ely (2013) show that in quasi-linear settings with non-differentiable valuations the envelope theorem characterization may lead to a range of possible payoffs as a function of the allocation rule.
} 
measure-zero set of types to this non-differentiable point where $p=p^{*}(\alpha(p))$, which allow us to apply the envelope theorem to derive the optimal mechanism. We have,

$$
T(p)=W(p, \alpha(p))-\int_{\frac{c}{d}}^{p} \frac{\partial W(s, \alpha(s))}{\partial p} d s-U\left(\frac{c}{d}\right)
$$

Replacing this expression in the objective function, noting that the monopolist sets $U\left(\frac{c}{d}\right)=0$, and using the standard change of variables to re-write the information rents term, we obtain:

$$
\max _{\alpha(\cdot)} \int_{\frac{c}{d}}^{1}\left[W(p, \alpha(p))-K(p, \alpha(p))-\frac{\partial W(p, \alpha(p))}{\partial p}\left(\frac{1-F(p)}{f(p)}\right)\right] f(p) d p
$$

In the standard mechanism design problem, incentive compatibility requires an increasing allocation. Although we have a 3-dimensional allocation $\alpha$, the agent's private information is single-dimensional. Lemma 5 (in the Appendix) shows that IC is equivalent to a single monotonicity condition: $\alpha_{D}(p)$ must be non-decreasing.

In solving the insurer's problem, we make the following assumption to restrict attention to cases where types below a certain level are excluded from damage insurance.

Assumption 1. $p-\frac{1-F(p)}{f(p)}$ satisfies single-crossing and crosses zero from below.

Under Assumption 1 we define $\bar{p}$ as the unique solution of

$$
\bar{p}=\frac{1-F(\bar{p})}{f(\bar{p})} .
$$

With our characterization of incentive compatibility, we can follow the usual mechanism design approach. We first maximize the insurer's objective pointwise, for each type, ignoring the monotonicity constraint on $\alpha$. The solution to this pointwise maximization is $\alpha(p)=$ $\left(0, c_{A}, 0\right)$ for $p \leq \bar{p}$ and $\alpha(p)=\left(0, c_{A}, c / p\right)$ for $p>\bar{p}$. Note, however, that this solution does not satisfy the monotonicity condition. Thus, we need to "iron" the pointwise solution to ensure this constraint is satisfied. The following theorem characterizes the optimal menu of contracts offered by a monopolist facing a privately informed agent. 
Theorem 1. Assume the distribution F satisfies Assumption 1 and define

$$
\begin{aligned}
p_{M, A I}^{*} \in \underset{\hat{p}^{*} \in[\bar{p}, 1]}{\arg \max } \Psi_{A I}\left(\hat{p}^{*}\right) & \equiv(1-\theta) c_{A} F(\bar{p})+(1-\theta) \int_{\bar{p}}^{\hat{p}^{*}}\left[c_{A}+\frac{c}{\hat{p}^{*}}\left(p-\frac{1-F(p)}{f(p)}\right)\right] f(p) d p \\
& -\int_{\hat{p}^{*}}^{1}\left[\theta\left(c+c_{A}\right)+\frac{c}{\hat{p}^{*}}\left(\frac{1-F(p)}{f(p)}\right)\right] f(p) d p .
\end{aligned}
$$

The optimal menu of contracts offered by a monopolist insurer consists of two contracts:

1) $\alpha(p)=\left(0, c_{A}, 0\right)$ sold at price $T(p)=(1-\theta) c_{A}$ to types $p \leq \bar{p}$;

2) $\alpha(p)=\left(0, c_{A}, \frac{c}{p_{M, A I}^{*}}\right)$ sold at price $T(p)=(1-\theta)\left(c_{A}+c \frac{\bar{p}}{p_{M, A I}^{*}}\right)$ to types $p>\bar{p}$.

Theorem 1 shows that low-risk types $(p<\bar{p})$ purchase a contract that covers only litigation costs. In practice, such contracts are common and are called "legal expenses insurance." All higher-risk types $(p>\bar{p})$ purchase zero coverage for settlements, full coverage for litigation costs, and a single level of damages coverage. The particular level of damages coverage trades off improved bargaining leverage (for types that buy damages coverage and settle litigation) versus litigation expenses (for types that litigate). ${ }^{15}$ In practice, some carriers offer contracts that do not cover settlements, while others offer contracts that cover settlements but are subject to a deductible. Although we do not model deductibles explicitly, we can interpret contract $\alpha$ as offering coverage $\alpha_{D}$ of both damages and settlement payments, but with a deductible $d-\alpha_{D}$. Under this interpretation, the equilibrium settlement transfer is always lower than the deductible, so the insurer effectively does not cover settlements. ${ }^{16}$

To see the intuition behind the condition in Theorem 1, consider each term in $\Psi_{A I}\left(\hat{p}^{*}\right)$. First, type $\bar{p}$ partitions types into those with positive and with negative virtual surplus. Unlike the standard setting, where the mechanism excludes types with negative surplus, in our setting 'exclusion' refers to exclusion from covering damages. Covering litigation costs gives agents a type-independent bargaining leverage for which low-risk $(p<\bar{p})$ agents are willing to pay $(1-\theta) c_{A}$. Thus, the monopolist sells the contract $\alpha(p)=\left(0, c_{A}, 0\right)$ to these types at price $(1-\theta) c_{A}$, and receives an aggregate profit of $(1-\theta) c_{A} F(\bar{p})$. This is the first term in $\Psi_{A I}\left(\hat{p}^{*}\right)$.

For high-risk types (types above $\bar{p}$ ), the insurer offers a contract with the same levels of

\footnotetext{
${ }^{15}$ As we discuss in Section 4, these features of the optimal menu hinge on the risk-neutrality assumption.

${ }^{16}$ For any contract $\alpha$ with corresponding litigation threshold $p^{*}(\alpha)$, the settlement transfer is lower than $d-\frac{c}{p^{*}(\alpha)}$, for all $p \leq p^{*}(\alpha)$, iff $0 \leq\left(d p^{*}(\alpha)-c\right)(1-p)+c \theta\left(p^{*}(\alpha)-p\right)$, which holds for $\frac{c}{d} \leq p \leq p^{*}(\alpha)$.
} 
coverage for litigation costs and settlement than the contract sold to low-risk types, but it also partially covers damages. Conditional on $\alpha_{S}=0$ and $\alpha_{L}=c_{A}$, setting $\alpha_{D}=\frac{c}{p}$ corresponds to perfect insurance for type $p$ (see Proposition 2), but offering perfect insurance to these types is not incentive compatible (Lemma 5): $\alpha_{D}(\cdot)$ would be strictly decreasing in $p$. Ironing this solution allocates to all types $p>\bar{p}$ the same level of damages insurance. We can write this coverage as $\alpha_{D}=\frac{c}{\hat{p}^{*}}$, which corresponds to perfect insurance for type $\hat{p}^{*}$. The second term in $\Psi_{A I}\left(\hat{p}^{*}\right)$ captures profits from types in $\left[\bar{p}, \hat{p}^{*}\right)$, who purchase this contract and settle litigation. The insurance contract does not extract all the bargaining surplus from the third party, because for $p<\hat{p}^{*}$, damages insurance is below the perfect level. The third term in $\Psi_{A I}\left(\hat{p}^{*}\right)$ captures losses from types in $\left(\hat{p}^{*}, 1\right]$, who litigate. Each of these types generates a loss of $\theta\left(c+c_{A}\right)$ in joint surplus between the insurer and the agent.

In optimizing $\Psi_{A I}\left(\hat{p}^{*}\right)$, the monopolist chooses a litigation threshold. Figure 3 illustrates this trade-off. Area A represents the monopolist's profit from selling "legal expenses insurance," i.e. not damages insurance, to types below $\bar{p}$. Area $\mathrm{B}$ represents the deadweight loss from excluding these types from damages insurance. Area $\mathrm{C}$ represents the insurer's revenue from contract $\hat{p}^{*}$ sold to types in $\left[\bar{p}, \hat{p}^{*}\right]$. Area D represents the information rents these types obtain. Types in $\left[\frac{c}{d}, \hat{p}^{*}\right]$ settle, and are under-insured, except for $\hat{p}^{*}$ who receives perfect insurance. Types above $\hat{p}^{*}$ are over-insured. Areas $\mathrm{E}+\mathrm{F}$ represent the total net loss incurred by the insurer, net of the revenue from the price paid by types in $\left[\hat{p}^{*}, 1\right]$ : $\mathrm{E}$ is forgone revenue from the information rents; $\mathrm{F}$ is an efficiency loss from litigation. The optimal litigation threshold $p_{M, A I}^{*}$ in Theorem 1 optimizes this tradeoff accounting for the distribution of types.

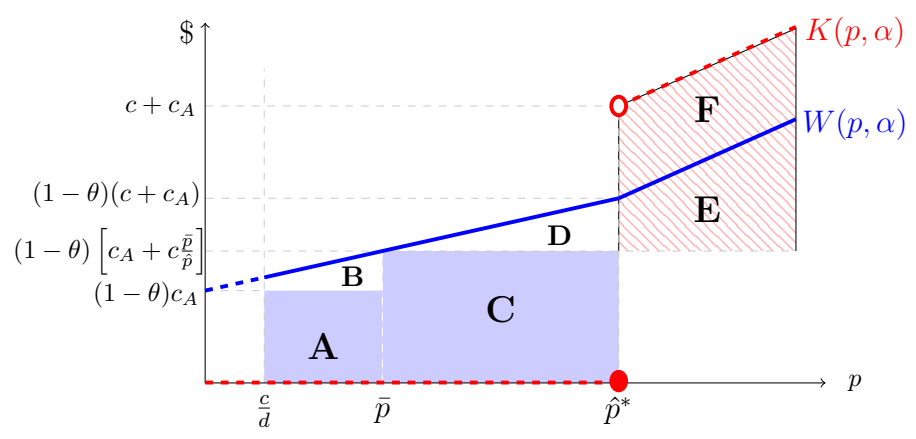

Figure 3: Insurer's gains (solid area) and losses (dashed area) from a two-contract menu. Low-risk types $\left(p \in\left[\frac{c}{d}, \bar{p}\right]\right)$ buy contract $\left(0, c_{A}, 0\right)$ and high-risk types $(p \in[\bar{p}, 1])$ buy contract $\left(0, c_{A}, c / \hat{p}^{*}\right)$. 


\subsection{Contracting under Adverse Selection and Perfect Competition}

Now suppose agents are privately informed about the probability of liability, and the market for insurance is perfectly competitive. There is a perfectly elastic supply of potential insurers capable of freely entering and selling insurance. We follow Rothschild and Stiglitz (1976) in specifying that equilibrium contracts require insurers' profit to be zero, that no entrant could earn a strictly positive profit by offering an alternative contract, and that contracts are exclusive. ${ }^{17}$

We begin the analysis with some useful preliminary results. We first show that the contracting space can be reduced to a single dimension without loss of generality. We then show that the agent's willingness to pay can be re-written in a more compact way, which highlights some analytical properties of this function.

For any contract $\alpha \in \mathcal{A}$, only type $p=p^{*}(\alpha)$ receives perfect insurance. Types $p>p^{*}(\alpha)$ are over-insured and prefer litigation instead of reaching a settlement, which generates losses for the insurer. Types $p<p^{*}(\alpha)$ are under-insured, so they settle, but their bargaining position is not perfectly improved by the insurance policy so the $T P$ extracts bargaining surplus.

Definition 1. Consider $\alpha \in \mathcal{A}$ and $\alpha^{\prime} \in \mathcal{A}$. We say that $\alpha^{\prime}$ dominates $\alpha$ (denoted $\alpha^{\prime} \succeq \alpha$ ) if $W\left(p, \alpha^{\prime}\right)-K\left(p, \alpha^{\prime}\right) \geq W(p, \alpha)-K(p, \alpha)$ for all $p \geq \frac{c}{d}$.

Our next result shows that for each litigation threshold $\rho \in\left[\frac{c}{d}, \infty\right]$ there exists a contract $\alpha^{*}(\rho)$ that dominates all other contracts $\alpha$ that generate the same litigation threshold, i.e., such that $p^{*}(\alpha)=\rho$. This result allows us to reduce the dimensionality of the problem.

Proposition 3 (Undominated Contracts). Let $\alpha^{*}(\rho)=\left(\alpha_{S}^{*}, \alpha_{L}^{*}, \alpha_{D}^{*}\right) \equiv\left(0, c_{A}, \frac{c}{\rho}\right)$ for any $\rho \in\left[\frac{c}{d}, \infty\right]$. Then, $\alpha^{*}(\rho) \succeq \alpha$ for all $\alpha \in \mathcal{A}$ such that $p^{*}(\alpha)=\rho$. Even more, if $\widehat{\alpha} \succeq \alpha$ for all $\alpha \in \mathcal{A}$, with $p^{*}(\widehat{\alpha})=p^{*}(\alpha)=\rho$, then $\widehat{\alpha}=\alpha^{*}(\rho)$.

Proposition 3 shows that for each litigation threshold $\rho \in\left[\frac{c}{d}, \infty\right]$ there is a unique contract $\alpha^{*}$ that maximizes (pointwise) $A$ and $I$ 's joint surplus and satisfies $p^{*}\left(\alpha^{*}\right)=\rho$. In this contract, litigation costs are fully covered, damages are partially covered, and settlements

\footnotetext{
${ }^{17}$ The set of equilibria may expand with non-exclusive contracts (see, e.g., Attar et al., 2011)
} 
are not (directly) covered. By restricting attention to an undominated contract $\alpha^{*}$ it also follows that $p^{* *}\left(\alpha^{*}\right) \leq \frac{c}{d}{ }^{18}$ By using an undominated contract, an insurer improves $A^{\prime} s$ disagreement payoff, enabling $A$ to negotiate a lower settlement fee. Note that contracts that generate a litigation threshold $\rho>1$ are dominated by the contract that generates litigation threshold $\rho=1$. Therefore, we can simply identify undominated contracts with the type that is indifferent between settlement and litigation under that contract. For exposition, we henceforth drop the dependence of $p^{*}(\cdot)$ on $\alpha^{*}$, denoting $p^{*}=p^{*}\left(\alpha^{*}\right)$, and we ignore $p^{* *}\left(\alpha^{*}\right)$. We can then write the willingness to pay and the insurer's expected cost as functions of the agent's type and the threshold type $p^{*}$ induced by any undominated contract:

$$
\begin{aligned}
& W\left(p, p^{*}\right) \equiv W\left(p, \alpha^{*}\left(p^{*}\right)\right)= \begin{cases}(1-\theta)\left[c_{A}+c \frac{p}{p^{*}}\right] & \text { if } p \leq p^{*} \\
{\left[c_{A}+c \frac{p}{p^{*}}\right]-\theta\left(c+c_{A}\right)} & \text { if } p>p^{*}\end{cases} \\
& K\left(p, p^{*}\right) \equiv W\left(p, \alpha^{*}\left(p^{*}\right)\right)= \begin{cases}0 & \text { if } p \leq p^{*} \\
c_{A}+c \frac{p}{p^{*}} & \text { if } p>p^{*}\end{cases}
\end{aligned}
$$

Figure 4 highlights thresholds $p_{1}^{*}$ and $p_{2}^{*}$, with $p_{2}^{*}>p_{1}^{*}$. The contract $\alpha_{1}^{*}$ associated to $p_{1}^{*}$ covers a larger portion of damages than $\alpha_{2}^{*}$ associated to $p_{2}^{*}$. For any $p, W\left(p, p_{1}^{*}\right)>W\left(p, p_{2}^{*}\right)$. Moreover, $W\left(p, p_{1}^{*}\right)-W\left(p, p_{2}^{*}\right)$ is increasing in $p$ and $W\left(p, 1-p^{*}\right)$ is supermodular.

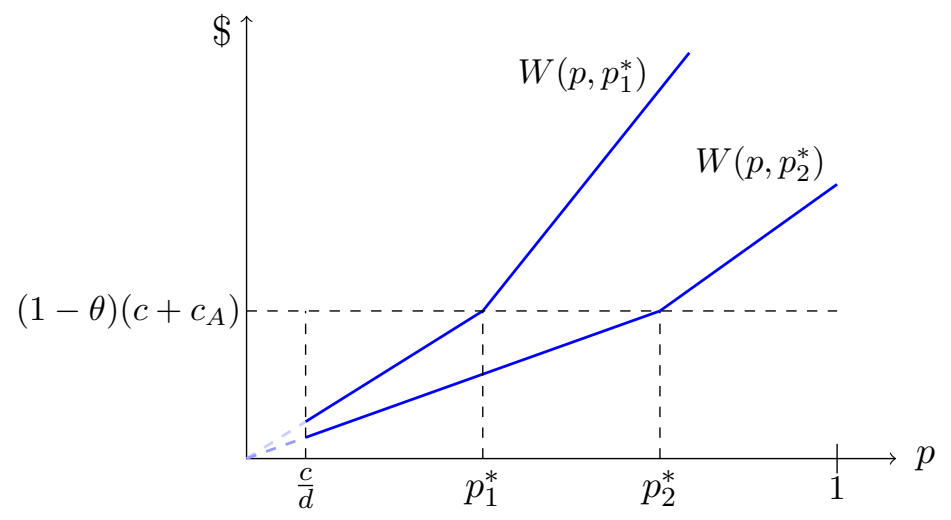

Figure 4: Willingness to pay for two undominated insurance policy contracts, $\alpha_{1}^{*}=\left(0, c_{A}, c / p_{1}^{*}\right)$ and $\alpha_{2}^{*}=\left(0, c_{A}, c / p_{2}^{*}\right)$, indexed by their induced litigation thresholds, $p_{1}^{*}$ and $p_{2}^{*}$, respectively.

\footnotetext{
${ }^{18}$ For any $\alpha \in \mathcal{A}, p^{* *} \leq p^{*}$, and $p^{* *}>\frac{c}{d}$ if and only if $\theta d \alpha_{S}>(1-\theta)\left[\left(d-\alpha_{D}\right) c+\left(c_{A}-\alpha_{L}\right) d\right.$.
} 
The equilibrium price depends on how much litigation is induced by the insurance contracts. If an insurance policy induces all types that buy it to settle, its equilibrium price must be equal to the marginal cost of providing insurance (which we normalize to zero), because the insurer providing the policy bears no additional cost. In contrast, if the insurance induces litigation for some types, the insurer incurs losses on the group of agents who litigate. Hence, to break even, the insurer must earn a strictly positive profit on the other group of agents, so any pooling contract that induces litigation requires cross-subsidization.

Lemma 1. For any distribution of types $F$, a single pooling contract that induces litigation cannot be offered in equilibrium in a perfectly competitive market.

Intuitively, due to the supermodularity of $W\left(p, 1-p^{*}\right)$, a slightly less generous contract could be offered to attract only types that settle (which does not impose any cost on the insurer) and could be sold at a slightly lower, but positive price. This intuition is similar to the cream skimming argument in Rothschild and Stiglitz (1976). Cream-skimming also precludes the possibility of any separating equilibrium.

Lemma 2. For any distribution of types $F$, a separating equilibrium does not exist in a perfectly competitive market.

To separate types in equilibrium, an insurer must sell contracts with different damage coverage at different prices. With common prices, all types would buy the more generous coverage. This rules out two contracts that preclude litigation and are sold for a price of zero. Indeed, to earn zero profit with two contracts that each generate trade, some types must litigate, some types must settle, and the types that settle must pay strictly positive prices (while generating no costs). The reason is that the willingness to pay of types that litigate is below the insurer's cost, so the insurer inevitably loses money on these types. The insurer must therefore earn money from types that settle. But given these requirements, and the supermodularity of $W\left(p, 1-p^{*}\right)$, an alternative insurer can then attract some types that settle, by offering a slightly less generous contract at a slightly lower price. This generates positive profits because all switching types settle. This cream-skimming intuition therefore undermines any such separating equilibrium.

The result in Lemma 2 contrasts with Rothschild and Stiglitz (1976), where a separating 
equilibrium does exist provided there are a sufficiently high number of high-risk types. Also in contrast to Rothschild and Stiglitz (1976), we now show that a simple pooling equilibrium may exist in this market. From Lemma 1 and Lemma 2, the only possible equilibrium is a pooling equilibrium that does not induce litigation.

Theorem 2. If an equilibrium exists, it is a pooling equilibrium with litigation threshold $p_{C, A I}^{*}$ s.t. $F\left(p_{C, A I}^{*}\right)=1$, sold at price zero (marginal cost). An equilibrium exists if and only if

$$
\max _{\hat{p}^{*} \in\left[\frac{c}{d}, p_{C, A I}^{*}\right.}\left\{\frac{(1-\theta) c \cdot\left(p_{C, A I}^{*}-\hat{p}^{*}\right)}{\hat{p}^{*} \cdot p_{C, A I}^{*}} \cdot \max _{\bar{p} \in\left[\frac{c}{d}, \hat{p}^{*}\right]} \bar{p}[1-F(\bar{p})]-\int_{\hat{p}^{*+}}^{p_{C, A I}^{*}}\left[c_{A}+\frac{c p}{\hat{p}^{*}}\right] d F(p)\right\} \leq 0 .
$$

Theorem 2 shows that in a perfectly competitive market for liability insurance, only a pooling equilibrium can exist, and its existence depends on the distribution of types. Intuitively, the condition in Theorem 2 says that a pooling equilibrium exists if the distribution of high-risk types is large enough so that an alternative contract that induces litigation is unprofitable.

Consider a two-types distribution, with $p \in\left\{p_{L}, p_{H}\right\}$ and $\operatorname{Pr}\left(p=p_{H}\right)=\lambda$. The candidate for a pooling equilibrium is a contract that targets $\hat{p}^{*}=p_{H}$, sold to all types at price zero, because no type litigates. The only deviation to consider is contract $\hat{p}^{*}=\bar{p}=p_{L}$. Applying the condition in Theorem 2, this deviation is not profitable if

$$
\lambda \geq \lambda_{A I}^{\text {Pool }} \equiv \frac{(1-\theta) c\left(p_{H}-p_{L}\right) p_{L}}{p_{H}\left(c_{A} p_{L}+c p_{H}\right)}
$$

In other words, when the population has enough $p_{H}$ types, a free contract targeting them is an equilibrium. The $p_{L}$ types also buy this contract, but there is no way to cream skim, because any better contract offered to $p_{L}$ also attracts too many $p_{H}$ types who litigate.

As in the Rothschild and Stiglitz (1976) setting, the equilibrium exists only when the mass of high-risk types is large enough. Under alternative equilibrium concepts, we can also obtain existence when the mass of high-risk types is low. ${ }^{19}$ Finally, note that equilibrium is possible here with a continuum of types, in contrast to first-party insurance, e.g., Riley (1979). ${ }^{20}$

\footnotetext{
${ }^{19}$ In Section B.4 in the Online Appendix, we discuss alternative equilibrium concepts and show that there is an equilibrium under the Wilson anticipatory equilibrium concept (Wilson, 1977).

${ }^{20}$ We provide more details about this point in Section 3.3 (Proposition 5).
} 


\subsection{Contracting under Symmetric Information}

Consider the problem of selling insurance when the insurer and the agent are both uninformed about $p$ but they know its distribution $F{ }^{21}$ In this instance, because every agent is exante identical and there are no externalities among them, there is a single insurance policy offer, which maximizes $A$ and I's joint surplus. By Proposition 3 we restrict attention to undominated contracts. The expected willingness to pay for an undominated contract $\hat{p}^{*}$ is $\mathbf{E}_{p}\left[W\left(p, \hat{p}^{*}\right)\right]$. A monopolist prices this policy at $P_{M}=\mathbf{E}_{p}\left[W\left(p, \hat{p}^{*}\right)\right]$ and extracts all the ex-ante value from the uninformed agents. Hence, the profit maximizing contract for the monopolist targets a type $p_{M, S I}^{*}$ that solves:

$$
\max _{\frac{c}{d} \leq \hat{p}^{*} \leq \infty} \Psi_{S I}\left(\hat{p}^{*}\right) \equiv \mathbf{E}_{p}\left[W\left(p, \hat{p}^{*}\right)-K\left(p, \hat{p}^{*}\right)\right]
$$

In a perfectly competitive market there is free entry, so any active insurer must break even in equilibrium. If insurance contract $\hat{p}^{*}$ is offered in equilibrium its price must be $P_{C}\left(\hat{p}^{*}\right)=$ $\mathbf{E}_{p}\left[K\left(p, \hat{p}^{*}\right)\right]$. Agents buy this contract as long as $\mathbf{E}_{p}\left[W\left(p, \hat{p}^{*}\right)\right] \geq P_{C}\left(\hat{p}^{*}\right)$. Thus, the contract that is offered in equilibrium targets the same type $p_{C, S I}^{*}=p_{M, S I}^{*}=p_{S I}^{*}$, that solves (10). A perfectly competitive market and a monopolist offer the same contract at different prices.

Proposition 4. Let both the agent and the insurer know $F(\cdot)$ but be uninformed about $p$. Then, the liability insurance policy offered by a monopolist or a perfectly competitive market has litigation threshold $p_{M, S I}^{*}=p_{C, S I}^{*}=p_{S I}^{*}$, characterized by the solution to: ${ }^{22}$

$$
\max _{\hat{p} \in\left[\frac{c}{d}, \infty\right]} \Psi_{S I}\left(\hat{p}^{*}\right) \equiv(1-\theta) \int_{c / d}^{\hat{p}^{*}}\left[c_{A}+\frac{c p}{\hat{p}^{*}}\right] d F(p)-\theta\left(c+c_{A}\right)\left[1-F\left(\hat{p}^{*}\right)\right] .
$$

The price of the contract under perfect competition is $P_{C}\left(p_{C, S I}^{*}\right)=\mathbf{E}_{p}\left[K\left(p, p_{S I}^{*}\right)\right]$ and under monopoly is $P_{M}\left(p_{M, S I}^{*}\right)=\mathbf{E}_{p}\left[W\left(p, p_{S I}^{*}\right)\right]$.

Proof. Plug Equation 8 and Equation 9 into Equation 10 take the expectation over $p$.

\footnotetext{
${ }^{21}$ For instance, in the context of defensive patent insurance, a firm and an insurer know that the firm potentially infringes on some patents, but they do not know the scope of the threat, as in facing many potential lawsuits from patent "trolls" or when encountering a patent "thicket."

${ }^{22} \Psi_{S I}(\cdot)$ is upper semi-continuous and decreasing for $\hat{p}^{*}>1$, so a solution must lie in the compact interval $\left[\frac{c}{d}, 1\right]$. This guarantees the existence of a solution.
} 
Equation 11 in Proposition 4 shows that the optimal contract balances out two forces: profiting from types that settle versus incurring losses for types that litigate. Under contract $\hat{p}^{*}$, type $\hat{p}^{*}$ receives perfect insurance, type $p<\hat{p}^{*}$ is under-insured and settles, and type $p>\hat{p}^{*}$ litigates. Types that litigate create a loss for the insurance, because the marginal cost of insurance is positive for them, and exceeds willingness to pay by $\theta\left(c+c_{A}\right)$, which is what the agent would have captured in a settlement negotiation without insurance. Figure 5a illustrates this tradeoff: Area A is the gain in joint surplus from types that settle and corresponds to the term $(1-\theta) \int_{c / d}^{\hat{p}^{*}}\left[c_{A}+\frac{c p}{\hat{p}^{*}}\right] d F(p)$ in equation (11); Area B is the loss in joint surplus from types that litigate and corresponds to the term $-\theta\left(c+c_{A}\right)\left[1-F\left(\hat{p}^{*}\right)\right]$ in equation (11).

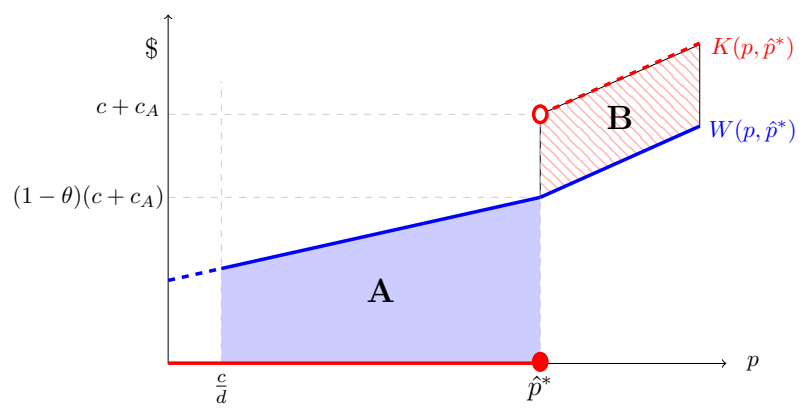

(a) Contract $\hat{p}^{*}$ 's expected joint-surplus gain (solid Area A) and joint-surplus loss (dashed Area B).

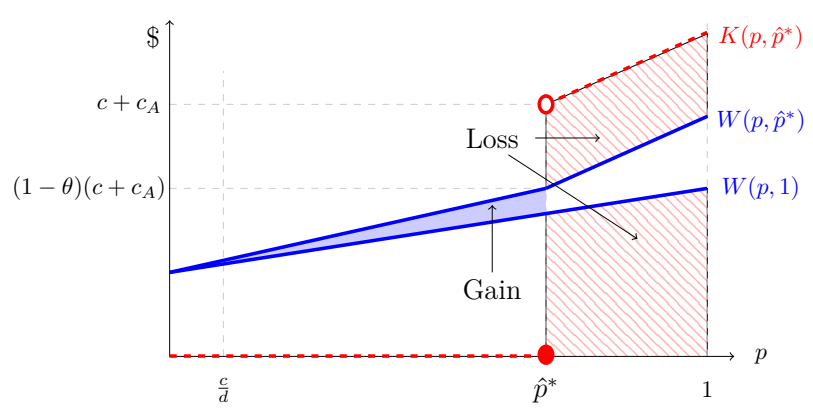

(b) Contract $\hat{p}^{*}$ 's expected joint-surplus gain and loss relative to a contract with $\hat{p}^{*}=1$.

Figure 5: Trade offs when choosing different insurance contracts.

Figure 5b shows the gains and losses of a contract that targets some $\hat{p}^{*}<1$, relative to one that targets $\hat{p}^{*}=1$. The main changes are that the willingness to pay function $W\left(p, \hat{p}^{*}\right)$ is higher (and includes a kink) when $\hat{p}^{*}<1$ is targeted, and types $p>\hat{p}^{*}$ now litigate instead of settling. The gain from this alternative contract comes from making insurance more generous for types that settle in either case - i.e., every type below $\hat{p}^{*}$ is willing to pay more for this contract and generates no costs for the insurer. The losses from this alternative contract come from two sources. First, the cost of providing insurance is larger than the willingness to pay for types above $\hat{p}^{*}$, thus the insurer incurs a net loss for types above $\hat{p}^{*}$. Second, there is an opportunity cost of offering $\hat{p}^{*}<1$ instead of $\hat{p}^{*}=1$. With $\hat{p}^{*}=1$ all types settle and the insurer does not incur costs. The balance, of course, depends on the distribution of types. It is immediate from the figure that if the density of types in a neighborhood of $p=1$ is small enough, then the gain is larger than the loss, and hence we would have an interior solution, $p_{S I}^{*}<1$. 
In Online Appendix B.5 we provide sufficient conditions for uniqueness of the solution to Equation 11 and provide conditions to characterize when the equilibrium threshold $p_{S I}^{*}$ is strictly less than one or equal to one. We find that $p_{S I}^{*}=1$ for distributions that allocate high probability mass to the highest-risk types (see, e.g., Figure 6a) and for any convex $F(p)$. In contrast, $p_{S I}^{*}<1$ obtains for distributions that allocate low probability mass to the highest-risk types (see, e.g., Figure 6b).

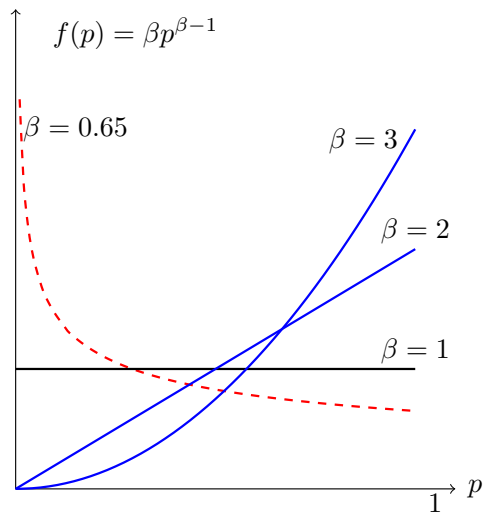

(a) Family $F(p)=p^{\beta}$.

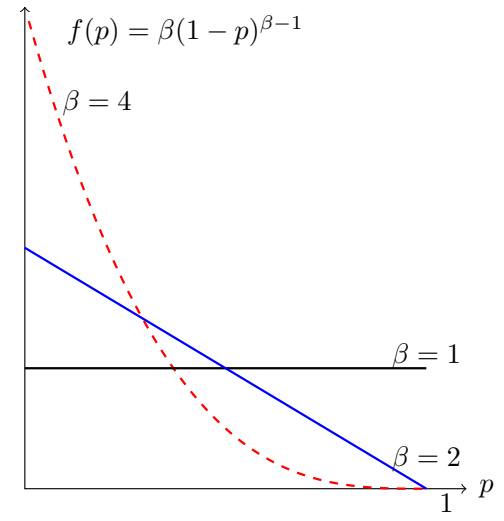

(b) Family $F(p)=1-(1-p)^{\beta}$.

Figure 6: The optimal contract targets the "densest" part of the distribution. When $F(p)=p^{\beta}$, the optimal contract is $p_{S I}^{*}=1$. When $F(p)=1-(1-p)^{\beta}$, the optimal contract is $p_{S I}^{*}<1$.

Consider a two-types distribution, with $p \in\left\{p_{L}, p_{H}\right\}$ and $\operatorname{Pr}\left(p=p_{H}\right)=\lambda$. From Proposition 4 , the optimal contract is either $p_{S I}^{*}=p_{L}$ or $p_{S I}^{*}=p_{H}$. When $\lambda>\frac{(1-\theta) c\left(p_{H}-p_{L}\right)}{p_{H}\left(c+c_{A}\right)+(1-\theta) c\left(p_{H}-p_{L}\right)}$, i.e., when the proportion of high-risk types is relatively large, the optimal contract is $p_{S I}^{*}=$ $p_{H}$. Otherwise, the optimal contract is $p_{S I}^{*}=p_{L}$. That is, the insurer targets the densest part of the distribution with perfect insurance.

The following result shows that whenever the highest-risk type receives perfect insurance under symmetric information, there exists a pooling equilibrium in a competitive market under adverse selection.

Proposition 5. If the optimal contract with symmetric information has $F\left(p_{S I}^{*}\right)=1$, then a pooling equilibrium with $F\left(p_{C, A I}^{*}\right)=1$ exists in the competitive market with adverse selection.

The intuition for Proposition 5 can be seen in Figure 5b. The joint gains from a contract that targets $\hat{p}^{*}<1$ relative to one that targets $\hat{p}^{*}=1$ are higher for a monopoly under symmetric information than for a deviating insurer in a competitive market. This is because 
the monopolist offers only one contract, so the agent's outside option is to not buy liability insurance. In contrast, when a contract with $\hat{p}^{*}=1$ is offered in a competitive market, any deviation must take into account that only types that prefer the deviating contract $\hat{p}^{*}$ over $\hat{p}^{*}=1$ will buy it. Therefore, the gain from deviating from $\hat{p}^{*}=1$ in a competitive market is weakly lower than in the case of monopoly. However, the losses are the same and equal to $\theta\left(c_{A}+c\right)\left[1-F\left(\hat{\hat{p}}^{*}\right)\right]$. Hence, whenever $p_{S I}^{*}=1$ is optimal for a monopolist under symmetric information, no insurer would find it profitable to deviate from a candidate competitive equilibrium contract with $p_{C, A I}^{*}=1$. This result implies, for example, that equilibrium under adverse selection holds for the family of densities in Figure 6a, as well as any convex $F(p)$.

\subsection{Comparison Across Market and Information Structures}

In all environments we have considered, the prospect of litigation shapes equilibrium contracting. However, its effects differ across market and information structures. Under complete information, insurance is set to guarantee settlement and hold the third party to its litigation payoff. In essence, the agent's threat to litigate is maximized, so the third party gets none of the bargaining surplus. Under imperfect information, by contrast, insurance is not perfectly tailored to each agent, and litigation may occur. Generally, litigation is more frequent when information is symmetric.

Proposition 6. In a perfectly competitive market, any equilibrium with symmetric information induces weakly more litigation than any equilibrium with asymmetric information. Under Assumption 1, the monopoly contract with symmetric information induces weakly more litigation than the contract under asymmetric information, i.e., $p_{M, S I}^{*} \leq p_{M, A I}^{*}{ }^{23}$

The result for a perfectly competitive market is simple to understand: Theorem 2 shows that every type settles in an equilibrium under adverse (when it exists); whereas Proposition 4 shows that with symmetric information, the some types may litigate. For the case of a single seller, consider the monopolist's trade-off when choosing $p_{M, A I}^{*}$ in Theorem 1. Figure 7 compares the choice of $p_{M, A I}^{*}=\hat{p}^{*}<1$ versus $p_{M, A I}^{*}=1$. Note that the gain is lower and the losses are higher for a monopolist facing adverse selection, relative to the symmetric

\footnotetext{
${ }^{23}$ This inequality is in the strong set order when the solutions fail to be unique.
} 
information case (Figure 5b). The gain from deviating to $\hat{p}^{*}<1$ is lower under adverse selection because only types above $\bar{p}$ receive damages insurance, and also for all $p>\bar{p}$ we have that $W\left(p, p_{M, A I}^{*}\right)-W(p, 1)>W\left(\bar{p}, p_{M, A I}^{*}\right)-W(\bar{p}, 1)$. The losses from deviating are higher because of the information rents given to types that litigate.

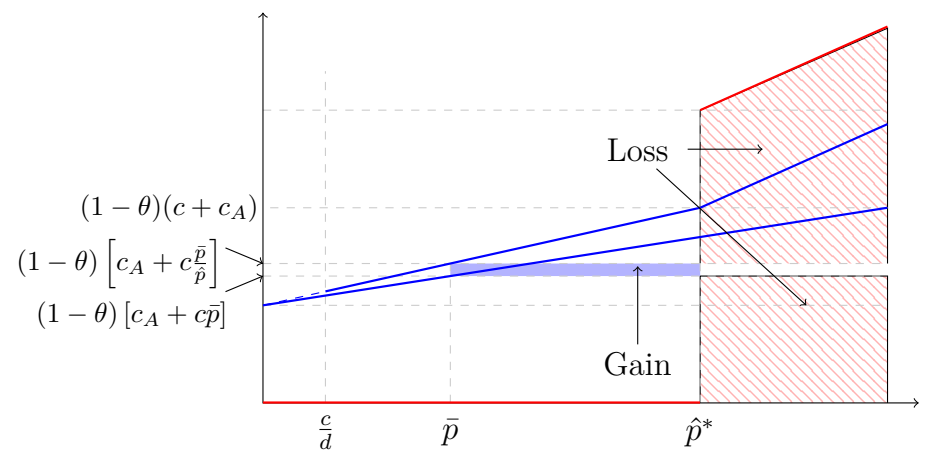

Figure 7: Gain (solid area in blue) and losses (dashed area in red) from offering contract $\hat{p}^{*}<1$ instead of contract $\hat{p}^{*}=1$.

From Proposition 6, the ranking of the equilibrium level of litigation across different informational environments is the same under perfect competition and monopoly. Figure 8 summarizes the results and shows the amount of litigation in equilibrium increases as the insurer and the agent become less informed.

$A$ and $I$ informed $A$ Informed (Adverse Selection) $A$ and $I$ uninformed $\quad$ More litigation

Figure 8: Equilibrium amount of litigation depending on the information structure.

\section{Risk Aversion}

Risk aversion increases the agent's value for risk protection in the case of litigation. Intuitively, the insurer could offer higher damages coverage, which is valuable for risk-averse agents, but in doing so the agent would be more prone to litigate. Under risk neutrality, it is possible to balance the trade-off between improving the agent's bargaining position and inducing litigation, by choosing an appropriate level of damages coverage. Under risk aversion, however, the insurer may need multiple levers to balance these effects out. 
Generally, the case of risk aversion is more technically challenging. First, the agent's wealth may determine the agent's level of risk aversion, which affects bargaining. Second, there is no separability between the agent's cost of insurance and the settlement payoff, in general. So even in the absence of wealth effects, the price of insurance may alter the bargaining outcome. Third, the equilibrium settlement fee $T$, as well as the willingness to pay for insurance, do not generally have closed-form solutions. As a result, the model under risk aversion is not analytically tractable.

We can gain some insights for the case of mean-variance preferences. An agent with these preferences evaluates lottery $X$ according to $U(X)=E(X)-\frac{\sigma \operatorname{Var}(X)}{2}$. Under insurance policy $\alpha=\left(\alpha_{S}, \alpha_{L}, \alpha_{D}\right)$, the certainty equivalent under litigation is

$$
C E(p, \alpha)=w-\left(c_{A}-\alpha_{L}\right)-p\left(d-\alpha_{D}\right)-\frac{\sigma p(1-p)\left(d-\alpha_{D}\right)^{2}}{2}
$$

The only difference with the risk neutral case is the last term, $R P\left(p, \alpha_{D}\right) \equiv \frac{\sigma p(1-p)\left(d-\alpha_{D}\right)^{2}}{2}$. This term corresponds to the agent's risk-premium, which increases both the bargaining surplus and the litigation threshold-type $p^{*}(\alpha)$. The agent's willingness to pay for insurance is non-linear in both $p$ and $\alpha_{D}$, rather than piecewise linear (Proposition 1). Under complete information, however, we show that Proposition 2 still holds - any contract $\alpha$ that maximizes leverage, by setting $p^{*}(\alpha)=p$, is optimal. Under incomplete information, however, contracting becomes more complicated. Proposition 3's conclusion that $\alpha^{*}(p) \equiv\left(0, c_{A}, \frac{c}{p}\right)$ dominates any contract $\alpha^{\prime}$ such that $p^{*}\left(\alpha^{\prime}\right)=p$ no longer holds. The reason is that for fixed litigation threshold $p^{*}$, a lower $\alpha_{D}$ increases $A$ and $I$ 's joint surplus (i.e., $W-K$ ) when $p<p^{*}$ but $\alpha_{D}$ decreases it when $p>p^{*}$. Thus, the multidimensionality of the allocation cannot be reduced. This adds technical challenges to the analysis of competition under adverse selection and to the case of symmetric information.

In Online Appendix Section B.1 we show that the leverage effect remains of primary importance with small risk aversion $\left(\sigma \leq \frac{1}{d}\right)$. The agent's willingness to pay remains monotone in $p$ and supermodular in $p$ and $\alpha_{D}$. Any candidate equilibrium that permits cream-skimming under risk neutrality also permits cream skimming for risk aversion. Thus, the results under perfect competition with adverse selection hold, and the only possible equilibrium pools agents on a no-litigation contract. Under monopoly, the non-linearity of the agent's utility 
function enables the monopolist to sell a more complex incentive-compatible menu of contracts. This also makes the problem analytically intractable. Numerical simulations indicate that, similar to the risk neutral case, low-risk agents buy no damages insurance and high-risk agents pool on a common level of damages coverage. In contrast, though, risk aversion yields a small range of medium-risk types that purchase damages insurance whose level is monotonically increasing in $p$. The case of symmetric uncertainty is also not analytically tractable, but simulations show that for low levels of risk aversion the optimal contract is qualitatively similar to the case of risk neutrality. For larger level of risk aversion, however, the optimal contract under symmetric information features settlement coverage. This shows that in general the leverage effect and risk aversion interact, but for lower levels of risk aversion the leverage effect is the dominant force.

\section{Conclusion}

Third-party liability insurance provides leverage in negotiating a settlement, which creates a distinct source of insurance value that is not present in first-party insurance. This paper is the first to consider this aspect of insurance under adverse selection. We provide a framework in which third-party equilibrium insurance contracts are quite different from first-party insurance contracts, both under monopoly and perfect competition. First, with a monopolist insurer, the optimal contract is qualitatively different from first party insurance studied by Stiglitz (1977) and Chade and Schlee (2012): it may distort types "at the top" (high-risk types) who pursue inefficient litigation, while low-risk types are under-insured. Second, in a perfectly competitive market for third-party insurance only a pooling equilibrium can exist, in contrast to Rothschild and Stiglitz (1976) where only a separating equilibrium can exist. Separating equilibria do not exist in our setting because in such an equilibrium at least one contract would attract both types that settle and types that litigate. Types that settle impose no cost to the insurer and can be "cream skimmed" by offering an alternative contract. A pooling equilibrium, when it exists, delivers imperfect insurance to all but the highest type. The results under monopoly and competition are driven by a discontinuity in the insurer's cost function, which is induced by the agent's choice to settle or to litigate. 
Intuitively, an optimal contract must balance the leverage effect (i.e., improving the agent's bargaining position) and the litigation incentive (i.e., an insured agent is less afraid of litigation). Balancing this trade-off is subtle when the agent holds private information at the time of contracting. Our results suggests that in industries where the leverage effect is a primary motive for buying insurance (e.g., in the patent litigation industry) the insurer should not cover settlements and should use a "litigation cost/damages policy" only. In practice, some companies already offer such insurance, so our results provide an economic rationale for when/why using these policies is optimal. Our model also predicts that coverage for litigation costs is complete, coverage for damages coverage is incomplete and payments for settlement are entirely uncovered. Consistent with this, some existing damages policies include deductibles or "self-insurance retention" clauses, whereby insurance kicks in above a certain level. It would be interesting to study empirically, in policies where insurance covers settlements, how frequently settlement payments occur in excess of deductibles.

In addition to highlighting the negotiating leverage created by liability insurance, we show a potential negative welfare effect of liability insurance: an insured agent could litigate. We show that in both competition and monopoly, equilibria with symmetrically uninformed parties feature more generous coverage and induce more litigation, compared to equilibria where the agent is privately informed about the probability of liability.

Finally, we view our analysis of leverage effects as an important step towards a broader characterization of liability insurance. Our core model abstracts from multiple forces (e.g., risk aversion, bargaining under incomplete information, and alternative control structures) whose influence may vary, in important ways, across types of insurance markets, and elements of liability insurance contracts (e.g. who controls the lawsuit) differ across types of coverage. In our Online Appendix we lay some ground work for studying these forces. We hope our initial insights will stimulate future research.

\section{References}

Allard, Marie, Jean-Paul Cresta, and Jean-Charles Rochet (1997) "Pooling and separating equilibria in insurance markets with adverse selection and distribution costs," The Geneva 
Papers on Risk and Insurance Theory, Vol. 22, pp. 103-120.

Attar, Andrea, Thomas Mariotti, and François Salanié (2011) "Nonexclusive competition in the market for lemons," Econometrica, Vol. 79, pp. 1869-1918.

Azevedo, Eduardo M and Daniel Gottlieb (2017) "Perfect competition in markets with adverse selection," Econometrica, Vol. 85, pp. 67-105.

Buzzacchi, Luigi and Giuseppe Scellato (2008) "Patent litigation insurance and R\&D incentives," International Review of Law and Economics, Vol. 28, pp. 272-286.

Carbajal, Juan Carlos and Jeffrey C Ely (2013) "Mechanism design without revenue equivalence," Journal of Economic Theory, Vol. 148, pp. 104-133.

Chade, Hector and Edward Schlee (2012) "Optimal insurance with adverse selection," Theoretical Economics, Vol. 7, pp. 571-607.

(2019) "Insurance as a Lemons Market: Coverage Denials and Pooling,"Technical report, Working Paper.

Chen, Yongmin and Xinyu Hua (2012) "Ex ante investment, ex post remedies, and product liability," International Economic Review, Vol. 53, pp. 845-866.

Crocker, Keith J and Arthur Snow (1985) "The efficiency of competitive equilibria in insurance markets with asymmetric information," Journal of Public Economics, Vol. 26, pp. $207-219$.

Dana, James D and Kathryn E Spier (1993) "Expertise and contingent fees: The role of asymmetric information in attorney compensation," Journal of Law, Economics, 83 Organization, Vol. 9, pp. 349-367.

Daughety, Andrew and Jennifer Reinganum (2014) "The effect of third-party funding of plaintiffs on settlement," American Economic Review, Vol. 104, pp. 2552-2566.

Duchene, Anne (2015) "Patent Litigation Insurance," Journal of Risk and Insurance.

Farinha Luz, Vitor (2017) "Characterization and uniqueness of equilibrium in competitive insurance," Theoretical Economics, Vol. 12, pp. 1349-1391.

Gravelle, Hugh and Michael Waterson (1993) "No win, no fee: some economics of contingent legal fees," The Economic Journal, Vol. 103, pp. 1205-1220.

Guesnerie, Roger, Pierre Picard, and Patrick Rey (1989) "Adverse selection and moral hazard 
with risk neutral agents," European Economic Review, Vol. 33, pp. 807-823.

Hay, Bruce and Kathryn E Spier (1998) "Settlement of litigation," The New Palgrave Dictionary of Economics and the Law, Vol. 3, pp. 442-451.

Kirstein, Roland (2000) "Risk neutrality and strategic insurance," The Geneva Papers on Risk and Insurance. Issues and Practice, Vol. 25, pp. 251-261.

Kirstein, Roland and Neil Rickman (2004) "" Third Party Contingency" Contracts in Settlement and Litigation," Journal of Institutional and Theoretical Economics JITE, Vol. 160, pp. $555-575$.

Llobet, Gerard and Javier Suarez (2012) "Patent litigation and the role of enforcement insurance," Review of Law and Economics, Vol. 8, pp. 789-821.

Meurer, Michael J (1992) "The gains from faith in an unfaithful agent: Settlement conflicts between defendants and liability insurers," Journal of Law, Economics, Ef Organization, pp. $502-522$.

Miyazaki, Hajime (1977) "The rat race and internal labor markets," The Bell Journal of Economics, pp. 394-418.

Picard, Pierre (1987) "On the design of incentive schemes under moral hazard and adverse selection," Journal of Public Economics, Vol. 33, pp. 305-331.

Ramsay, Colin M, Victor I Oguledo, and Priya Pathak (2013) "Pricing high-risk and lowrisk insurance contracts with incomplete information and production costs," Insurance: Mathematics and Economics, Vol. 52, pp. 606-614.

Reinganum, Jennifer and Louis Wilde (1986) "Settlement, Litigation, and the Allocation of Litigation Costs," The RAND Journal of Economics, Vol. 17, pp. 557-566.

Riley, John G (1979) "Informational equilibrium,” Econometrica, pp. 331-359.

Rothschild, Michael and Joseph Stiglitz (1976) "Equilibrium in Competitive Insurance Markets: An Essay on the Economics of Imperfect Information," The Quarterly Journal of Economics, Vol. 90, pp. 629-649.

Rubinfeld, Daniel L and Suzanne Scotchmer (1993) "Contingent fees for attorneys: An economic analysis," The RAND Journal of Economics, pp. 343-356.

Schwarcz, Daniel and Peter Siegelman (2017) "Law and Economics of Insurance," The Oxford 
Handbook of Law and Economics: Volume 2: Private and Commercial Law, p. 481.

Shavell, Steven (1982) "On liability and insurance," The Bell Journal of Economics, pp. $120-132$.

(2005) "Minimum Asset Requirements and Compulsory Liability Insurance as Solutions to the Judgment-Proof Problem," The RAND Journal of Economics, Vol. 36, pp. 63-77.

Spier, Kathryn E (2007) "Litigation," Handbook of Law and Economics, Vol. 1, pp. 259-342.

Stiglitz, Joseph E (1977) "Monopoly, non-linear pricing and imperfect information: the insurance market," The Review of Economic Studies, pp. 407-430.

Sykes, Alan O (1994) "" Bad Faith" Refusal to Settle by Liability Insurers: Some Implications of the Judgment-Proof Problem," The Journal of Legal Studies, Vol. 23, pp. 77-110.

Townsend, Robert M (1979) "Optimal contracts and competitive markets with costly state verification," Journal of Economic Theory, Vol. 21, pp. 265-293.

Van Velthoven, Ben and Peter van Wijck (2001) "Legal cost insurance and social welfare," Economics Letters, Vol. 72, pp. 387 - 396.

Wilson, Charles (1977) "A model of insurance markets with incomplete information," Journal of Economic Theory, Vol. 16, pp. 167-207.

\section{A Appendix: Proofs}

\section{Proof of Proposition 1}

Proof. For any contract $\alpha \in \mathcal{A}$ we first characterize the endogenous settlement fee $T$. The difficulty is that $T$ depends on the bargaining surplus $S_{B}$, which itself depends on $T$ through the $\min \left\{\alpha_{S}, T\right\}$ term. To organize the exposition of the proof, we have the following result.

Lemma 3. Let $S_{B}^{N B}=\alpha_{S}+c+c_{A}-\alpha_{L}-p \alpha_{D}$ and $T^{N B}=p d-c+(1-\theta) S_{B}^{N B}$.

1. If $S_{B}^{N B}<0$, then $A$ and TP go to litigation. 
2. If $S_{B}^{N B} \geq 0$, then $A$ and $T P$ settle and $T P$ receives a transfer $T$ given by

$$
T= \begin{cases}\alpha_{S} & \text {, if } T^{N B} \leq \alpha_{S}, \\ T^{N B} & \text {, if } T^{N B} \geq \alpha_{S} .\end{cases}
$$

Proof. We consider two cases. First, suppose $\alpha_{S} \leq p d-c$. TP's outside option is $p d-c$, so the Nash bargaining transfer must satisfy $T \geq p d-c$ for any $\theta$. Hence $T \geq \alpha_{S}$ and $S_{B}=S_{B}^{N B}$. In this case, the Nash bargaining transfer is $T=T^{N B}$.

Second, suppose $\alpha_{S}>p d-c$. In this case, any transfer $T \in\left[p d-c, \alpha_{S}\right]$ gives $A$ a payoff of 0 and $T P$ a payoff of $u_{T P}=T$. Thus, settlement agreements with $T<\alpha_{S}$ are Pareto dominated by $T=\alpha_{S}$, so the Nash bargaining solution must have $T \geq \alpha_{S}$. We can now maximize the Nash bargaining product subject to this additional constraint. When $\theta$ is sufficiently large, we get a corner solution. In particular, there exists $\bar{\theta}$ that solves

$$
p d-c+(1-\theta)\left[\alpha_{S}+c+c_{A}-\alpha_{L}-p \alpha_{D}\right]=\alpha_{S},
$$

and for any $\theta>\bar{\theta}$ we have $T=\alpha_{S}$, while for $\theta<\bar{\theta}$ we have $T=T^{N B}$. Finally, note that we can re-write

$$
\bar{\theta}=\frac{p d-c-\alpha_{S}+S_{B}}{S_{B}}=\frac{p\left(d-\alpha_{D}\right)+c_{A}-\alpha_{L}}{\alpha_{S}+c+c_{A}-\alpha_{L}-p \alpha_{D}} .
$$

To explain the result in Lemma 3, consider the frontier of A and TP's bargaining set, for any $\alpha$, illustrated in Figure 9. When $\alpha_{S} \leq p d-c$, shown in Figure 9(a), the settlement transfer is larger than TP's outside so $T \geq p d-c \geq \alpha_{S}$. The solution is the standard Nash bargaining solution, so $S_{B}=S_{B}^{N B}, T=T^{N B}$ and the agent's payoff is $-T+\alpha_{S} \leq 0$.

When $\alpha_{S}>p d-c$, shown in Figure 9(b), the agent's payoff is constant and equal to zero whenever $T \leq \alpha_{S}$, which corresponds to the horizontal segment in Figure 9(b). Any settlement agreement with $T<\alpha_{S}$ is Pareto dominated by one where $T=\alpha_{S}$, so any Pareto efficient solution has $T \geq \alpha_{S}$. When $T^{N B} \leq \alpha_{S}$, the Nash bargaining solution is exactly at the kink, $\left(\alpha_{S}, 0\right)$. Otherwise, the Nash bargaining solution is on the interior of the downward sloping region of the frontier, and $T=T^{N B}$. 


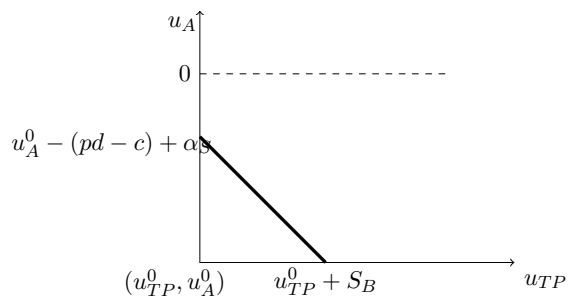

(a) $\alpha_{S} \leq p d-c$.

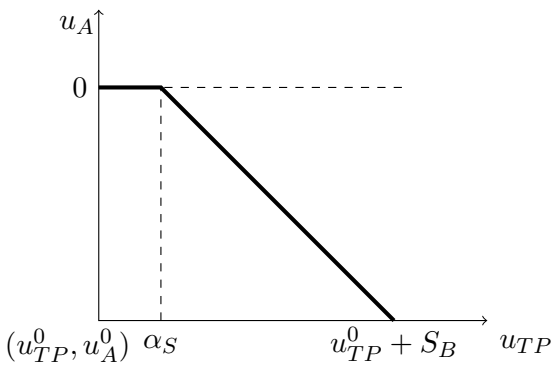

(b) $\alpha_{S}>p d-c$.

Figure 9: Frontier of the bargaining set for different values of $\alpha_{S}$, where the disagreement payoffs are at the origin, with $u_{T P}^{0}=p d-c$ and $u_{A}^{0}=V_{L}(p, \alpha)$.

Lemma 3 implies that litigation occurs for agents of type $p$ larger than

$$
p^{*} \equiv \frac{\alpha_{S}+c+c_{A}-\alpha_{L}}{\alpha_{D}}
$$

and when $p \leq p^{*}$ there is settlement and the agent's settlement payoff is:

$$
V_{S}(p, \alpha)=-\max \left\{0, T^{N B}-\alpha_{S}\right\}=\min \left\{0, \alpha_{S}-T^{N B}\right\}
$$

The agent's net payoff from settling is negative when $T>\alpha_{S}$, which occurs when the Nash bargaining solution is on the downward-sloping part of the frontier. Otherwise, when the Nash bargaining solution is at the kink, insurance fully covers the settlement fee and the agent's payoff is 0 . Equation (13) defines a threshold type $p^{* *}$ such that for any type $p \leq p^{* *}$ the agent's settlement payoff is zero, where

$$
p^{* *} \equiv \frac{\theta\left(\alpha_{S}+c\right)-(1-\theta)\left(c_{A}-\alpha_{L}\right)}{d-\alpha_{D}(1-\theta)} .
$$

Consider an insurance policy $\alpha=\left(\alpha_{S}, \alpha_{L}, \alpha_{D}\right) \in \mathcal{A}$ that generates thresholds $p^{*}$ and $p^{* *}$ as defined in equations (12) and (14). The payoff of settlement for an agent of type $p$ covered by insurance policy $\alpha$ is $V_{S}(p, \alpha)=0$ when $p \in\left[\frac{c}{d}, p^{* *}\right]$ and $V_{S}(p, \alpha)=\theta\left[\alpha_{s}-(p d-c)\right]-$ $(1-\theta)\left[p\left(d-\alpha_{D}\right)+\left(c_{A}-\alpha_{L}\right)\right] p \in\left(\max \left\{\frac{c}{d}, p^{* *}\right\}, p^{*}\right]$; whereas the payoff of litigation is $V_{L}(p, \alpha)=-c_{A}-p d+\alpha_{L}+p \alpha_{D}$. Without insurance $(\alpha=0)$ the agent settles and gets a payoff of $V_{S}(p, 0) \equiv-p d-c_{A}+\theta\left(c+c_{A}\right)$. The willingness to pay for insurance is then the difference in the agent's payoff with and without insurance, which is given by Equation (2). It's easy to see that the expected cost of the insurer is given by Equation (3). 


\section{Lemma 4}

Lemma 4. For any IC mechanism, $\left\{p: p=p^{*}(\alpha(p))\right\}$ has measure zero.

Proof. We proceed in two steps: (i) we show that IC implies that within a particular region of nearby types, at most a finite set of types can receive perfect insurance; (ii) we show that the type space consists of finitely many such regions. As a consequence, only finitely many types can receive perfect insurance in any IC mechanism.

Consider an IC mechanism and suppose two types $p_{1}$ and $p_{2}$ receive contracts $\alpha^{1} \equiv \alpha\left(p_{1}\right)$ and $\alpha^{2} \equiv \alpha\left(p_{2}\right)$ such that $p_{1}=p^{*}\left(\alpha^{1}\right)$ and $p_{2}=p^{*}\left(\alpha^{2}\right)$, i.e. $p_{1}$ and $p_{2}$ both receive perfect insurance. WLOG, suppose $p_{1}<p_{2}$.

Step 1: Suppose $p_{1} \geq p^{* *}\left(\alpha^{2}\right)$. IC requires: ${ }^{24}$

$$
\begin{aligned}
& W\left(p_{1}, \alpha^{1}\right)-T\left(p_{1}\right) \geq W\left(p_{1}, \alpha^{2}\right)-T\left(p_{2}\right), \\
& W\left(p_{2}, \alpha^{2}\right)-T\left(p_{2}\right) \geq W\left(p_{2}, \alpha^{1}\right)-T\left(p_{1}\right) .
\end{aligned}
$$

Adding up these conditions we have $W\left(p_{2}, \alpha^{2}\right)-W\left(p_{1}, \alpha^{2}\right) \geq W\left(p_{2}, \alpha^{1}\right)-W\left(p_{1}, \alpha^{1}\right)$. Given that $p_{2}>p_{1}$, if type $p_{2}$ reports $p_{1}$ and receives the contract $\alpha^{1}$ with litigation threshold $p_{1}$, then it would litigate. Using Equation 2, we have: $W\left(p_{2}, \alpha^{1}\right)-W\left(p_{1}, \alpha^{1}\right)=\alpha_{D}^{1}\left(p_{2}-p_{1}\right)$. On the other hand, if type $p_{1}$ reports $p_{2}$ and receives the contract $\alpha^{2}$ with litigation threshold $p_{2}$, then it would settle. Since $p_{1} \geq p^{* *}\left(\alpha^{2}\right)$, this would yield partial settlement coverage for $p_{1}$. Using Equation 2, we therefore have $W\left(p_{2}, \alpha_{2}\right)-W\left(p_{1}, \alpha_{2}\right)=\alpha_{D}^{2}\left(p_{2}-p_{1}\right)(1-\theta)$. With these expressions above, we can write $\alpha_{D}^{2}(1-\theta) \geq \alpha_{D}^{1}$. Therefore, IC implies that if both $p_{1}$ and $p_{2}$ get perfect insurance, the higher type must get higher damages coverage in an IC mechanism. Now suppose, for the sake of a contradiction, that there is an infinite set of types $p_{1}<p_{2}<\ldots p_{n}<\ldots$, with $p_{i} \geq p^{* *}\left(p_{i+1}\right)$ for all $i$. Then the above argument implies that $\alpha_{D}^{n-1}(1-\theta)^{n} \geq \alpha_{D}^{1}, \quad$ for all $n \geq 2$. Given that $\theta>0$, we have $(1-\theta)^{n} \rightarrow 0$, so there exists $k \geq 2$ large enough such that $\alpha_{D}^{k}>d$. This contradicts the assumption $\alpha_{D} \leq d$. Therefore IC implies that for any type $p$ who receives perfect insurance, i.e. $p=p^{*}(\alpha(p))$, there are at most finitely many types above $p^{* *}(\alpha(p))$ who also receive perfect insurance.

\footnotetext{
${ }^{24}$ To simplify notation, we index contracts by the litigation thresholds that they generate, i.e. $p_{1}$ and $p_{2}$.
} 
Step 2: Now suppose, for the sake of a contradiction, that there are infinitely many regions of the type space within which at least one type gets perfect insurance. That is, there are infinitely many types $p_{1}<p_{2}<\ldots<p_{n}<\ldots$, with $p_{i}<p^{* *}\left(\alpha\left(p_{i+1}\right)\right)$, such that $p_{i}=p^{*}\left(\alpha\left(p_{i}\right)\right)$.

Consider the region $\left[p^{* *}(\alpha(p)), p^{*}(\alpha(p))\right]$ for any type $p$. Using the definitions of $p^{* *}$ and $p^{*}$,

$$
p^{*}(\alpha(p))-p^{* *}(\alpha(p))=\frac{\left(\alpha_{S}+c\right)\left(d-\alpha_{D}\right)+d\left(c_{A}-\alpha_{L}\right)}{\alpha_{D}\left(d-\alpha_{D}(1-\theta)\right)} .
$$

If $d-\alpha_{D} \geq m_{D}>0$ or $c_{A}-\alpha_{L} \geq m_{L}>0$ for infinitely many types, then $p_{i+1}-p_{i}>M$ for an infinite number of types, for some uniform bound $M>0$. Therefore we have infinitely many disjoint intervals $\left[p_{i}, p_{i+1}\right]$ in $[0,1]$ with length of at least $M$, which is a contradiction.

Hence we have at most finitely many regions of the form $\left[p^{* *}(\alpha(p)), p^{*}(\alpha(p))\right]$ where type $p$ gets perfect insurance, and within each region there are at most finitely many types who receive perfect insurance, from step 1. Thus the set of types at the kink, $\left\{p: p=p^{*}(\alpha(p))\right\}$, who receive perfect insurance, has measure zero.

\section{Lemma 5}

Lemma 5. Incentive compatibility requires $\alpha_{D}(p)$ to be non-decreasing.

Proof. For any $p, \tilde{p}$ incentive compatibility requires:

$$
W(p, \alpha(p))-T(p) \geq W(p, \alpha(\tilde{p}))-T(\tilde{p})
$$

Let $\Delta(p, \tilde{p})=W(p, \alpha(p))-T(p)-[W(p, \alpha(\tilde{p}))-T(\tilde{p})]$. We have

$$
\Delta(p, \tilde{p})=\int_{\tilde{p}}^{p}\left[\frac{\partial W(s, \alpha(s))}{\partial p}-\frac{\partial W(s, \alpha(\tilde{p}))}{\partial p}\right] d s
$$

Therefore, $\Delta(p, \tilde{p}) \geq 0 \Leftrightarrow \int_{\tilde{p}}^{p}\left[\frac{\partial W(s, \alpha(s))}{\partial p}-\frac{\partial W(s, \alpha(\tilde{p}))}{\partial p}\right] d s \geq 0$. A more compact form of the same IC condition is

$$
\int_{\tilde{p}}^{p}\left[\frac{\partial W(s, \alpha(s))}{\partial p}-\frac{\partial W(s, \alpha(\tilde{p}))}{\partial p}\right] d s \geq 0 \Leftrightarrow \int_{\tilde{p}}^{p}\left[\int_{\tilde{p}}^{s} \frac{d}{d t}\left[\frac{\partial W(s, \alpha(t))}{\partial p}\right]_{t=u} d u\right] d s \geq 0 .
$$


The term inside the integral must be weakly positive, because the inequality must hold for any $p, p^{\prime}$. When $\alpha(t)=\left(\alpha_{S}(t), \alpha_{L}(t), \alpha_{D}(t)\right)$, we have $\frac{d}{d t}\left[\frac{\partial W(s, \alpha(t))}{\partial p}\right]=\sum_{i} \frac{\partial W(s, \alpha(t))}{\partial p \partial \alpha_{i}} \frac{d \alpha_{i}(t)}{d t}$. Therefore IC implies

$\sum_{i=1}^{N} \frac{\partial W(s, \alpha(t))}{\partial p \partial \alpha_{i}} \frac{d \alpha_{i}(t)}{d t}=\frac{\partial W(s, \alpha(t))}{\partial p \partial \alpha_{S}} \frac{d \alpha_{S}(t)}{d t}+\frac{\partial W(s, \alpha(t))}{\partial p \partial \alpha_{L}} \frac{d \alpha_{L}(t)}{d t}+\frac{\partial W(s, \alpha(t))}{\partial p \partial \alpha_{D}} \frac{d \alpha_{D}(t)}{d t} \geq 0$

$\forall s, t$. By Equation 2, we have $\frac{\partial W(s, \alpha(t))}{\partial p \partial \alpha_{S}}=0$ and $\frac{\partial W(s, \alpha(t))}{\partial p \partial \alpha_{L}}=0$, so the condition above simplifies to $\frac{\partial W(s, \alpha(t))}{\partial p \partial \alpha_{D}} \frac{d \alpha_{D}(t)}{d t} \geq 0, \quad \forall s, t$. Since $\frac{\partial W(s, \alpha(t))}{\partial p \partial \alpha_{D}} \geq 0$, this implies $\alpha_{D}(p)$ is increasing.

\section{Proof of Theorem 1}

Proof. The insurer's objective can be written as $\max _{\alpha} \int G(p, \alpha) d F(p)$, subject to $\alpha_{S} \geq 0, \alpha_{L} \in$ $\left[0, c_{A}\right]$ and $\alpha_{D} \in[0, d]$, and where

$$
G(p, \alpha)=W(p, \alpha)-K(p, \alpha)-\frac{\partial W(p, \alpha)}{\partial p}\left(\frac{1-F(p)}{f(p)}\right)
$$

and $h(p) \equiv \frac{1-F(p)}{f(p)}$ is the inverse hazard rate.

Rather than solving this problem directly, we fix $p \in[c / d, 1]$ and we look for a contract that maximizes $G(p, \alpha)$ pointwise. Any such $\alpha$ must be such that $p \in\left(p^{* *}(\alpha), p^{*}(\alpha)\right]$, because $G(p, \alpha)$ is increasing in $p$ for $p \leq p^{*}(\alpha)$ and negative above $p^{*}(\alpha)$. Then we have

$$
\begin{array}{r}
\theta \alpha_{S}-(p d-c)<(1-\theta)\left[c+c_{A}-\alpha_{L}-p \alpha_{D}\right] \\
p \alpha_{D} \leq \alpha_{S}+c+c_{A}-\alpha_{L}
\end{array}
$$

So the point-wise maximization reduces to maximizing $G(p, \alpha)$ subject to (15) and (16). We solve this problem in two steps.

Step 1: Define $\bar{p}$ as the solution to $p=h(p)$, which exists under Assumption 1.

When $p \leq \bar{p}$, the insurer wants to set $\alpha_{D}(p)=0, \alpha_{L}$ as large as possible, and $\alpha_{S}$ as small as possible, in the region where (15) and (16) hold. It is easy so see that contract $\alpha(p)=\left(0, c_{A}, 0\right)$ 
satisfies all of these objectives. Thus, this is the optimal menu in the region $p \leq \bar{p}$.

Step 2: When $p>\bar{p}$, we solve the following linear optimization problem

$$
\max _{\alpha_{S}, \alpha_{L}, \alpha_{D}} \alpha_{L}-\alpha_{S}+(p-h(p)) \alpha_{D}
$$

subject to the linear constraints (15) and (16). In general, the solution to this problem is contract $\alpha=\left(0, c_{A}, c / p\right)$, where (16) binds and (15) does not bind.

The problem with this unconstrained solution is that $\alpha_{D}(p)=\frac{c}{p}$ violates the monotonicity constraint from Lemma 5. Thus we need to use ironing to obtain the constrained solution. In our environment this is simple: the unconstrained solution is one where $\alpha_{D}$ is 0 up to $\bar{p}$ and strictly decreasing above $\bar{p}$. The ironed solution requires $\alpha_{D}(p)$ to be constant for $p>\bar{p}$. For any $\alpha_{D}$, it follows that type $\hat{p}^{*}=\frac{c}{\alpha_{D}}$ is indifferent between settling and litigating. We can then substitute in $\alpha_{S}=0, \alpha_{L}=c_{A}$ and $\alpha_{D}=\frac{c}{\hat{p}^{*}}$ into the objective function, and find the optimal threshold type, $p_{M, A I}^{*}$, that solves the problem in the statement of the theorem.

\section{Proof of Proposition 3}

Proof. Fix $\rho \in\left[\frac{c}{d}, \infty\right]$. First, consider a contract $\alpha=\left(\alpha_{S}, \alpha_{L}, \alpha_{D}\right)$ such that $p^{*}(\alpha)=\rho$ and $p^{* *}(\alpha)>\frac{c}{d}$. Then, we must have $\alpha_{S}>0$, because $p^{* *}>\frac{c}{d}$ if an only if $\theta d \alpha_{S}>$ $(1-\theta)\left[\left(d-\alpha_{D}\right) c+\left(c_{A}-\alpha_{L}\right) d\right]$. It is possible to find an alternative contract $\alpha^{\prime}=\left(\alpha_{S}^{\prime}, \alpha_{L}^{\prime}, \alpha_{D}^{\prime}\right)$, with $\alpha_{S}^{\prime}=0$ and $\alpha_{D}^{\prime} \leq \alpha_{D}$, such that $p^{*}\left(\alpha^{\prime}\right)=\rho$ and $p^{* *}\left(\alpha^{\prime}\right)<\frac{c}{d}$. From Equation (4) it is easy to see that: (a) $W(p, \alpha)-K(p, \alpha)<W\left(p, \alpha^{\prime}\right)-K\left(p, \alpha^{\prime}\right)$ for $p \in\left[\frac{c}{d}, p^{* *}(\alpha)\right]$; (b) $W(p, \alpha)-K(p, \alpha) \leq W\left(p, \alpha^{\prime}\right)-K\left(p, \alpha^{\prime}\right)$ for $p \in\left[p^{* *}(\alpha), \rho\right]$; and (c) $W(p, \alpha)-K(p, \alpha)=$ $W\left(p, \alpha^{\prime}\right)-K\left(p, \alpha^{\prime}\right)$ for $p \in[\rho, 1]$. Then $\alpha^{\prime} \succeq \alpha$.

Next, consider a contract with $\alpha_{D}>0, p^{*}(\alpha)=\rho$ and $p^{* *}(\alpha) \leq \frac{c}{d}$, so the first region in Equation 4 disappears and $\alpha_{S}$ influences $W-K$ only through $p^{*}(\alpha)$. When $\theta<1$ and $p \neq \rho$, $\alpha_{D}$ multiplies the negative term when $p<p^{*}$, so it is weakly dominant to set $\alpha_{D}$ as small as possible. This can be accomplished by finding a contract $\alpha$ that minimizes $\alpha_{D}=\frac{\alpha_{S}+c+c_{A}-\alpha_{L}}{\rho}$ subject to $p^{* *}(\alpha) \leq \frac{c}{d} \Leftrightarrow \alpha_{S} \leq \frac{\theta\left(c+c_{A}-\alpha_{L}\right)}{\theta c+(1-\theta) \rho}\left(\rho-\frac{c}{d}\right)$. The solution to this problem is to set $\alpha_{S}^{*}=c_{A}-\alpha_{L}^{*}=0$, and $\alpha_{D}^{*}=\frac{c}{\rho}$. 
Next, consider a contract with $\alpha_{D}=0$ (or the case where $\theta=1$ ). Then, it is clear that a contract with $\alpha_{S}>0$ is dominated by one with $\alpha_{S}=0$.

Finally, suppose that $\alpha^{\prime} \succeq \alpha$ for all $\alpha \in \mathcal{A}$ with $p^{*}(\alpha)=\bar{p}$. We must have $p^{* *}\left(\alpha^{\prime}\right)<\frac{c}{d}$. Because $\alpha^{\prime} \succeq \alpha^{*}$ and $\alpha^{*} \succeq \alpha^{\prime}$, we must have $\alpha_{D}^{\prime}=\alpha_{D}^{*}$. But given that $\alpha_{S}^{*}=0$ and $\alpha_{L}^{*}=c_{A}$, we have $\alpha_{D}^{*} p^{*}=c$. This implies that $c=\alpha_{S}^{\prime}+c+c_{A}-\alpha_{L}^{\prime}$, implying $\alpha_{S}^{\prime}=c_{A}-\alpha_{L}^{\prime}=0$.

\section{Proof of Lemma 1}

Proof. Consider a distribution of types $F \sim[0,1]$, and an insurance contract $\alpha$ with associated litigation threshold $p^{*}(\alpha)$. Suppose for a contradiction that $p^{*}$ is offered in equilibrium at price $P$ and $F\left(p^{*}\right)<1$. Since $F\left(p^{*}\right)<1$, then there is a positive mass of types that litigate, for which the insurer incur losses. To break even in equilibrium, it must be that price $P>0$. Consider an alternative contract $\hat{p}^{*}=p^{*}+\varepsilon$ sold at price $\hat{P}<P$, with $\varepsilon$ sufficiently small. This new contract offers a lower damages coverage, is cheaper, and preferred by types $p<\hat{p}^{*}$ over contract $p^{*}$ and not preferred for types $p>\hat{p}^{*}$ as long as $W\left(p, p^{*}\right)-P<W\left(p, \hat{p}^{*}\right)-\hat{P}$, for all $p<\hat{p}^{*}$ and $W\left(p, p^{*}\right)-P>W\left(p, \hat{p}^{*}\right)-\hat{P}$, for all $p>\hat{p}^{*}$. These conditions are satisfied for $\hat{P}=P+W\left(\hat{p}^{*}, \hat{p}^{*}\right)-W\left(\hat{p}^{*}, p^{*}\right)=P-\frac{c}{p^{*}} \varepsilon$, which is positive for $\varepsilon$ small enough. Thus, contract $\hat{p}^{*}$ only attracts types that settle and it is sold at a positive price. Hence, there is a profitable deviation, which is a contradiction.

\section{Proof of Lemma 2}

Proof. For a contradiction let $\mathcal{M}$ be the set of contracts offered in equilibrium. In a separating equilibrium, at least two of these contracts must attract a different set of types. Let $p_{1}^{*}$ and $p_{2}^{*}$ with $p_{1}^{*}<p_{2}^{*}$, sold at prices $P_{1}$ and $P_{2}$, respectively, be such a pair of contracts. Let $D_{i} \subseteq[0,1]$ the set of types that prefer contract $p_{i}^{*}$,

$$
D_{i}=\left\{p \in\left[\frac{c}{d}, 1\right]: W\left(p, p_{i}^{*}\right)-P_{i} \geq W\left(p, p_{j}^{*}\right)-P_{j}, \quad \text { for all } p_{j}^{*} \in \mathcal{M}\right\}
$$

Let $D_{i}(S)=D_{i} \cap\left[0, p_{i}^{*}\right]$ and $D_{i}(L)=D_{i} \cap\left(p_{i}^{*}, 1\right]$ be the set of types that buy contract $p_{i}^{*}$ and that settle and litigate, respectively. If the measure of the set $D_{i}(L)$ is zero, then $P_{i}=0$, 
since the insurer would not bear any costs by offering $p_{i}^{*}$. But it cannot be that $D_{1}(L)$ and $D_{2}(L)$ have both measure zero, since they would be sold at price zero types would pool at $p_{1}^{*}$ (see Figure 4). This rules out separating equilibrium with any pair of contracts such that litigation is precluded under both, because such a pair would need to be priced at zero in equilibrium and types would pool at the lowest $p_{i}^{*}$. So, in any separating equilibrium we must have a positive measure of $D_{i}(L)>0$ for some $i \in\{1,2\}$. Without loss of generality, suppose that $\mu_{F}\left(D_{1}(L)\right)>0$. Notice that if $\mu_{F}\left(D_{1}(S)\right)=0$, insurers incur in losses by selling this contract. Thus, contract $p_{1}^{*}$ must attract types that settle an must sell at a positive price $P_{1}>0$. We can construct a new contract $\hat{p}^{*}=p_{1}^{*}+\varepsilon$ sold at price $\hat{P}>0$ that is a profitable deviation from $p_{1}^{*}$. This implies that $p_{1}^{*}$ cannot be offered in equilibrium, because then $p_{1}^{*}$ would attract only types that litigate and lose money. This is a contradiction.

\section{Proof of Theorem 2}

Proof. By Proposition 1, there is no pooling equilibrium at $p_{C, A I}^{*}$ such that $F\left(p_{C, A I}^{*}\right)<1$. Hence, the only candidate is $p_{C, A I}^{*}$ such that $F\left(p_{C, A I}^{*}\right)=1$.

To show existence of equilibrium, we first consider possible deviations whereby an insurer offers a menu of contracts $M$ that competes against the contract with $p_{C, A I}^{*}$. First, notice that $M$ cannot contain any contracts that target types above $p_{C, A I}^{*}$, as these would be dominated by the $p_{C, A I}^{*}$ contract. Moreover, any contract in $M$ that targets a type $\hat{p}^{*}$ must have $W\left(p, \hat{p}^{*}\right)>W\left(p, p_{C, A I}^{*}\right)$. Furthermore, $W\left(p, \hat{p}^{*}\right)-W\left(p, p_{C, A I}^{*}\right)$ is continuous, increasing, piecewise linear, with a kink at $p=\hat{p}^{*}$, and is supermodular, i.e. $W\left(p, \hat{p}^{*}\right)-W\left(p, p_{C, A I}^{*}\right)$ has the same features as $W\left(p, p^{*}\right)$ from Equation 8 and Figure 4; specifically:

$$
W\left(p, \hat{p}^{*}\right)-W\left(p, p_{C, A I}^{*}\right)= \begin{cases}(1-\theta)\left[c p \frac{p_{A I}^{C}-\hat{p}^{*}}{p_{C, A I}^{*} \hat{p}^{*}}\right] & \text { if } p \leq \hat{p}^{*} \\ c \frac{p}{\hat{p}^{*}}-\theta c-(1-\theta) c \frac{p}{p_{C, A I}^{*}} & \text { if } p>\hat{p}^{*}\end{cases}
$$

From the proof of Theorem 1, given this willingness-to-pay function, the optimal menu $M$ consists of at most 2 contracts. Here we have that $W\left(0, \hat{p}^{*}\right)-W(0,1)=0$, which means that the optimal deviation menu $M$ in fact consists of a single contract, which targets some type $\hat{p}^{*}$. I.e. the optimal deviation menu given a candidate contract $W\left(p, p_{A I}^{C}\right)$ is simpler than 
the optimal menu in the absence of such a candidate, because it excludes types below some cutoff, and only features a single contract targeted at $\hat{p}^{*}$. Therefore we can restrict attention to deviations $M$ consisting of a single contract.

Now consider a single deviation contract with $\hat{p}^{*}$, sold at price $\hat{P}$. It is a profitable deviation if it attracts enough low-risk types that settle, to compensate the losses from high-risk types above $\hat{p}^{*}$ that litigate. Let $\bar{p}$ be the (unique by single crossing) type that is indifferent between $\hat{p}^{*}$ at price $\hat{P}$ and $p_{C, A I}^{*}$ for free. Then,

$$
W\left(\bar{p}, p_{C, A I}^{*}\right)=W\left(\bar{p}, \hat{p}^{*}\right)-\hat{P} \Rightarrow \hat{P}=\bar{p}\left[\frac{(1-\theta) c \cdot\left(p_{C, A I}^{*}-\hat{p}^{*}\right)}{\hat{p}^{*} p_{C, A I}^{*}}\right]
$$

Next, we only consider contracts such that $\hat{p}^{*}>\bar{p}$. In any other case, the insurer loses money by offering the deviation. Then, the profit of contract $\hat{p}^{*}$ at price $\hat{P}$ is given by

$$
\hat{P}[1-F(\bar{p})]-\int_{\hat{p}^{*}}^{1} K\left(p, \hat{p}^{*}\right) d F(p)=\hat{P}[1-F(\bar{p})]-\int_{\hat{p}^{*}}^{1}\left[c_{A}+\frac{c p}{\hat{p}^{*}}\right] d F(p)
$$

We can choose the best cutoff point $\bar{p}$ for a given $\hat{p}^{*}$ and then choose the best deviation. Hence, there is no profitable deviation when the condition in the Theorem holds.

\section{Proof of Proposition 5}

Proof. Without loss of generality, suppose that $F\left(p^{*}\right)=1$ implies that $p^{*}=1$. If $p^{*}=1$ is optimal under symmetric information, then for any $\widetilde{p} \in\left(\frac{c}{d}, 1\right)$, we have

$$
\int_{\frac{c}{d}}^{\widetilde{p}}(1-\theta) c p\left(\frac{1-\widetilde{p}}{\widetilde{p}}\right) d F(p)-\int_{\widetilde{p}}^{1}\left\{c_{A}+c[\theta+(1-\theta) p]\right\} d F(p)<0
$$

To establish that a pooling equilibrium exists with $p^{*}=1$ under competition, we need to show that there are no $\widetilde{p}$ and $\bar{p}$ such that alternative insurance $\widetilde{p}$ sold for price $\widetilde{P}(\bar{p})=$ $(1-\theta) c \bar{p}\left(\frac{1-\widetilde{p}}{\widetilde{p}}\right)$ attracts all types $p>\bar{p}$ and yields a profit. Hence, we must show that $\int_{\bar{p}}^{1}(1-\theta) c \bar{p}\left(\frac{1-\tilde{p}}{\widetilde{p}}\right) d F(p)-\int_{\tilde{p}}^{1}\left(c_{A}+\frac{c p}{\widetilde{p}}\right)<0$ for all $\tilde{p}$ and $\bar{p}$. 
Let $\bar{p}$ maximize this expression conditional on $\widetilde{p}$ and rewrite the expression as

$$
\int_{\bar{p}}^{\widetilde{p}}(1-\theta) c \bar{p}\left(\frac{1-\widetilde{p}}{\widetilde{p}}\right) d F(p)-\int_{\widetilde{p}}^{1}\left\{c_{A}+c\left[\frac{p-(1-\theta) \bar{p}(1-\widetilde{p})}{\widetilde{p}}\right]\right\} d F(p)<0
$$

Because $\frac{c}{d} \leq \bar{p} \leq \widetilde{p}<1$, we have $\int_{\bar{p}}^{\widetilde{p}}(1-\theta) c \bar{p}\left(\frac{1-\tilde{p}}{\widetilde{p}}\right) d F(p) \leq \int_{\frac{c}{d}}^{\widetilde{p}}(1-\theta) c p\left(\frac{1-\tilde{p}}{\tilde{p}}\right) d F(p)$ for any $\bar{p}$ and $\tilde{p}$. Thus, the first term in (18) is smaller than the first term in (17). It remains to show that $\int_{\widetilde{p}}^{1}\left\{c_{A}+c\left[\frac{p-(1-\theta) \bar{p}(1-\tilde{p})}{\widetilde{p}}\right]\right\} d F(p) \geq \int_{\widetilde{p}}^{1}\left\{c_{A}+c[\theta+(1-\theta) p]\right\} d F(p)$. This holds as long as $\frac{p-(1-\theta) \bar{p}(1-\widetilde{p})}{\widetilde{p}} \geq(1-\theta) p+\theta$ for all $p>\widetilde{p} \geq \bar{p}$. This inequality is equivalent to $p>(1-\theta)\left[\hat{p}^{*} p+(1-\widetilde{p}) \bar{p}\right]+\theta \widetilde{p}$. The RHS is a convex combination of points strictly lower than $p$, so this inequality always hold. Hence, for any $\widetilde{p}$, the LHS of (18) is lower than the LHS of (17). Thus, whenever $p^{*}=1$ in the problem with symmetric information, there is no profitable deviation from $p^{*}=1$ and a pooling equilibrium exists.

\section{Proof of Proposition 6}

Proof. Denote by $p_{M, S I}^{*}$ the optimal litigation threshold in Proposition 4 and let $p_{M, A I}^{*}$ the optimal threshold in Theorem $1 .^{25}$ Denote by $\bar{p}$ the solution to $\bar{p}=\frac{1-F(\bar{p})}{f(\bar{p})}$. Note that $p_{M, S I}^{*} \in\left[\frac{c}{d}, 1\right]$ and under Assumption $1 \bar{p} \leq p_{M, A I}^{*}$. Thus, whenever $p_{M, S I}^{*} \leq \bar{p}$ we have $p_{M, S I}^{*} \leq p_{M, A I}^{*}$. Consider first the case $p_{M, S I}^{*} \geq \bar{p}$. Then, $p_{M, S I}^{*} \in \arg \max _{\hat{p}^{*} \in\left[\frac{c}{d}, \infty\right]} \Psi_{S I}\left(\hat{p}^{*}\right)=$ $\arg \max _{\hat{p}^{*} \in[\bar{p}, \infty]} \Psi_{S I}\left(\hat{p}^{*}\right)$. It is easy to see that the objective function in Theorem 1 can be written as $\Psi_{A I}\left(\hat{p}^{*}\right)=\Psi_{S I}\left(\hat{p}^{*}\right)-\Delta\left(\hat{p}^{*}\right)$, where

$$
\Delta\left(\hat{p}^{*}\right)=\frac{(1-\theta) c}{\hat{p}^{*}} \int_{c / d}^{\bar{p}} p f(p) d p+\frac{(1-\theta) c}{\hat{p}^{*}} \int_{\bar{p}}^{\hat{p}^{*}}(1-F(p)) d p+\frac{c}{\hat{p}^{*}} \int_{\hat{p}^{*}}^{1}(1-F(p)) d p .
$$

Consider the problem $p^{*}(\beta)=\arg \max _{\hat{p}^{*} \in[\bar{p}, \infty]} \Psi_{S I}\left(\hat{p}^{*}\right)-\beta \Delta\left(\hat{p}^{*}\right)$, so $p^{*}(0)=p_{M, S I}^{*}$ and $p^{*}(1)=$ $p_{M, A I}^{*}$. By Topkis theorem, when $\Delta^{\prime}\left(\hat{p}^{*}\right)<0$ for all $\hat{p}^{*}$ we have $p^{*}(0) \leq p^{*}(1)$. Note that

$$
\Delta\left(\hat{p}^{*}\right)=\frac{(1-\theta) c}{\hat{p}^{*}}\left[\int_{c / d}^{\bar{p}} p f(p) d p+\int_{\bar{p}}^{1}(1-F(p))\right]+\theta \frac{c}{\hat{p}^{*}} \int_{\hat{p}^{*}}^{1}(1-F(p)) d p .
$$

\footnotetext{
${ }^{25}$ For simplicity, we can assume that the solution of each of these problems is unique. If not, our conclusion holds under the notion of strong set order.
} 
Let $A$ be the term in the bracket, which is independent of $\hat{p}^{*}$. Taking the derivative we get $\Delta^{\prime}\left(\hat{p}^{*}\right)=-\frac{c}{\left(\hat{p}^{*}\right)^{2}}\left[(1-\theta) A+\theta \int_{\hat{p}^{*}}^{1}(1-F(p)) d p\right]-\theta \frac{c}{\hat{p}^{*}}\left(1-F\left(\hat{p}^{*}\right)\right)<0$. 


\title{
ONLINE APPENDIX
}

\section{Liability Insurance: Equilibrium Contracts under Monopoly and Competition}

\author{
Jorge Lemus, Emil Temnyalov and John L. Turner
}

\section{Table of Contents:}

B.1 Insurance for a Risk-Averse Agent

B.1.1 Mean-Variance Preferences

B.1.2 Complete Information

B.1.3 Symmetric Information

B.1.4 Optimal Menu of Contracts under Adverse Selection - Monopoly

B.1.5 Perfect Competition Under Adverse Selection with Risk Aversion

B.2 Control over Settlement Decision

B.3 Bargaining under Incomplete Information

B.4 Alternative Equilibrium Concepts

B.5 Characterization of the Symmetric Information Contract

Jorge Lemus, University of Illinois Urbana-Champaign. jalemus@illinois.edu. Emil Temnyalov, University Technology Sydney. emil.temnyalov@uts.edu.au. John L. Turner, University of Georgia. jlturner@uga.edu. 


\section{B Online Appendix}

\section{B.1 Insurance for a Risk-Averse Agent}

Risk aversion introduces novel elements that are absent in the baseline case. First, insurance reduces the risk of going to litigation. Second, the agent's wealth may determine the agent's level of risk aversion, which affects the equilibrium transfer under bargaining. In addition, generally there is no separability between the cost of insurance for the agent and the settlement payoff. So even in the absence of wealth effects (e.g., CARA utility), the price of insurance may alter the bargaining core. Third, the settlement fee paid by the agent, as well as the willingness to pay for insurance, do not generally have closed-form solutions. As a result, in general, the main analysis the model under risk aversion is not analytically tractable.

Consider a risk-averse agent with initial wealth $w$ covered by an insurance policy $\alpha=$ $\left(\alpha_{S}, \alpha_{L} \alpha_{D}\right)$, bought at some price $Q$, and with preferences over lotteries represented by an increasing and concave Bernouilli utility function $u(\cdot)$. If the third party and the agent go to litigation, the expected payoff of the agent is

$$
u(C E(p, \alpha, Q)) \equiv p u\left(w-c_{A}+\alpha_{L}-d+\alpha_{D}-Q\right)+(1-p) u\left(w-c_{A}+\alpha_{L}-Q\right),
$$

where $C E(p, \alpha, Q)$ denotes the certainty equivalent of the risky litigation outcome under insurance policy $\alpha$ bought at price $Q$. Under risk neutrality, we showed that an uninformed insurer fully cover litigation costs, i.e., $\alpha_{L}=c_{A}$. However, under risk aversion this is not necessarily true. The reason is that $\alpha_{L}$ increases the payoff in both states of the world, which reduces the value of a larger $\alpha_{D}$ to decrease the variance of the lottery. ${ }^{26}$ Additionally, the certainty equivalent of going to litigation is affected by the price of the insurance and the level of wealth of the agent, whereas in the risk-neutral case the agent's wealth and the price of insurance do not affect the decision to litigate.

Under risk aversion parties are also better off by avoiding litigation: they save on litigation

${ }^{26}$ We have $\frac{\partial^{2}}{\partial \alpha_{L} \partial \alpha_{D}} u(C E(p, \alpha, Q))<0$. Under risk-neutrality this cross-derivative is zero. 
cost, and the agent avoids the risky litigation outcome. This means that risk aversion provides stronger settlement incentives to the parties. A feasible settlement agreement is a transfer $T$ from the agent to the third party such that $0<p d-c \leq T$ and $u(C E(p, \alpha, Q)) \leq$ $u\left(w-Q-\max \left\{T-\alpha_{S}, 0\right\}\right)$ or, equivalently,

$$
T_{\min }(p) \equiv p d-c \leq T \leq w-Q-C E(p, \alpha, Q)+\min \left\{\alpha_{S}, T\right\}
$$

If $w-Q-C E(p, \alpha, Q)<0$, the agent will not accept a settlement agreement and parties litigate. If $w-Q-C E(p, \alpha, Q) \geq 0$, the agent accepts a settlement agreement as long as $T \leq T_{\max }(p, \alpha, Q) \equiv w-Q-C E(p, \alpha, Q)+\alpha_{S}$. Without insurance parties always settle because $u(C E(p, 0,0)) \leq u(w-T)$ for any transfer $T \geq p d-c .^{27}$

When $w-Q-C E(p, \alpha, Q) \geq 0$ and $T_{\min }(p) \leq T_{\max }(p, \alpha, Q)$ the agent and the third party settle. In this case, the settlement fee is given by the solution to the maximization of the Nash-product. As in the case of risk neutrality, efficiency of Nash Bargaining implies that settlement transfer must be larger than $\alpha_{S}$, so we can write the problem as:

$$
\begin{aligned}
& T^{\alpha}(p, Q) \in \underset{T}{\arg \max }\left(u\left(w-Q-T+\alpha_{S}\right)-u(C E(p, \alpha, Q))\right)^{\theta}(T-(p d-c))^{1-\theta} \\
& \text { subject to } \max \left\{\alpha_{S}, p d-c\right\} \leq T \leq w-Q-C E(p, \alpha, Q)+\alpha_{S} .
\end{aligned}
$$

Under risk neutrality, we showed that any contract such that $T_{\min }(p)=T_{\max }(p, \alpha, Q)$ is optimal, and under imperfect information the insurer sets $\alpha_{L}=c_{A}$ and $\alpha_{S}=0$. Under risk aversion, however, this result may no longer hold given the non-linearity of $Q+C E(p, \alpha, Q)$, as a function of $Q$ (in the risk neutral case, $Q+C E(p, \alpha, Q)$ is independent of $Q$ ). The price of insurance could increase by reducing the litigation cost coverage $\left(\alpha_{L}<c_{A}\right)$, reducing the payment of the litigation lottery in each state of nature, which increases the value of damage coverage $\left(\alpha_{D}\right)$, which decreases risk. In addition, to increase incentives to settle, the insurer may set a positive $\alpha_{S}$. Covering settlements has two effects. First, increasing $\alpha_{S}$ increases the settlement transfer, which lowers the agent's willingness to pay. Second, increasing $\alpha_{S}$ may provide incentives to settle.

\footnotetext{
${ }^{27}$ To see this, note that $u(C E(p, 0,0)) \leq u(w-\tau)$, where $\tau=p d+c_{A}>p d-c$.
} 
For the general class of risk-averse preferences, the outcome of bargaining may depend upon wealth $w$ and the price of insurance $Q$. Our model is then not analytically tractable for analysis beyond the complete information case. We can simulate outcomes for certain classes of utility functions, but this introduces a taxonomy of possible cases to consider (e.g., increasing risk aversion, decreasing risk aversion, etc.). Analyzing all these cases for a class of distributions of types is beyond the scope of this paper. For this reason, in the next section we focus on one class of risk-preferences that allow us to gain some analytical tractability.

\section{B.1.1 Mean-Variance Preferences}

For mean-variance preferences we can obtain some analytic results. An agent with these preferences evaluates lottery $X$ according to

$$
U(X)=E(X)-\frac{\sigma \operatorname{Var}(X)}{2}
$$

Under insurance policy $\alpha=\left(\alpha_{S}, \alpha_{L}, \alpha_{D}\right)$, the certainty equivalent under litigation is ${ }^{28}$

$$
C E(p, \alpha)=w-\left(c_{A}-\alpha_{L}\right)-p\left(d-\alpha_{D}\right)-\frac{\sigma p(1-p)\left(d-\alpha_{D}\right)^{2}}{2}
$$

The only difference with the risk neutral case is the last term $R P\left(p, \alpha_{D}\right) \equiv \frac{\sigma p(1-p)\left(d-\alpha_{D}\right)^{2}}{2}$, which corresponds to the agent's risk-premium. The bargaining surplus is

$$
S_{B}=\min \left\{T, \alpha_{S}\right\}+c+c_{A}-\alpha_{L}-p \alpha_{D}+R P\left(p, \alpha_{D}\right)
$$

Mirroring the proof of the risk neutral case, it can be shown that $T \geq \alpha_{S}$. Then, the bargaining surplus for type $p$ is simply

$$
S_{B}(p)=\alpha_{S}+c+c_{A}-\alpha_{L}-p \alpha_{D}+R P\left(p, \alpha_{D}\right)
$$

When $S_{B}(p) \geq 0$ the agent of type $p$ and the third party settle, otherwise they litigate. In the risk-neutral case, the bargaining surplus $S_{R N}(p)=\alpha_{S}+c+c_{A}-\alpha_{L}-p \alpha_{D}$ is linear and strictly decreasing in $p$. Under risk aversion, the bargaining surplus is concave and may be

\footnotetext{
${ }^{28}$ The price $Q$ is paid up-front, so under these preferences the term $Q+C E(p, \alpha, Q)$ is independent of $Q$.
} 
non-monotone in $p$, so in principle it is not clear that we can define a threshold type $p^{*} .{ }^{29}$ Figure 10 illustrates the bargaining surplus as a function of the agent's type for the cases of risk neutrality and risk aversion.

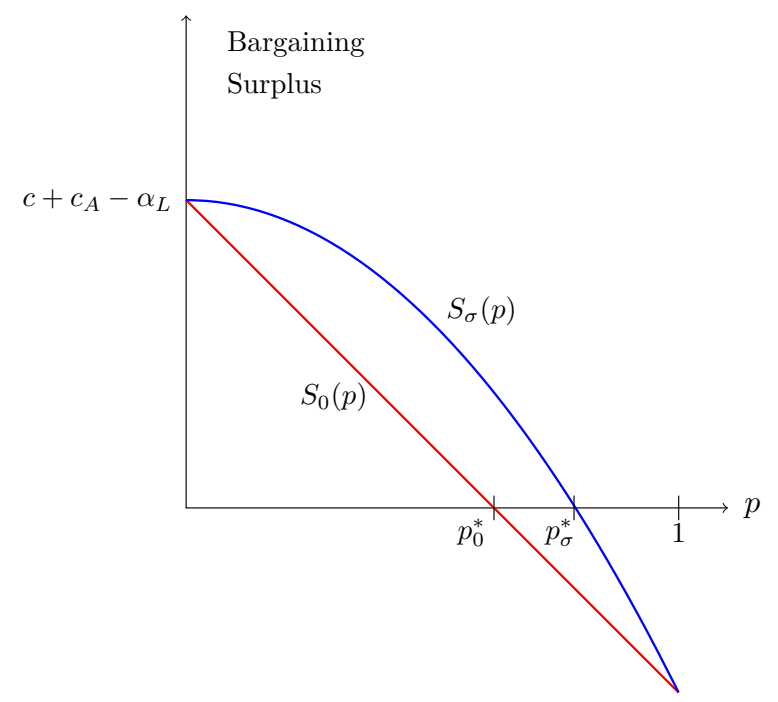

Figure 10: Bargaining surplus as a function of the agent's type for the case of risk neutrality $(\sigma=0)$ and risk aversion $(\sigma>0)$. The thresholds $p_{0}^{*}$ (and $\left.p_{\sigma}^{*}\right)$ correspond to the type that is indifferent between settlement and litigation under risk neutrality (and under risk aversion).

The next lemma guarantees the existence of a unique threshold type $p^{*}$ such that $S_{B}\left(p^{*}\right)=0$.

Lemma 6. There is a unique positive value that solves the equation $S_{B}(p)=0$.

Proof. $S_{B}(0)=\alpha_{S}+c+c_{A}-\alpha_{L}>0$. By concavity, $S_{B}(p)$ has a unique positive root.

Lemma 6 allows us to define a litigation-threshold type $p^{*}$ such that types $p \leq p^{*}$ settle and types $p>p^{*}$ litigate, where $p^{*}$ is the unique positive solution to the equation

$$
p^{*}=\frac{c+c_{A}-\alpha_{L}+\alpha_{S}}{\alpha_{D}}+\frac{R P\left(p^{*}, \alpha_{D}\right)}{\alpha_{D}} .
$$

When $R P\left(p^{*}, \alpha_{D}\right)>0$, the threshold $p^{*}$ is larger than this threshold for a risk neutral agent. This is shown in Figure 10 (for the case $\alpha_{S}=0$, where the threshold for a risk-averse agent $p_{\sigma}^{*}$ is larger than this threshold for a risk neutral agent $p_{0}^{*}$. This is, a risk-averse agent covered by contract $\alpha$ has weakly larger incentives to settle than a risk-neutral agent covered by the

\footnotetext{
${ }^{29}$ By concavity, the bargaining surplus is strictly decreasing for $\sigma<\frac{2 \alpha_{D}}{\left(d-\alpha_{D}\right)^{2}}$.
} 
same contract. Intuitively, risk aversion pushes the agent to settle to avoid the risky litigation outcome. Only under full insurance $\left(\alpha_{D}=d\right)$ this force disappears, i.e., $R P\left(p^{*}, \alpha_{D}\right)=0$. The next result study how a contract $\alpha$ affects the bargaining surplus.

Lemma 7. $S_{B}(\cdot)$ is strictly decreasing in $\alpha_{D}$ and $\alpha_{L}$, and strictly increasing in $\alpha_{S}$. Also, $\frac{\partial S_{B}\left(p^{*}\right)}{\partial p}<0$, and sign $\frac{\partial p^{*}}{\partial \alpha_{j}}=\operatorname{sign} \frac{\partial S_{B}}{\partial \alpha_{j}}$ for $j \in\{S, L, D\}$.

Proof. We have $\frac{\partial S_{B}\left(p^{*}\right)}{\partial p}=\frac{\sigma}{2}\left(d-\alpha_{D}\right)^{2}\left(1-2 p^{*}\right)-\alpha_{D}$. From the definition of $p^{*}$ we have $\frac{\sigma}{2}\left(d-\alpha_{D}\right)^{2}=\frac{p^{*} \alpha_{D}-\left(\alpha_{S}+c+c_{A}-\alpha_{L}\right)}{p^{*}\left(1-p^{*}\right)}$. Replacing we get

$$
\begin{aligned}
\frac{\partial S_{B}\left(p^{*}\right)}{\partial p} & =\left[\frac{p^{*} \alpha_{D}-\left(\alpha_{S}+c+c_{A}-\alpha_{L}\right)}{p^{*}\left(1-p^{*}\right)}\right]\left(1-2 p^{*}\right)-\alpha_{D} \\
& =-\left[\frac{\alpha_{S}+c+c_{A}-\alpha_{L}}{p^{*}}+\frac{R P\left(p^{*}, \alpha_{D}\right)}{1-p^{*}}\right]<0 .
\end{aligned}
$$

To compute the partial derivative with respect to the contract parameters we use that

$$
S_{B}\left(p^{*}\right)=0 \Rightarrow \frac{\partial S_{B}\left(p^{*}\right)}{\partial \alpha_{j}}+\frac{\partial S_{B}\left(p^{*}\right)}{\partial p} \frac{\partial p^{*}}{\partial \alpha_{j}}=0
$$

Given that $\frac{\partial S_{B}\left(p^{*}\right)}{\partial p}<0$, we have sign $\frac{\partial p^{*}}{\partial \alpha_{j}}=\operatorname{sign} \frac{\partial S_{B}}{\partial \alpha_{j}}$ for $j \in\{S, L, D\}$.

Lemma 7 shows that increasing $\alpha_{D}$ or $\alpha_{L}$, or decreasing $\alpha_{S}$ reduces the bargaining surplus. Given that the agent's payment to the third party is proportional to the bargaining surplus, the insurer would like to make the bargaining surplus as small as possible. But this presents a tradeoff for the insurer: decreasing the bargaining will also induce more litigation (by decreasing $p^{*}$ ). The insurer faces this tradeoff when selling insurance agents that are risk neutral or risk averse. The main difference is that damages insurance $\left(\alpha_{D}\right)$ reduces the bargaining surplus and the litigation threshold at a faster rate under risk aversion.

Let $T_{\alpha}(p)=p d-c+(1-\theta) S_{B}(p)$ be the Nash bargaining transfer for contract $\alpha$. An agent of type $p$ pays a settlement transfer equal to $T(p)=\min \left\{T_{\alpha}(p)-\alpha_{S}, 0\right\}$, i.e., the agent is fully covered by the insurer if $T_{\alpha}(p)<\alpha_{S}$. In the risk-neutral case, the Nash bargaining transfer is strictly increasing in $p$, but under risk aversion this transfer may be non-monotone. The reason is that types around $p=1 / 2$ value insurance more than types closer to $p=0$ or 
$p=1$. We need to impose a condition over $\sigma$ to guarantee that the Nash bargaining transfer is increasing in $p$.

Lemma 8. When $\sigma<\frac{2}{d(1-\theta)}$, the Nash bargaining transfer is increasing, and there is a unique threshold type $p^{* *}$ is defined by the condition $p d-c+(1-\theta) S\left(p^{* *}\right)=\alpha_{S}$ such that agents $p \leq p^{* *}$ settle and their settlement offer is fully covered by the insurance.

Proof. We have $T_{\alpha}^{\prime}(p)=d-\alpha_{D}(1-\theta)+(1-\theta) \frac{\sigma\left(d-\alpha_{D}\right)^{2}}{2}(1-2 p)$. It's clear that for any $p$ such that $2 p \leq 1$ we have $T_{\alpha}^{\prime}(p)>0$. Consider then $p$ such that $2 p>1$, so

$$
T_{\alpha}^{\prime}(p)=d-(1-\theta)\left[\alpha_{D}+\frac{\sigma\left(d-\alpha_{D}\right)^{2}}{2}(2 p-1)\right] .
$$

A sufficient condition for this to hold is $d>(1-\theta)\left[\alpha_{D}+\frac{\sigma\left(d-\alpha_{D}\right)^{2}}{2}\right]$. The RHS of this inequality as a function of $\alpha_{D}$ is convex, so the maximum is either $\alpha_{D}=0$ or $\alpha_{D}=d$. When $\alpha_{D}=d$, this holds. When $\alpha_{D}=0$, this holds as long as $\sigma<\frac{2}{d(1-\theta)}$.

For $\sigma$ sufficiently small, $T(p)$ is weakly increasing, which allow us to find a unique solution to the equation $T_{\alpha}(p)=\alpha_{S}$. We will impose this condition for the remainder of the analysis.

Assumption 2. $\sigma<\frac{2}{d(1-\theta)}$.

This expression states that willingness to pay is monotone increasing provided the risk aversion parameter $\sigma$ is less than an amount proportional to the inverse of the damages. Hence, when damages are higher, we need a stronger assumption on the risk parameter $\sigma$.

How do $p^{*}$ and $p^{* *}$ compare? We have that $p^{*}$ solves the equation $S\left(p^{*}\right)=0$ and $p^{* *}$ solves the equation $(1-\theta) S\left(p^{* *}\right)=\alpha_{S}-\left(p^{* *} d-c\right)$ with $\alpha_{S}>p d-c$, so $S\left(p^{* *}\right)=\frac{\alpha_{S}-\left(p^{* *} d-c\right)}{1-\theta}>0$. Given that $S(0)>0$, and $S$ is concave, it is clear that $p^{* *}$ must be smaller than $p^{*}$. Therefore, we can write,

$$
W(p, \alpha)= \begin{cases}T_{0}(p) & \frac{c}{d} \leq p \leq p^{* *} \\ T_{0}(p)+\alpha_{S}-T_{\alpha}(p) & \text { if } p^{* *}<p \leq p^{*} \\ T_{0}(p)-p\left(d-\alpha_{D}\right)-\left(c_{A}-\alpha_{L}\right)-R P\left(p, \alpha_{D}\right) & \text { if } p>p^{*}\end{cases}
$$


Lemma 9. $W(p, \alpha)$ is continuous for any level of risk aversion.

Proof. First, it is easy to verify that $W(p, \alpha)$ is continuous for $p \neq p^{* *}$ or $p \neq p^{*}$. Second, by definition $p^{* *}$ is such that $T_{\alpha}\left(p^{* *}\right)=\alpha_{S}$, so $W(p, \alpha)$ is continuous at $p=p^{* *}$. Third, from the definition of $p^{*}$ we have that $T_{\alpha}\left(p^{*}\right)=p^{*} d-c$ and $p^{*} \alpha_{D}=\alpha_{S}+c+c_{A}-\alpha_{L}+R\left(p^{*}, \alpha_{D}\right)$. Thus, $-p^{*}\left(d-\alpha_{D}\right)-\left(c_{A}-\alpha_{L}\right)-R P\left(p^{*}, \alpha_{D}\right)=\alpha_{S}-T_{\alpha}\left(p^{*}\right)$.

Type $p$ 's willingness to pay for contract $\alpha$ is

$$
W(p, \alpha)= \begin{cases}p d-c+(1-\theta)\left(c+c_{A}+\frac{\sigma d^{2}}{2} p(1-p)\right) & \text { if } \frac{c}{d} \leq p \leq p^{* *}, \\ \theta \alpha_{S}+(1-\theta)\left[\alpha_{L}+p \alpha_{D}+\frac{\sigma \alpha_{D}\left(2 d-\alpha_{D}\right)}{2} p(1-p)\right] & \text { if } p^{* *}<p \leq p^{*}, \\ \alpha_{L}+p \alpha_{D}+\frac{\sigma p(1-p)}{2}\left(\alpha_{D}\left(2 d-\alpha_{D}\right)-\theta d^{2}\right)-\theta\left(c+c_{A}\right) & \text { if } p>p^{*} .\end{cases}
$$

When $\frac{c}{d}<p \leq p^{* *}$ the agent settles and pays nothing - the insurer fully covers the agent's settlement transfer. Thus, the agent's willingness to pay for insurance in this case is $T_{0}(p)$, the amount that an agent without insurance would have paid to TP under a settlement agreement. An agent of type $p^{* *}<p \leq p^{*}$ settles litigation. The willingness to pay of this agent is the sum of the direct lump-sum transfer to cover settlement $\alpha_{S}$ plus the TP's share of the bargaining surplus not captured in the negotiation due to insurance $(1-\theta)\left[\alpha_{L}-\alpha_{S}+p \alpha_{D}+\right.$ $\left.0.5 \sigma \alpha_{D}\left(2 d-\alpha_{D}\right) p(1-p)\right]$. The first component in the term inside the bracket is the value of insurance $\alpha_{L}-\alpha_{S}+p \alpha_{D}$ realized with or without risk aversion. Intuitively, insurance allows the agent to extract additional surplus by improving its own threat point and reducing TP's payoff towards its own threat point. The second component is the risk-premium reduction effect of insurance:

$$
\frac{\sigma p(1-p) \alpha_{D}\left(2 d-\alpha_{D}\right)}{2}=\frac{\sigma p(1-p) d^{2}}{2}-\frac{\sigma p(1-p)\left(d-\alpha_{D}\right)^{2}}{2}
$$

With damages insurance $\alpha_{D}$, the agent faces a loss of just $d-\alpha_{D}$ instead of $d$ when TP wins the case. Because the agent ultimately settles with or without insurance, this gain enters the bargaining surplus directly, and the agent captures share $1-\theta$ of it.

An agent of type $p>p^{*}$ litigates. The term $\alpha_{L}+p \alpha_{D}$ is the direct value of insurance. Because 
the agent litigates, there is no bargaining surplus and it does not share this benefit with the third party.

The second term is the risk-premium reduction:

$$
\frac{p(1-p) \sigma}{2}\left[\left(\alpha_{D}\left(2 d-\alpha_{D}\right)\right)-\theta d^{2}\right]=(1-\theta) \frac{\sigma p(1-p) d^{2}}{2}-\frac{\sigma p(1-p)\left(d-\alpha_{D}\right)^{2}}{2} .
$$

This is lower under litigation than under settlement, by $\theta\left(\frac{\sigma p(1-p)\left(d-\alpha_{D}\right)^{2}}{2}\right)$, because the agent endures the entire variance when it owns insurance (and litigates). When $\theta=0$, the variancereduction effect is the same under settlement and litigation. Intuitively, the variance part of the agent's utility under insurance is the same (for $\theta=0$ ) when the agent settles and when it litigates. As $\theta$ increases, the variance part of the agent's utility under insurance (which enters negatively) stays the same under litigation, but declines under settlement. For sufficiently high $\theta>\frac{d^{2}}{\alpha_{D}\left(2 d-\alpha_{D}\right)}$, the variance-reduction effect is negative.

The third term is the litigation cost effect. This is the part of the agent's payoff under no insurance that accrues from settling and avoiding litigation costs. This is surrendered under litigation.

In contrast to the risk neutral case, $W(p, \alpha)$ may not be increasing in $p$ the region $\left[p^{*}, p^{* *}\right]$. The reason is that agents whose types are around $p=1 / 2$ value insurance more than agents whose types are closer to $p=0$ or $p=1$. Figure 11 shows the shape of the willingness to pay when risk aversion is relatively low (left panel) and high (right panel). Differentiating with respect to $p$, we have

$$
\frac{W(p, \alpha)}{d p}= \begin{cases}d+(1-\theta) \frac{\sigma d^{2}(1-2 p)}{2} & \text { if } \frac{c}{d} \leq p \leq p^{* *} \\ (1-\theta)\left[\alpha_{D}+\frac{\sigma(1-2 p)}{2}\left(\alpha_{D}\left(2 d-\alpha_{D}\right)\right)\right] & \text { if } p^{* *}<p \leq p^{*} \\ \alpha_{D}+\frac{\sigma(1-2 p)}{2}\left(\alpha_{D}\left(2 d-\alpha_{D}\right)-\theta d^{2}\right) & \text { if } p>p^{*}\end{cases}
$$

Lemma 10. $W(p, \alpha)$ is strictly increasing in $p$ for $\sigma \leq \frac{1}{d}$.

Proof. It can be shown that $\left.\frac{W(p, \alpha)}{d p}\right|_{p^{* *}<p \leq p^{*}}>0$ is the hardest condition to satisfy. Using that $\alpha_{D} \leq d$, it is easy to see that this term is positive when $\sigma \leq \frac{1}{d}$. 
Note that Assumption 2 does not guarantee the condition in Lemma 10.
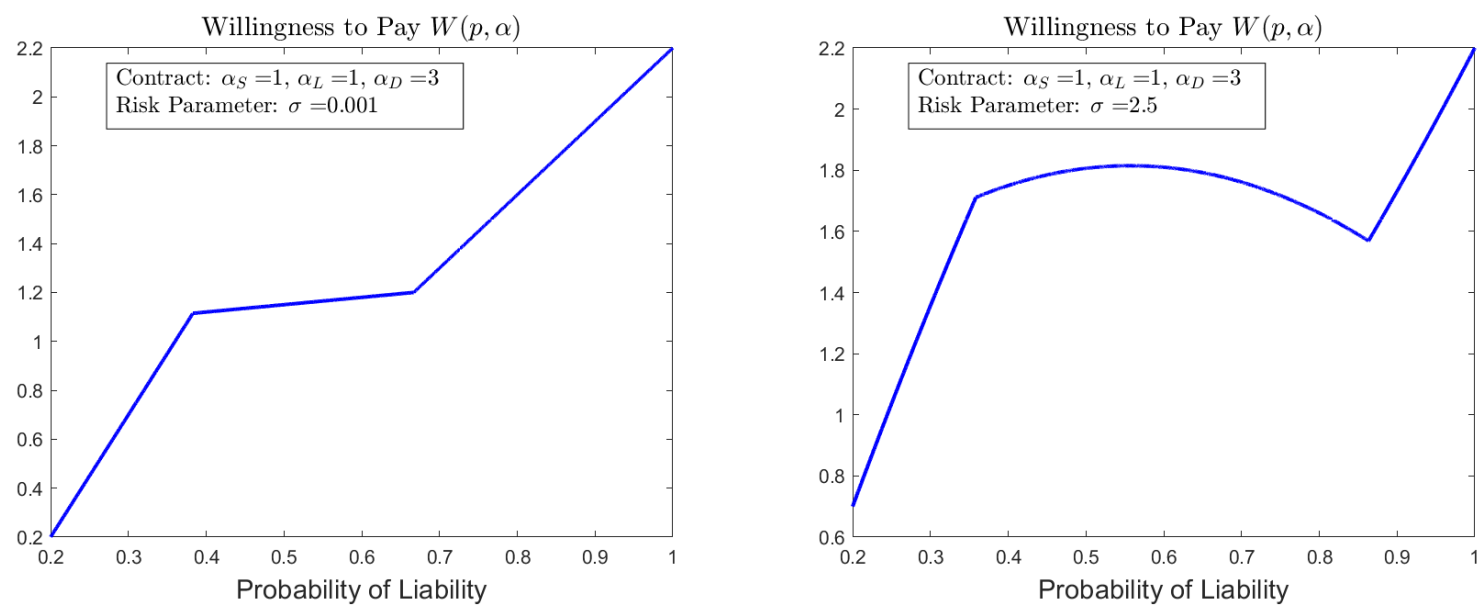

Figure 11: $W(p, \alpha)$ for contract $\alpha_{S}=1, \alpha_{L}=1$, and $\alpha_{D}=3$ and model parameters: $c=c_{A}=1$, $d=5, \theta=0.8$.

The net surplus from serving type $p$ with policy $\alpha$ is

$$
(W-K)(p, \alpha)= \begin{cases}p d-c+(1-\theta)\left(c+c_{A}+\frac{\sigma d^{2}}{2} p(1-p)\right)-\alpha_{S} & \text { if } \frac{c}{d} \leq p \leq p^{* *} \\ (1-\theta)\left[\alpha_{L}-\alpha_{S}+p \alpha_{D}+\frac{\sigma \alpha_{D}\left(2 d-\alpha_{D}\right)}{2} p(1-p)\right] & \text { if } p^{* *}<p \leq p^{*} \\ -\theta\left(c+c_{A}\right)+\frac{\sigma p(1-p)}{2}\left(\alpha_{D}\left(2 d-\alpha_{D}\right)-\theta d^{2}\right) & \text { if } p>p^{*}\end{cases}
$$

In contrast to the risk neutral case, it is not always true that the net surplus is negative for types that litigate $\left(p>p^{*}\right)$; for instance, this term will be positive when $\theta$ is small. Using the definition of $p^{*}$, we can write

$$
(W-K)(p, \alpha)= \begin{cases}p d-c+(1-\theta)\left(c+c_{A}+R P(p, 0)\right)-\alpha_{S} & \text { if } \frac{c}{d} \leq p \leq p^{* *} \\ (1-\theta)\left[c+c_{A}+R P(p, 0)+\left(p-p^{*}\right) \alpha_{D}+R P\left(p^{*}, \alpha_{D}\right)-R P\left(p, \alpha_{D}\right)\right] & \text { if } p^{* *}<p \leq p^{*} \\ -\theta\left(c+c_{A}\right)+(1-\theta) R P(p, 0)-R P\left(p, \alpha_{D}\right) & \text { if } p>p^{*}\end{cases}
$$

Equation 27 describes the willingness to pay as a function of the threshold types $p^{*}$ and $p^{* *}$ generated by contract $\alpha$.

Lemma 11. Consider two contracts: $\alpha=\left(\alpha_{S}, \alpha_{L}, \alpha_{D}\right)$ that generates threshold $p^{*}$ and $p^{* *}$, 
and $\hat{\alpha}=\left(\hat{\alpha}_{S}, \hat{\alpha}_{L}, \alpha_{D}\right)$ that generates threshold $\hat{p}^{*}=p^{*}$ and $\hat{p}^{* *}<p^{* *}$. If $\hat{\alpha}_{S}<\alpha_{S}$, then $(W-K)(p, \alpha)=(W-K)(p, \hat{\alpha})$ for all $p \in\left[p^{* *}, 1\right]$ and $(W-K)(p, \alpha)<(W-K)(p, \hat{\alpha})$ for all $p<p^{* *}$.

Proof. Both contracts generate the same litigation threshold $p^{*}$ and both contracts cover the same amount in damages $\alpha_{D}$. Thus, we have that $(W-K)(p, \alpha)=(W-K)(p, \hat{\alpha})$ for all $p \in\left[p^{* *}, 1\right]$. For any $p \leq \hat{p}^{* *}$ we have that $(W-K)(p, \alpha)<(W-K)(p, \hat{\alpha})$ because $\hat{\alpha}_{S}<\alpha_{S}$. Finally, consider any $p \in\left(\hat{p}^{* *}, p^{* *}\right)$. In this region, $(W-K)(p, \alpha)<(W-K)(p, \hat{\alpha})$ is equivalent to

$$
p d-c-\alpha_{S}<(1-\theta)\left[\left(p-p^{*}\right) \alpha_{D}+R P\left(p^{*}, \alpha_{D}\right)-R P\left(p, \alpha_{D}\right)\right] .
$$

It is easy to see that this inequality corresponds to $T(p)<\alpha_{S}$, which holds for any $p<p^{* *}$.

Lemma 11 implies that given to contracts that induce the same threshold $p^{*}$ and cover the same amount for damages $\alpha_{D}$, the insurer will prefer the contract with the smallest $p^{* *} .30$ Thus, we can show that some contracts are dominated.

Proposition 7. For a given contract $\alpha=\left(\alpha_{S}, \alpha_{L}, \alpha_{D}\right)$ define the contract $\hat{\alpha}=\left(\max \left\{\alpha_{S}-\right.\right.$ $\left.\left.\alpha_{L}, 0\right\}, \max \left\{\alpha_{L}-\alpha_{S}, 0\right\}, \alpha_{D}\right)$. Then, $(W-K)(p, \alpha) \leq(W-K)(p, \hat{\alpha})$ for any $p$.

Proof. First, note that both contracts $\alpha$ and $\hat{\alpha}$ generate the same threshold $p^{*}$ because $\alpha_{S}-\alpha_{L}=\max \left\{\alpha_{S}-\alpha_{L}, 0\right\}-\max \left\{\alpha_{L}-\alpha_{S}, 0\right\}$. Second, note that $\max \left\{\alpha_{S}-\alpha_{L}, 0\right\} \leq \alpha_{S}$. Thus, by Lemma 11 we have $(W-K)(p, \alpha) \leq(W-K)(p, \hat{\alpha})$ for any $p$.

Proposition 7 implies that, if the goal is to maximize $W-K$ pointwise, the insurer will never offer contracts that cover simultaneously settlements and litigation costs, because these contracts are pointwise dominated. Thus, we can focus on contracts of the form $\left(\alpha_{S}, 0, \alpha_{D}\right)$ or $\left(0, \alpha_{L}, \alpha_{D}\right)$.

Under risk neutrality, Proposition 3 (in the main text) shows that we can go one step further: To maximize $(W-K)$ pointwise in $p$ we can restrict to contracts of the form $\alpha=\left(0, c_{A}, c / p^{*}\right)$,

\footnotetext{
${ }^{30}$ Given that $T^{\prime}(p)>0$ we have $\frac{\partial p^{* *}}{\partial \alpha_{S}}=\frac{\theta}{T^{\prime}\left(p^{* *}\right)}>0$, so lowering $\alpha_{S}$ will also lower $p^{* *}$.
} 
for some $p^{*}>c / d$. The reason is that for any fixed $p^{*}$ and any contract that satisfy $p^{*} \alpha_{D}=$ $c+c_{A}-\alpha_{L}+\alpha_{S}$ we have: $\frac{\partial(W-K)}{\partial \alpha_{D}}<0$ for $p \in\left(p^{* *}, p^{*}\right)$ and zero otherwise; $\frac{\partial(W-K)}{\partial \alpha_{S}}<0$ for $p<p^{* *}$ and zero otherwise; and, $\frac{\partial(W-K)}{\partial \alpha_{L}}=0$ for all $p$. Thus, the insurer has a preference for reducing $\alpha_{S}$ and $\alpha_{D}$ as much as possible, as long as the contract parameters satisfy $\alpha_{L}=c_{A}+c-p^{*} \alpha_{D}+\alpha_{S}$ with $\alpha_{L} \leq c_{A}$. Therefore, the insurer sets $\alpha_{S}=0$ and $\alpha_{D}=\frac{c}{p^{*}}$.

Under risk aversion, for any fixed $p^{*}$ and any contract that satisfy $p^{*} \alpha_{D}=c+c_{A}-\alpha_{L}+$ $\alpha_{S}+R P\left(\alpha_{D}, p^{*}\right)$ we still have that: $\frac{\partial(W-K)}{\partial \alpha_{S}}<0$ for $p<p^{* *}$ and zero otherwise; and that $\frac{\partial(W-K)}{\partial \alpha_{L}}=0$ for all $p$. However, the partial derivative with respect to $\alpha_{D}$ is no longer weakly negative:

$\frac{\partial(W-K)}{\partial \alpha_{D}}(p, \alpha)= \begin{cases}0 & \text { if } \frac{c}{d} \leq \frac{c}{d} \leq p \leq p^{* *} \\ (1-\theta)\left[\left(p-p^{*}\right) \alpha_{D}-\sigma\left(d-\alpha_{D}\right)\left(p^{*}\left(1-p^{*}\right)-p(1-p)\right)\right] & \text { if } p^{* *}<p \leq p^{*} \\ \sigma\left(d-\alpha_{D}\right) p(1-p) & \text { if } p>p^{*}\end{cases}$

We have $\frac{\partial(W-K)}{\partial \alpha_{D}}(p, \alpha)<0$ for $p \in\left[p^{*}, p^{* *}\right]$ and, when $\alpha_{D}<d, \frac{\partial(W-K)}{\partial \alpha_{D}}(p, \alpha)>$ 0for $p>p^{*}$. Thus, in contrast to the risk neutral case, it is not true that the insurer wants to reduce $\alpha_{D}$ as much as possible. This implies that even under Assumption 2 there may not exist a contract that maximizes $W-K$ pointwise in $p$.

The insurer's cost of serving type $p$ with policy $\alpha$ depends on the agent's level of risk aversion through the threshold $p^{*}$.

$$
K(p, \alpha)= \begin{cases}\alpha_{S} & \text { if } p \leq p^{*} \\ \alpha_{L}+p \alpha_{D} & \text { if } p>p^{*}\end{cases}
$$

In contrast to the risk neutral case, the contract that maximizes $W-K$ at $p$ for a risk-averse agent, for a fixed litigation threshold $p^{*}$, in some cases depends on $p$; this also explains why under risk aversion it is impossible to find a contract that maximizes $W-K$ pointwise for all $p$. To further hone the contrast between risk neutrality and risk aversion we present an example. In the example, we explain intuitively how to find the contract that maximizes $(W-K)$ at $p$ for a fixed litigation threshold $p^{*}$. 


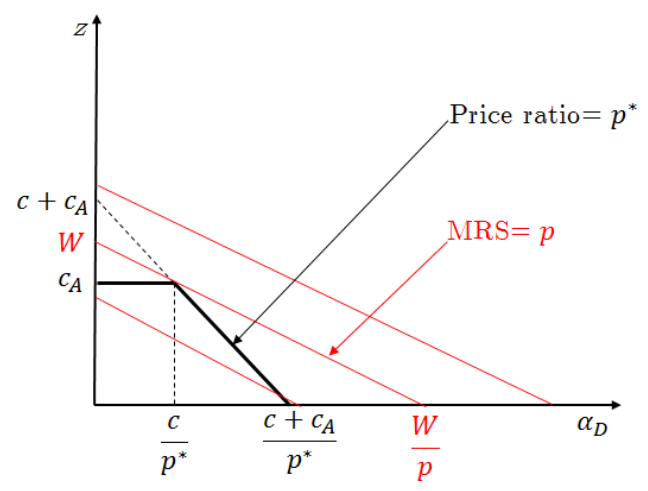

Figure 12: Maximization of $W-K$ for $p<p^{*}$ in the case of risk neutrality.

Example. Consider the set of contracts that generate a fixed litigation threshold $p^{*}$. Within that set of contracts let us find the contract that maximizes $(W-K)$ at some point $p$. Let $z \equiv \alpha_{L}-\alpha_{S} \in\left(\infty, c_{A}\right]$

1. Risk neutrality. This case is easily illustrated using a simple consumer choice framework, where the "consumer" is the agent+insurer $(\mathrm{A}+\mathrm{I})$ and whose utility is $W-K$ and must choose over the "budget set" $z=c_{A}+c-\alpha_{D} p^{*}$ subject to $z \leq c_{A}$. This constraint is represented by the two-piece black line in Figure 12. When $p<p^{*}, \mathrm{~A}+\mathrm{I}$ 's willingness to pay $(W-K)(p)=(1-\theta)\left(z+p \alpha_{D}\right)$ is represented by the red indifference curves, which have slope $p$. As a result, the optimal mix of $z$ and $\alpha_{D}$ is always at the corner where $z=c_{A}$, which implies that $\alpha_{L}=c_{A}$ and $\alpha_{S}=0$. Intuitively, $(\mathrm{A}+\mathrm{I})$ 's marginal rate of substitution $p$ is lower than $p^{*}$. As a result, litigation costs insurance is more valuable given the tradeoff implied by $p^{*}$, and the optimal mix of $z$ and $\alpha_{D}$ is always at the corner where $\alpha_{L}=c_{A}$. Finally, when $p>p^{*}$ (i.e., types that litigate) we have that $(W-K)(p)$ is always negative and is constant, so it is affected by the choice of $p^{*}$ but is otherwise unaffected by the choices of $z$ and $\alpha_{D}$.

2. Risk aversion. Fixing $p^{*}$ yields a non-linear constraint illustrated in Figure 13 . The $z$-axis intercept of the formula for the $S\left(p^{*}\right)=0$ constraint is higher because of the risk premium. The additional constraint $z \leq c_{A}$ still must hold, and it binds at an $\alpha_{D}>\frac{c}{p^{*}}$ because of the risk premium. In addition, the risk premium makes the constraint a convex function of $\alpha_{D}$. The slope of this constraint is $-p^{*}\left[1+\left(1-p^{*}\right) \sigma\left(d-\alpha_{D}\right)\right]$.

Indifference curves for an agent of type $p$-where $p<p^{*}$ - similarly have a convex shape, 


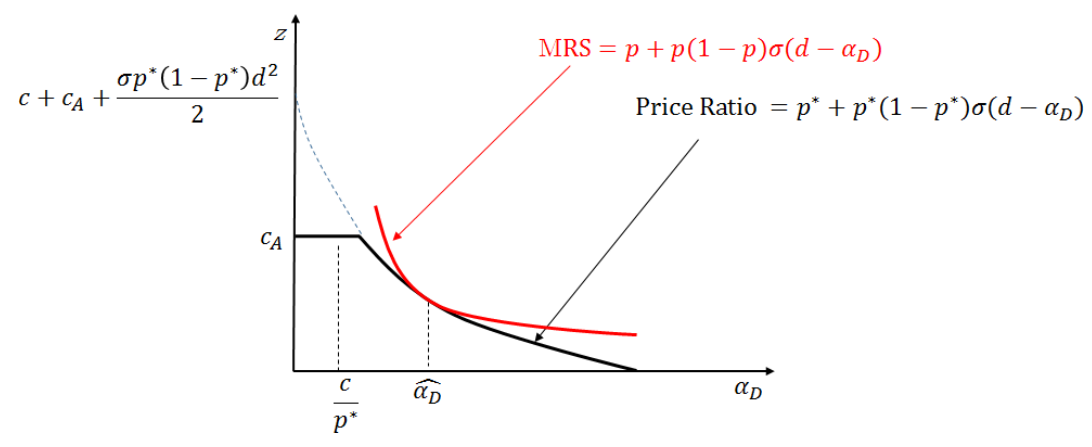

Figure 13: Maximization of $W-K$ for $p<p^{*}$ in the case of risk aversion.

with slope $-p\left[1+(1-p) \sigma\left(d-\alpha_{D}\right)\right]$. The indifference curve is tangent to the constraint at

$$
\hat{\alpha}_{D}=d-\left[\frac{p-p^{*}}{\sigma\left(p^{*}\left(1-p^{*}\right)-p(1-p)\right)}\right] .
$$

If this tangency occurs for $z<c_{A}$, as in Figure 13, then the $\hat{\alpha}_{D}$ given in (29) optimizes $W$ conditional on $p^{*}$. Otherwise the optimal coverage obtains at the upper boundary. Now, it is impossible for $\hat{\alpha}_{D}$ to be negative. When this condition implies a negative $\hat{\alpha}_{D}$, then we know that the $\alpha_{L}=c_{A}$ kink binds. We can use this condition to determine a restriction on $\sigma$ that guarantees that this kink binds:

$$
\sigma<\frac{1}{d}\left(\frac{1}{p^{*}+p-1}\right)
$$

Because we restrict attention to cases where the second fraction yields a positive number $(p$ closer to .5 than $p^{*}$ ), and to cases where $p \leq p^{*}$, the lowest that the second fraction can be is 1 . Hence, we need only restrict attention to $\sigma<\frac{1}{d}$ to guarantee that $z=\alpha_{C}=c_{A}$ is pointwise optimal for types that settle.

For higher $\sigma$, of course, this is not the case. To see the intuition for that case most clearly, let $p^{*}>.5$. First, note that the difference in the slopes of the curves in Figure 13 depend both on $p$ and $p(1-p)$. If $p<1-p^{*}$, then $p$ is so low that it is farther from $p=.5$ than $p^{*}$. Then both terms in the slope are of lower magnitude for the indifference curves than for the constraint. The indifference curves are then shallower than the constraint everywhere, so the optimal insurance occurs at the $\alpha_{L}=c_{A}$ kink. As $p$ increases, it eventually reaches a point where it is closer to .5 than $p^{*}$. Then, the second term in the slope is of greater magnitude 
for the indifference curve than for the constraint. It is then possible for the red indifference curve to be steeper than the constraint at low $\alpha_{D}$ and shallower at high $\alpha_{D}$.

For types that litigate, a lower $\alpha_{D}$ exposes the agent to more risk when it litigates, which reduces $W-K$ for $p>p^{*}$. Recall total $\mathrm{A}+\mathrm{I}$ profit for types that litigate is

$$
W-K=-\theta\left(c+c_{A}\right)+\frac{\sigma p(1-p)}{2}\left(\alpha_{D}\left(2 d-\alpha_{D}\right)-\theta d^{2}\right)
$$

Obviously, this is maximized for the highest level of damages, $\alpha_{D}=d$, and this is independent of $p^{*}$ because $\alpha_{S}$ is not bounded above. The particular value of $p^{*}$ does matter for $z$ through the $S\left(p^{*}\right)=0$ constraint, which implies $z=c+c_{A}-p^{*} d$.

Table 2 shows the optimal policy for type $p$ within the set of contracts that generate a litigation threshold $p^{*}$ (in the table, $p^{*}=0.8$ ). Under risk neutrality $(\sigma=0)$, each type $p$ optimally is allocated the same insurance, $\alpha_{L}=c_{A}$ and $\alpha_{D}=\frac{c}{p^{*}}$. This is strictly optimal for types that settle, and weakly optimal for types that litigate.

For low $\sigma=0.1$, each type that settles optimally receives the same level of litigation costs insurance as under risk neutrality. Because of the risk premium, the implied level of damages insurance is higher. And, more importantly, each type that litigates now optimally receives maximum damages coverage $\alpha_{D}=5$, and the implied $z=-2$ for all $p>$.8. Hence, even for the low- $\sigma$ case, there is no contract that maximizes $W-K$ pointwise in $p$.

For high $\sigma=1.25$, the lack of pointwise maximization is even more pronounced. There are essentially three different regions of $p$. For low $p \leq .4875$, the $\alpha_{L}=c_{A}$ kink binds. For $p \in\left(.4875, p^{*}\right]$, the optimal level of $z$ is below $c_{A}$. It is positive for $p \in(.4875, .6]$, and negative for $p \in\left(.6, p^{*}\right]$. For $p>p^{*}$, types litigate. Essentially, the ability to sell coverage for settlements slacks the constraint on how big $\alpha_{D}$ can be, which is valuable when $\sigma$ and $p$ are high. As in the $\sigma=0.1$ case, each type that litigates now receives maximum damages coverage $\alpha_{D}=5$, and the implied $z=-2$ for all $p>$.8. Note that damages coverage strictly increases for $p \in\left[.4875, p^{*}\right]$.

It turns out that the function in (29) is generally monotone increasing in $p$ for $p \in\left[1-p^{*}, p^{*}\right]$. 
After some algebra, we find

$$
\frac{d \hat{\alpha}_{D}}{d p}=\frac{1}{\sigma\left(p^{*}+p-1\right)^{2}}
$$

The implication is that there is no peak in $\hat{\alpha}_{D}$ at $p=.5$. Intuitively, increases in $p$ above .5 increase the direct value of insurance and decrease the risk-premium reduction. But the former effect dominates.

\begin{tabular}{|rrrrrrr|}
\multicolumn{4}{c}{$\sigma=0$} & \multicolumn{2}{c}{$\sigma=0.1$} & \multicolumn{2}{c|}{$\sigma=1.25$} \\
\hline$p$ & $z$ & $\alpha_{D}$ & $z$ & $\alpha_{D}$ & $z$ & $\alpha_{D}$ \\
0.3 & 1.00 & 1.25 & 1.00 & 1.38 & 1.00 & 2.22 \\
0.4 & 1.00 & 1.25 & 1.00 & 1.38 & 1.00 & 2.22 \\
0.5 & 1.00 & 1.25 & 1.00 & 1.38 & 0.85 & 2.33 \\
0.6 & 1.00 & 1.25 & 1.00 & 1.38 & 0.00 & 3.00 \\
0.7 & 1.00 & 1.25 & 1.00 & 1.38 & -0.46 & 3.40 \\
0.8 & 1.00 & 1.25 & 1.00 & 1.38 & -0.75 & 3.67 \\
0.9 & 1.00 & 1.25 & -2.00 & 5.00 & -2.00 & 5.00 \\
1.0 & 1.00 & 1.25 & -2.00 & 5.00 & -2.00 & 5.00 \\
\hline
\end{tabular}

Table 1: Contract that maximizes $W-K$ for type $p$, conditional on $p^{*}=.8, \sigma=1.25, c=c_{A}=1$, $\theta=0.8, d=5$.

To summarize, pointwise maximization obtains under risk neutrality but not under risk aversion. With low levels of $\sigma$, litigation costs coverage remains more valuable than damages insurance for types that settle. But not for types that litigate. With higher levels of $\sigma$, litigation costs coverage is not necessarily more valuable than damages insurance for types that settle.

\section{B.1.2 Complete Information}

If the insurer is informed about the agent's type, then it will offer a contract that targets that type. This is the same result under risk neutrality.

Proposition 8. The optimal contract for type $p$ under complete information is any contract such that $p^{*}(\alpha)=p$.

Proof. We say that contract $\alpha$ targets $p$ if $S(p)=0$, i.e., the litigation threshold implied by this contract is $p^{*}=p$. 
First, by Lemma 11 we can restrict to contracts such that $p>p^{* *}$. If not, we can always reduce $\alpha_{S}$ to achieve this condition. Second, the value of $(W-K)(p, \alpha)$ for a contract $\alpha$ that targets $p$ is independent of the contract parameters and equal to

$$
\pi_{T} \equiv(1-\theta)\left[c+c_{A}+\frac{\sigma d^{2}}{2} p(1-p)\right]
$$

Third, under complete information, the optimal contract for type $p$ does not induce type $p$ to litigate. If type $p$ litigates, it means the insurer offers some contract targets $p^{*}$ with $p^{*}<p$. The value of $W-K$ for this contract at type $p$ is then

$$
\pi_{L}=-\theta\left(c+c_{A}\right)+(1-\theta) R P(p, 0)-R P\left(p, \alpha_{D}\right)
$$

while Equation 33 is the value of $W-K$ at $p$ for a contract that targets type $p$. Note that

$$
\begin{aligned}
\pi_{T}-\pi_{L} & =(1-\theta)\left(c+c_{A}\right)+(1-\theta) R P(p, 0)+\theta\left(c+c_{A}\right)-(1-\theta) R P(p, 0)+R P\left(p, \alpha_{D}\right) \\
& =\left(c+c_{A}\right)+R P\left(p, \alpha_{D}\right)>0 .
\end{aligned}
$$

Therefore, it is never optimal to induce type $p$ to litigate under complete information, so the optimal contract for type $p$ should be such that $p^{* *}<p \leq p^{*}$.

Fourth, $(W-K)(p, \hat{\alpha})$ is higher for a contract $\hat{\alpha}$ that targets type $\hat{p}>p$, than for a contract $\alpha$ that targets $p$ iff

$$
(p-\hat{p}) \widehat{\alpha}_{D}+R P\left(\hat{p}, \widehat{\alpha}_{D}\right)-R P\left(p, \widehat{\alpha}_{D}\right)>0
$$

where $\widehat{\alpha}_{D}$ is the contract parameter associated to contract $\hat{p}$. This can be written as

$$
(\hat{p}-p) \widehat{\alpha}_{D}<\frac{\sigma}{2}\left(d-\widehat{\alpha}_{D}\right)^{2}[\hat{p}(1-\hat{p})-p(1-p)]
$$

Note that $\hat{p}(1-\hat{p})-p(1-p)=(\hat{p}-p)(1-(\hat{p}+p))$, and given that $p<\hat{p}$ we have

$$
\widehat{\alpha}_{D}<\frac{\sigma}{2}\left(d-\widehat{\alpha}_{D}\right)^{2}(1-(\hat{p}+p))
$$

Thus, for any $p>0.5$, this condition never holds because the RHS is negative. Consider 
$p \leq 0.5$. The RHS of the inequality is

$$
\frac{\sigma}{2}\left(d-\widehat{\alpha}_{D}\right)^{2}\left(1-\left(\hat{p}+p^{*}\right)\right) \leq \frac{\sigma}{2}\left(d-\widehat{\alpha}_{D}\right)^{2}(1-\hat{p})
$$

By the definition of $\hat{p}$ we have:

$$
\frac{\sigma}{2}\left(d-\widehat{\alpha}_{D}\right)^{2} \hat{p}(1-\hat{p})=\hat{p} \hat{\alpha}_{D}-\left(\hat{\alpha}_{S}+c+c_{A}-\hat{\alpha}_{L}\right)<\hat{p} \hat{\alpha}_{D}
$$

This implies that

$$
\frac{\sigma}{2}\left(d-\widehat{\alpha}_{D}\right)^{2}(1-\hat{p})<\hat{\alpha}_{D}
$$

Therefore, there is no contract $\hat{\alpha}$ that generates a litigation threshold $\hat{p}$, with $\hat{p}>p$, such that $(W-K)(p, \hat{\alpha})>W-K(p, \alpha)$, where $\alpha$ is any contract that targets type $p$.

Just like in the case of risk neutrality, Proposition 8 shows that the optimal contract under complete information for type $p$ is any the contracts that targets this type. We have multiple contracts that are optimal, in fact, any contract such that $S(p)=0$.

\section{B.1.3 Symmetric Information}

Proposition 7 implies that we need to search for contracts where either $\alpha_{S}=0$ or $\alpha_{D}=0$. The problem solved be the insurer(s) is to find a contract $\alpha$ solution to

$$
\max _{\left\{\alpha=\left(\alpha_{S}, \alpha_{L}, \alpha_{D}\right)\right\}} \int_{c / d}^{1}[W(p, \alpha)-K(p, \alpha)] d F(p)
$$

subject to $\alpha_{L} \cdot \alpha_{S}=0$. Table 2 shows a numerical simulation for the optimal contract under symmetric information. The table shows that for low values of risk aversion the optimal contract shares similar characteristics with the optimal contract under risk neutrality: $\alpha_{S}=0, \alpha_{L}=c_{A}$ and $\alpha_{D} \geq c / p^{*}$. Note, however, that in general $\alpha_{D}>c / p^{*}$. The reason for the insurer to increase $\alpha_{D}$ when the agent is risk averse - relative to the case where the agent is risk neutral - is to protect the agent against the uncertain litigation outcome, which makes the agent tougher in the negotiation with the third party. However, increasing $\alpha_{D}$ too much also induces more litigation. To counteract the increase in litigation, the insurer 
does not cover all the litigation costs; and when not covering litigation costs is not enough to deter the agent from going to litigation, the insurer begins to cover settlements $\left(\alpha_{S}>0\right)$.

\begin{tabular}{|rrrrr|}
\hline \multicolumn{5}{c}{$F(p)=1-(1-p)^{2}$} \\
\hline $10^{-8}$ & $\alpha_{S}$ & $\alpha_{L}$ & $\alpha_{D}$ & $p^{*}$ \\
0.1 & 0 & 1 & 1.01 & 0.995 \\
0.5 & 0 & 1 & 1.03 & 0.983 \\
0.6 & 0 & 1 & 1.245 & 0.947 \\
0.7 & 0 & 1 & 1.385 & 0.939 \\
0.8 & 0 & 1 & 1.460 & 0.925 \\
0.9 & 0 & 0.97 & 1.550 & 0.920 \\
1 & 0 & 0.63 & 1.880 & 0.921 \\
1.5 & 0.23 & 0 & 2.750 & 0.917 \\
2 & 0.26 & 0 & 2.910 & 0.905 \\
\hline
\end{tabular}

\begin{tabular}{|rrrrr|}
\hline \multicolumn{6}{c}{$F(p)=p^{0.5}$} \\
\hline $10^{-8}$ & $\alpha_{S}$ & $\alpha_{L}$ & $\alpha_{D}$ & $p^{*}$ \\
0.1 & 0 & 1 & 1 & 1 \\
0.5 & 0 & 1 & 1 & 1 \\
0.6 & 0 & 0.88 & 1.12 & 1 \\
0.7 & 0 & 0.33 & 1.68 & 1 \\
0.8 & 0.09 & 0 & 2.09 & 1 \\
0.9 & 0.24 & 0 & 2.24 & 1 \\
1 & 0.26 & 0 & 2.26 & 1 \\
1.5 & 0.36 & 0 & 2.36 & 1 \\
2 & 0.44 & 0 & 2.44 & 1 \\
\hline
\end{tabular}

Table 2: Optimal contract under symmetric information for different values of $\sigma$ and different distribution of types. Parameters $c=c_{A}=1, \theta=0.8, d=5$.

\section{B.1.4 Optimal menu of contracts under adverse selection - Monopoly}

We restrict our attention to direct revelation mechanisms: the insurer allocates contract $\alpha(\hat{p})=\left(\alpha_{S}(\hat{p}), \alpha_{L}(\hat{p}), \alpha_{D}(\hat{p})\right)$ at cost $T(\hat{p})$ to an agent reveals a type $\hat{p}$. The insurer chooses functions $\alpha:[c / d, 1] \rightarrow[0, \infty) \times\left[0, c_{A}\right] \times[0, d]$ and $T:[c / d, 1] \rightarrow[0, \infty)$ to solve

$$
\max _{\alpha(\cdot), T(\cdot)} \int_{c / d}^{1}[T(p)-K(p, \alpha(p))] f(p) d p
$$

subject to truthful revelation (incentive compatibility)

$$
U(p)=\max _{\hat{p} \in[c / d, 1]} W(p, \alpha(\hat{p}))-T(\hat{p})
$$

and participation $U(p) \geq 0$. By the envelop theorem we have $U(p)-U(c / p)=\int_{c / d}^{p} \frac{\partial W(s, \alpha(s))}{\partial p} d s$, which is equivalent to

$$
T(p)=W(p, \alpha(p))-\int_{c / d}^{p} \frac{\partial W(s, \alpha(s))}{\partial p} d s+U(c / p)
$$


Replacing in the objective function we obtain

$$
\max _{\alpha(\cdot)} \int_{c / d}^{1}\left[W(p, \alpha(p))-K(p, \alpha(p))-\int_{c / d}^{p} \frac{\partial W(s, \alpha(s))}{\partial p} d s-U(c / d)\right] f(p) d p
$$

It is clear that the monopolist sets $U(c / d)=0$. Using the standard change of variables we get the following problem:

$$
\max _{\alpha(\cdot)} \int_{c / d}^{1}\left[W(p, \alpha(p))-K(p, \alpha(p))-\frac{\partial W(p, \alpha(p))}{\partial p}\left(\frac{1-F(p)}{f(p)}\right)\right] f(p) d p .
$$

In a "standard problem" of mechanism design, incentive compatibility requires an increasing allocation. Our problem differs from the standard case because the allocation is multidimensional, although the private information is single-dimensional.

Lemma 12. Incentive compatibility requires

$$
\sum_{i=1}^{N} \frac{\partial W(s, \alpha(t))}{\partial p \partial \alpha_{i}} \alpha_{i}^{\prime}(t)=\frac{\partial W(s, \alpha(t))}{\partial p \partial \alpha_{S}} \alpha_{S}^{\prime}(t)+\frac{\partial W(s, \alpha(t))}{\partial p \partial \alpha_{L}} \alpha_{L}^{\prime}(t)+\frac{\partial W(s, \alpha(t))}{\partial p \partial \alpha_{D}} \alpha_{D}^{\prime}(t) \geq 0, \quad \forall s, t .
$$

Proof. For any $p, \hat{p}$ for incentive compatibility requires:

$$
W(p, \alpha(p))-T(p) \geq W(p, \alpha(\hat{p}))-T(\hat{p}) .
$$

Let $\Delta(p, \hat{p})=W(p, \alpha(p))-T(p)-[W(p, \alpha(\hat{p}))-T(\hat{p})]$. We have

$$
\begin{aligned}
\Delta(p, \hat{p}) & =W(p, \alpha(p))-T(p)-[W(\hat{p}, \alpha(\hat{p}))-T(\hat{p})+W(p, \alpha(\hat{p}))-W(\hat{p}, \alpha(\hat{p}))] . \\
& =W(p, \alpha(p))-T(p)-[W(\hat{p}, \alpha(\hat{p}))-T(\hat{p})]-[W(p, \alpha(\hat{p}))-W(\hat{p}, \alpha(\hat{p}))] \\
& =\int_{c / d}^{p} \frac{\partial W(s, \alpha(s))}{\partial p} d s-\int_{c / d}^{\hat{p}} \frac{\partial W(s, \alpha(s))}{\partial p} d s-\underbrace{[W(p, \alpha(\hat{p}))-W(\hat{p}, \alpha(\hat{p}))]}_{\int_{\hat{p}}^{p} \frac{\partial W(s, \alpha(\hat{p}))}{\partial p} d s} \\
& =\int_{\hat{p}}^{p} \frac{\partial W(s, \alpha(s))}{\partial p} d s-\int_{\hat{p}}^{p} \frac{\partial W(s, \alpha(\hat{p}))}{\partial p} d s \\
& =\int_{\hat{p}}^{p}\left[\frac{\partial W(s, \alpha(s))}{\partial p}-\frac{\partial W(s, \alpha(\hat{p}))}{\partial p}\right] d s
\end{aligned}
$$


Therefore, $\Delta(p, \hat{p}) \geq 0 \Leftrightarrow \int_{\hat{p}}^{p}\left[\frac{\partial W(s, \alpha(s))}{\partial p}-\frac{\partial W(s, \alpha(\hat{p}))}{\partial p}\right] d s \geq 0$. A more compact form of the same IC condition is

$$
\int_{\hat{p}}^{p}\left[\frac{\partial W(s, \alpha(s))}{\partial p}-\frac{\partial W(s, \alpha(\hat{p}))}{\partial p}\right] d s \geq 0 \Leftrightarrow \int_{\hat{p}}^{p}\left[\int_{\hat{p}}^{s} \frac{d}{d t}\left[\frac{\partial W(s, \alpha(t))}{\partial p}\right]_{t=u} d u\right] d s \geq 0 .
$$

The term inside the integral must be weakly positive, because the inequality must hold for any $\hat{p}, p$. When $\alpha(t)=\left(\alpha_{1}(t), \ldots, \alpha_{N}(t)\right)$, we have $\frac{d}{d t}\left[\frac{\partial W(s, \alpha(t))}{\partial p}\right]=\sum_{i=1}^{N} \frac{\partial W(s, \alpha(t))}{\partial p \partial \alpha_{i}} \alpha_{i}^{\prime}(t)$. Therefore, the condition implied by IC (analogous to Lemma 3 in the paper) is

$\sum_{i=1}^{N} \frac{\partial W(s, \alpha(t))}{\partial p \partial \alpha_{i}} \alpha_{i}^{\prime}(t)=\frac{\partial W(s, \alpha(t))}{\partial p \partial \alpha_{S}} \alpha_{S}^{\prime}(t)+\frac{\partial W(s, \alpha(t))}{\partial p \partial \alpha_{L}} \alpha_{L}^{\prime}(t)+\frac{\partial W(s, \alpha(t))}{\partial p \partial \alpha_{D}} \alpha_{D}^{\prime}(t) \geq 0, \quad$ for all $s, t$.

Thus, the monopolist solves problem (35) subject to condition (36). Once we find the allocation, we use (34) to compute the transfer.

Risk Neutrality. To contrast the problem under risk aversion with our baseline results under risk neutrality, consider first $\sigma=0$. In this case, condition (36) reduces simply to $\alpha_{D}(p)$ weakly increasing, and problem (35) reduces to $\max \int G(p, \alpha) d F(p)$, where

$$
G(p, \alpha)=W(p, \alpha)-K(p, \alpha)-\frac{\partial W(p, \alpha)}{\partial p}\left(\frac{1-F(p)}{f(p)}\right)
$$

and $h(p)=\frac{1-F(p)}{f(p)}$. Rather than solving this problem directly, we fix $p \in[c / d, 1]$ and we look for a contract that maximizes $G(p, \alpha)$ pointwise. It can be shown that any such contract satisfies $p \in\left(p^{* *}, p^{*}\right]$ or, equivalently,

$$
\theta \alpha_{S}-(p d-c)<(1-\theta)\left[c+c_{A}-\alpha_{L}-p \alpha_{D}\right]
$$

and

$$
p \alpha_{D} \leq \alpha_{S}+c+c_{A}-\alpha_{L}
$$

So the point-wise maximization reduces to maximize $G(p, \alpha)$ subject to (37) and (38).

We solve this problem in two steps: 
1. Define $\bar{p}$ as the solution to $p=h(p)$. 1 . When $p \leq \bar{p}$, the insurer sets $\alpha_{D}(p)=0, \alpha_{L}$ as large as possible, and $\alpha_{S}$ as small as possible, in the region where (38) and (37) are satisfied. It is easy so see that contract $\alpha(p)=\left(0, c_{A}, 0\right)$ satisfies all of this. Thus, this is the optimal menu in the region $p \leq \bar{p}$.

2. When $p>\bar{p}$, we solve the following linear optimization problem with linear constraints:

$$
\max _{\alpha_{S}, \alpha_{L}, \alpha_{D}} \alpha_{L}-\alpha_{S}+(p-h(p)) \alpha_{D}
$$

subject to

$$
\begin{aligned}
\alpha_{L}+p \alpha_{D} & \leq c+c_{A}+\frac{p d-c-\theta \alpha_{S}}{1-\theta} \\
\alpha_{L}+p \alpha_{D} & \leq c+c_{A}+\alpha_{S}
\end{aligned}
$$

It can be shown that the solution to this problem is contract $\alpha=\left(0, c_{A}, c / p\right)$.

The problem with this solution is that it violates the monotonicity constraint of $\alpha_{D}(p)$. Thus, we need to use ironing. Each types $p>\bar{p}$ will get the same allocation $\left(0, c_{A}, c / p^{*}\right)$, where $p^{*}$ is defined in Theorem 2 in the main text. ${ }^{31}$

Risk Aversion. We can follow similar steps in the case of risk aversion. Let $G_{R}(p, \alpha)$ be the analogous to $G(p, \alpha)$. First, from Proposition 7 , we know that we want to push $p^{* *}$ as low as possible to maximize $W-K$ pointwise. This argument is still true for $G_{R}(p, \alpha)$ because in the region where $p \leq p^{*}$ we have $G_{R}(p, \alpha)=W(p, \alpha)-K(p, \alpha)-\alpha_{S} h(p)$. Therefore, a contract $\alpha$ is dominated by $\hat{\alpha}$ if $\hat{p}^{* *}<p^{* *}, \hat{p}^{*}=p^{*}$ and $\hat{\alpha}_{D}=\alpha_{D}$, in the sense that $G_{R}(p, \alpha) \leq G_{R}(p, \hat{\alpha})$ for any $p$. This implies again that we can look for contracts where $\alpha_{S}=0$ or $\alpha_{L}=0$.

We proceed to maximize to find a contract $\alpha^{*}(p)$ such that $G_{R}(p, \alpha(p)) \geq G_{R}(p, \alpha)$ for any other contract $\alpha$. This solution is the optimal menu of contracts when incentive compatibility is not violated. It is not hard to see that for small values of $\sigma$, Equation 36 is equivalent to $\alpha_{D}(p)$ non-decreasing. We can understand qualitatively the shape of the optimal contract by looking at the contract $\alpha^{*}(p)$. Given that the problem is not analytically tractable, we solve it numerically.

\footnotetext{
${ }^{31}$ This is precisely the solution to the ironing problem.
} 

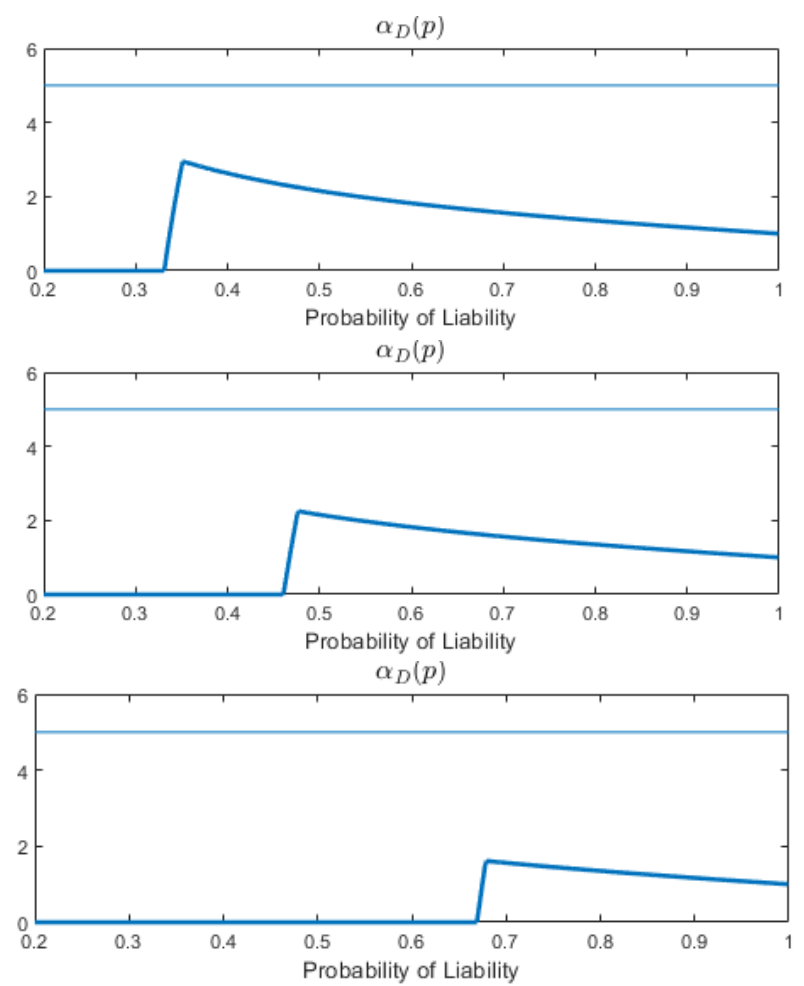

Figure 14: Damages coverage of contracts such that $G_{R}(p, \alpha(p)) \geq G_{R}(p, \alpha)$ for any other contract $\alpha$, for different distributions of types. The distribution of types in the plot at the top is $F(p)=p^{0.1}$, in the plot at middle is $F(p)=p$, and at the bottom is $F(p)=p^{5}$.

We simulated contracts for the parameters $c=c_{A}=1, d=5, \theta=0.8, \sigma=0.076$, and different distributions of types. The contract such that $G_{R}(p, \alpha(p)) \geq G_{R}(p, \alpha)$ features no settlement coverage and full litigation cost coverage, i.e., $\alpha_{S}(p)=0$ and $\alpha_{L}(p)=c_{A}$ for all p. However, the level of damages coverage changes with the distribution of types. Figure 14 shows the value of $\alpha_{D}(p)$ in the simulation of three scenarios.

Under risk-neutrality the highest type that is excluded is $\bar{p}$, which is the solution to $p=\frac{1-F(p)}{f(p)}$. When $F(p)=p^{a}$, this threshold corresponds to $\bar{p}=\left(\frac{1}{1+a}\right)^{\frac{1}{a}}$. In Figure 14, from top to bottom we have: $\bar{p}=0.37, \bar{p}=0.5$, and $\bar{p}=0.7$. The first observation is that risk aversion reduces the set of types that are excluded from damages insurance, i.e., $\bar{p}_{\text {risk averse }} \leq \bar{p}_{\text {risk neutral }}$.

Second, when types are not excluded, $\alpha_{D}(p)$ increases in a small region and decreases thereafter. Because incentive compatibility requires $\alpha_{D}(p)$ to be non-decreasing, there will be some threshold type $p_{\text {Iron }}$, with $p_{\text {Iron }} \geq \bar{p}_{\text {risk aversion }}$ such that any type $p \geq p_{\text {Iron }}$ receives the same damages coverage. Under risk neutrality, $p_{\text {Iron }}=\bar{p}_{\text {risk neutral }}$, so the optimal menu en- 
tailed only two contracts. Under risk aversion, however, we have more than two contracts: Low types do not get damages coverage; types that are a little bit higher than the highest excluded type buy small amount of damages coverage, specific to their types; high types, receive the same damages coverage, and it is not specific to their types.

Thus, qualitatively, the optimal contract under risk aversion features:

1. A contract that does not cover damages for types $p \leq \bar{p}_{\text {risk averse }}$.

2. A type-dependent contract that cover both damages and litigation costs for $p \in\left[\bar{p}_{\text {risk averse }}, p_{\text {Iron }}\right]$.

3. A type-independent contract that cover both damages and litigation costs for $p>p_{\text {Iron }}$.

The main qualitative differences between this contract and the case of risk neutrality is that there are fewer types excluded from damages insurance, and that the optimal menu features more than just two contracts. Finally, for larger levels of risk aversion, as in the case of symmetric information, the optimal contract may also cover settlements. This case is more complicated because the agent's willingness to pay may not be supermodular (see Figure 11).

\section{B.1.5 Perfect Competition Under Adverse Selection with Risk Aversion}

Proofs of the main results under risk neutrality rely on monotonicity of $W$ in $p$ and supermodularity of $W$ in $p$ and $\alpha_{D}$, as well as the unprofitability of insurance sold to types that

litigate. The assumption $\sigma<\frac{1}{d}$ ensures the monotonicity and supermodularity conditions, but does not guarantee unprofitability of insurance sold to types that litigate. For the latter, we need additional assumptions. For $p>p^{*}$, we can write

$$
W-K=\left(\frac{\sigma p(1-p)}{2}\right)\left[\alpha_{D}\left(2 d-\alpha_{D}\right)-\theta d^{2}\right]-\theta\left(c+c_{A}\right)
$$

This is never positive for all $p$, and may be negative for all $p$. Note that $W-K$ is increasing in $\alpha_{D}$, so imposing that $W-K$ is negative for all $p>p^{*}$ and $\alpha_{D}=d$ provides a sufficient condition for the insurer to lose money when the agent litigates. This condition corresponds to

$$
\sigma<\frac{8 \theta\left(c+c_{A}\right)}{d^{2}(1-\theta)}
$$


Note that this condition does not imply, and is not implied by, the assumption of $\sigma<\frac{1}{d}$. The simplest way to see this is to note that the constraint is impossible to meet for $\theta=0$ and is always met for $\theta=1$.

Under the assumption $\sigma<\min \left\{\frac{1}{d}, \frac{8 \theta\left(c+c_{A}\right)}{d^{2}(1-\theta)}\right\}$ our proofs (Lemma 1, Lemma 2, and Theorem 2 in the main text) go through essentially unchanged.

Lemma 1. This lemma states that no pooling equilibrium exists with a contract that yields $p^{*}<1$. In the risk-neutral analysis, we can restrict attention to the contract $\alpha_{L}=c_{A}$ and $\alpha_{D}=\frac{c}{p^{*}}$. The result holds because there is always a profitable alternative contract. Because $p^{*}<1$, the insurer incurs some costs on the types that litigate $p>p^{*}$. Hence, for this contract to earn zero profit for the insurer, it must be sold at a positive price. Let the break-even price, if all types buy this contract, equal $\tilde{P}$. Because of the supermodularity of $W$ in $p$ and $\alpha_{D}$, there exists an alternative contract with slightly lower $\alpha_{D}$ sold at a price slightly below $\tilde{P}$ that attracts just types that settle. Because the insurer would incur no costs from these types, the contract is profitable.

In the case of risk aversion, the analog to Lemma 1 holds easily if $\sigma<\frac{1}{d}$ so that the supermodularity of $W$ in $p$ and $\alpha_{D}$ continues to hold. While the basic logic is the same, a couple of things are different. First, we can still focus on the $p^{*}<1$ condition implied by a pooling contract, but note that we cannot restrict attention to the contract $\alpha_{L}=c_{A}$ and $\alpha_{D}=\frac{c}{p^{*}}$. The reason is that we lose pointwise maximization of $W$ - the contract $\alpha_{L}=c_{A}$ and $\alpha_{D}=\frac{c}{p^{*}}$ maximizes $W$ conditional on $p^{*}$ for types that settle, but $\alpha_{D}=d$ insurance maximizes $W$ conditional on $p^{*}$ for types that litigate. So with that change, it is necessary to consider any contract that yields $p^{*}$. But note that it remains true that the insurer incurs no costs on types that settle and incurs positive costs on types that litigate. Hence, for any $\alpha_{L}$ and $\alpha_{D}$ that yield cutoff $p^{*}$, the insurer must charge a positive price to break even. Because there is cross-subsidization, this price is less than the average cost from the types that litigate. And because of the supermodularity of $W$ in $p$ and $\alpha_{D}$, it remains possible to skim off the types that settle profitably.

Lemma 2. This lemma states that no separating equilibrium exists at all. Restrict attention to low- $\sigma$, where $W$ is monotone increasing in $p$ and $\alpha_{L}=c_{A}$ contracts dominate for types 
that settle. Also, consider the two-type case, $p \in\left\{p_{L}, p_{H}\right\}$.

To start, it is still true that there is no equilibrium with two contracts where the prices are both zero and all types settle, because types would not sort in that case. Under risk neutrality, the intuition is two-fold. First, because of pointwise maximization, any contract without $\alpha_{L}=c_{A}$ is dominated and cannot be part of an equilibrium. Second, because of single crossing, whichever $\alpha_{L}=c_{A}$ contract is preferred by one type will also be preferred by the other. With $\sigma<\frac{1}{d}$, pointwise maximization holds for types that settle and single-crossing holds generally. So with low $\sigma$, there is no separating equilibrium with two contracts at price 0 sold to types that settle.

So the remaining possibility includes at least one contract where types litigate. Let one such contract be $\alpha^{\text {Lit }}$, and let it be sold to type $p$ for $K\left(p, \alpha^{L i t}\right)>0$. From Proposition 2, we know that $W-K$ is maximized for insurance $\alpha^{I}$ such that $p^{*}\left(\alpha^{I}\right)=p$. Hence, type $p$ will always strictly prefer to pay $\varepsilon$ for $\alpha^{I}$ insurance as long as $\varepsilon$ is sufficiently close to 0 . And this is profitable if it attracts only type $p$ agents or if it attracts other less-costly agents. This rules out any candidate equilibrium where type $p_{H}$ litigates, because $\alpha^{I}$ that is such that $p^{*}\left(\alpha^{I}\right)=p_{H}$ surely attracts type $p_{H}$ agents, and any other agents that purchase $\alpha^{I}$ are of type $p_{L}$ and also would not litigate. So now the remaining possibility includes a contract where the type $p_{L}$ agent litigates, and another contract preferred by agent $p_{H}$. By our standard cream-skimming argument (which holds for $\sigma<\frac{1}{d}$ ), it is possible to alter the components of insurance and the price in such a way that attracts the $p_{L}$ types, induces settlement by them, and does not attract the $p_{H}$ agents.

With insurance possibly being profitable for types that litigate, the change is that such an insurance contract must be sold for $K(p, \alpha)$. But other than that, nothing really changes.

Now consider the continuous-density case. It remains true that two contracts that induce only settlement cannot form a separating equilibrium. It also remains true that any contract that induces some litigation is subject to cream-skimming. What would change is the cream-skimming need not necessarily just induce settlement. Candidate contracts that induce litigation must charge break-even average prices. That means lower- $p$ types may litigate but be less costly. They can be cream-skimmed. Any deviation that attracts lower- $p$ 
types attracts still-cheaper (on average) types, so altering the less-generous coverage down to cream skim cannot be made unprofitable by inducing selection away from the more-generous coverage. We can use the old proof until we reach the case where $\mu_{F}\left(D_{1}(S)\right)=0$. We cannot rule out such contracts with unprofitability. However, the same cream-skimming argument that the proof of Lemma 2 uses to rule out contracts that attract both types that litigate and types that settle can be used in the RA case. The reason is that $K(p, \alpha)$ is increasing in $p$. So any contract that attracts some measure of types that litigate, and no measure of types that settle, must sell at an average cost. If the types that buy the contract are $\underline{p}$ and

$\bar{p}$, then the price would need to be $\int_{\underline{p}}^{\bar{p}} K(p, \alpha) d f(p)$. But then the low- $p$ types can be sold less generous insurance for a lower price and be cream-skimmed away. For example, the $\underline{p}$ type could be sold perfect insurance for a positive price.

Theorem 2 follows from Lemmas 1 and 2, but the exact condition that guarantees that there is no profitable deviation from $p^{*}$ will now change. Thus, the qualitative result is the same, but the precise conditions to sustain an equilibrium are now different.

\section{B.2 Control over the Settlement Decision}

In this extension we consider the optimal assignment of control over the settlement process. In our main model we assumed that in general the agent decides whether to settle or litigate and negotiates the settlement, which is motivated by the features of actual liability insurance contracts that we observe in some industries, such as in patent litigation. In this framework the agent benefits from the ability to negotiate a better settlement with the third party, but the option to litigate gives rise to an ex post moral hazard problem. Instead, the agent and insurer may in some settings prefer an insurance contract whereby the insurer negotiates the settlement and controls the decision whether to settle or litigate, to avoid the problem of ex post moral hazard.

To study this problem, analogously to our main model, suppose that the insurer contracts with the agent, then observes $p$ and negotiates a settlement with the third party, under the threat of litigation. The insurer offers a contract $\alpha_{D} \in[0, d]$ to cover the possible damages that the agent may have to pay if found liable, as in the main body of the paper. Since the 
insurer controls the litigation process, it pays the settlement transfer and the litigation cost $c_{A}$; alternatively, this can also be modeled analogously as the agent paying the litigation cost, and the insurance contract covering (some part of) the litigation cost.

We assume, as in the literature on litigation insurance (Meurer (1992)), that the insurer must negotiate "in good faith," a restriction which in practice is interpreted to mean that the insurer must negotiate a settlement which maximizes I and A's joint payoff. Equivalently, this can also be seen as a requirement that the insurer must leave the agent no-worse-off than if it had not bought insurance. Under both of these interpretations, since $\alpha_{D}$ is a transfer between the agent and the insurer, the parties are indifferent over all $\alpha_{D}$. For generality, we also allow for the possibility that the insurer is better than the agent at negotiating a settlement: suppose the insurer has bargaining skill $\theta_{I}$, rather than $\theta$.

First, notice that this model of settlement is in fact analogous to our baseline model with no insurance: one party (in this case the insurer) negotiates a settlement to maximize I and A's joint payoff, which is equivalent to a model without insurance where the agent negotiates a settlement to maximize its own payoff, though possibly with different bargaining skill.

In this extension, the agent's payoff without insurance is

$$
\bar{V}=-c_{A}-p d+\theta\left(c+c_{A}\right) .
$$

The agent's payoff with insurance (where the insurer bargains) is

$$
V=-c_{A}-p d+\theta_{I}\left(c+c_{A}\right)
$$

So the agent and insurer's net joint surplus from insurance (relative to no insurance) is

$$
W=\left(\theta_{I}-\theta\right)\left(c_{A}+c\right)
$$

It is clear that such insurance cannot be profitable if $\theta>\theta_{I}$, so we will focus on the case where $\theta_{I} \geq \theta$. Also, notice that this surplus is independent of $p$ : all types value this kind of insurance contract by the same amount. With a monopolist insurer, the optimal price 
of this insurance is $W$, whereas with competition it is 0 . In both settings the bargaining surplus is always positive, so there is never any litigation in equilibrium. Moreover, because this surplus is independent of $p$, the joint surplus from such insurance is the same across different market and information structures. Whether $p$ is the agent's private information or not at the time of contracting with the insurer is in fact irrelevant in this case-both parties anticipate that at the time of bargaining, I knows $p$ and bargains to maximize A and I's joint payoff (which is analogous to our baseline model where A bargains without insurance). A receives no information rents, since the net joint surplus from this insurance contract is independent of $p$.

For each market structure and information structure, we can now compare the insurer's overall profit in our main model against its profit from selling insurer-controlled insurance. We mainly focus on the cases where setting $p^{*}=1$ is optimal, although analogous comparisons and intuitions emerge in all cases, where $p^{*}<1$ may be optimal.

\section{Monopoly and competition under symmetric information}

To begin, consider the setting with symmetric information. We show that under both monopoly and perfect competition, there exists a threshold bargaining parameter $\widetilde{\theta}_{I}$ such that it is optimal to assign the right to settle to the agent for any $\theta_{I} \leq \widetilde{\theta}_{I}$, and to assign it to the insurer when $\theta_{I}>\widetilde{\theta}_{I}$. Moreover, for $\theta_{I}=\theta$, agent-controlled settlement is optimal, confirming that the results in our main model are robust.

From Proposition 4, we can compare a monopolist insurer's profit from agent-controlled contracts, as well as a perfectly competitive insurer's profit from agent-controlled contractsboth of these relate to the net joint surplus of insurance. In the case where $p^{*}=1$ is optimal, we have

$$
P_{M}(1)=J S_{C}(1)=E_{p}(W(p, 1))=\int_{\frac{c}{d}}^{1}(1-\theta)\left(c_{A}+c p\right) d F(p) .
$$

We compare this to the I's profit and the net joint surplus from insurer-controlled contracts:

$$
\tilde{P}_{M}=W_{C} \equiv \int_{\frac{c}{d}}^{1}\left(\theta_{I}-\theta\right)\left(c_{A}+c\right) d F(p) .
$$


With some rearranging, we have that

$$
\widetilde{P}_{M} \geq P_{M}(1)=J S_{C}(1) \quad \Leftrightarrow \quad \int_{\frac{c}{d}}^{1}(1-\theta) c(1-p) d F(p) \geq \int_{\frac{c}{d}}^{1}\left(1-\theta_{I}\right)\left(c_{A}+c\right) d F(p)
$$

Notice that for $\theta_{I}$ sufficiently high (e.g. $\theta_{I}=1$ ), the right-hand side is 0 and the left-hand side is positive (independent of $\theta_{I}$ ), so insurer-controlled insurance is optimal. On the other hand, for $\theta_{I}$ low enough (e.g. $\left.\theta_{I}=\theta\right)$, we have $c(1-p)<c+c_{A}$, so the inequality is reversed, hence agent-controlled insurance is optimal. There exists a unique threshold $\widetilde{\theta}_{I}$ given by

$$
\int_{\frac{c}{d}}^{1}(1-\theta) c(1-p) d F(p)=\int_{\frac{c}{d}}^{1}\left(1-\widetilde{\theta}_{I}\right)\left(c_{A}+c\right) d F(p)
$$

such that for $\theta_{I}>\widetilde{\theta}_{I}$, insurer-controlled contracts are optimal, whereas for $\theta_{I} \leq \widetilde{\theta}_{I}$, agentcontrolled contracts are optimal. Moreover, when the agent and insurer are equally good at bargaining, i.e. $\theta=\theta_{I}$, agent-controlled insurance contracts are optimal. Our equilibrium results from the main model continue to hold when $\theta_{I}$ and $\theta$ are similar enough.

\section{Competition under private information}

Now consider a competitive market where the agent is privately informed about its type. To see whether agent-controlled or insurer-controlled insurance will be sustained as an equilibrium, we must again compare the insurer and agent's net joint surplus from each type of contract. From Lemma 2 and Theorem 2, the only possible agent-controlled equilibrium contract is a pooling contract with $p^{*}=1$. I and A's joint surplus is

$$
J S_{C}(1) \equiv E_{p}(W(p, 1))=\int_{\frac{c}{d}}^{1}(1-\theta)\left(c_{A}+c p\right) d F(p)
$$

With an insurer-controlled insurance contract, I and A's joint surplus is

$$
\widetilde{P}_{M}=W_{C}=\int_{\frac{c}{d}}^{1}\left(\theta_{I}-\theta\right)\left(c_{A}+c\right) d F(p)
$$

Both of these are identical to the case of symmetric information, and thus our conclusions coincide: for $\theta_{I}>\widetilde{\theta}_{I}$, insurer-controlled contracts are offered in equilibrium, whereas for $\theta_{I} \leq \widetilde{\theta}_{I}$, agent-controlled contracts are offered in equilibrium. When the agent and insurer 
have equal (or similar enough) bargaining skill, our equilibrium results from the main model continue to hold.

\section{Monopoly under private information}

Finally, consider the monopoly setting with private information. In the case where $p^{*}=1$ is optimal, from Theorem 1, the insurer offers a menu of two contracts: a contract with $\alpha_{D}=0$ sold at price $(1-\theta) c_{A}$, for types $p \leq \bar{p}$, and one with $\alpha_{D}=\frac{c}{p^{*}}$ sold at price $(1-\theta)\left(c_{A}+c \frac{\bar{p}}{p^{*}}\right)$, for types $p>\bar{p}$. The insurer's total revenue here is

$$
R_{M}(1) \equiv \int_{\frac{c}{d}}^{\bar{p}}(1-\theta) c_{A} d F(p)+\int_{\bar{p}}^{1}(1-\theta)\left(c_{A}+c \frac{\bar{p}}{p^{*}}\right) d F(p)
$$

We compare this against the insurer's profit in this extension:

$$
\widetilde{P}_{M}=\int_{\frac{c}{d}}^{1}\left(\theta_{I}-\theta\right)\left(c_{A}+c\right) d F(p) .
$$

So we have

$$
\begin{aligned}
\widetilde{P}_{M} \geq R_{M}(1) \Leftrightarrow & \int_{\frac{c}{d}}^{\bar{p}}\left(\theta_{I}-\theta\right) c d F(p)+\int_{\bar{p}}^{1}\left(\theta_{I}-\theta\right) c\left(1-\frac{\bar{p}}{p^{*}}\right) d F(p) \geq \\
& \geq \int_{\frac{c}{d}}^{\bar{p}}\left(1-\theta_{I}\right) c_{A} d F(p)+\int_{\bar{p}}^{1}\left(1-\theta_{I}\right)\left(c_{A}+c \frac{\bar{p}}{p^{*}}\right) d F(p)
\end{aligned}
$$

As before, for $\theta_{I}$ sufficiently high (e.g. $\theta_{I}=1$ ), the right-hand side is 0 and the left-hand side is positive, so insurer-controlled insurance is optimal. On the other hand, for $\theta_{I}$ low enough (e.g. $\theta_{I}=\theta$ ), the left-hand side is 0 while the right-hand side is positive, so the inequality is reversed, hence agent-controlled insurance is optimal. There exists a threshold $\bar{\theta}_{I}$ given by the expression

$\int_{\frac{c}{d}}^{\bar{p}}\left(\bar{\theta}_{I}-\theta\right) c d F(p)+\int_{\bar{p}}^{1}\left(\bar{\theta}_{I}-\theta\right) c\left(1-\frac{\bar{p}}{p^{*}}\right) d F(p)=\int_{\frac{c}{d}}^{\bar{p}}\left(1-\bar{\theta}_{I}\right) c_{A} d F(p)+\int_{\bar{p}}^{1}\left(1-\bar{\theta}_{I}\right)\left(c_{A}+c \frac{\bar{p}}{p^{*}}\right) d F(p)$

such that for $\theta_{I}>\bar{\theta}_{I}$, insurer-controlled contracts are optimal, whereas for $\theta_{I} \leq \bar{\theta}_{I}$, agentcontrolled contracts are optimal. As in the setting with symmetric information, the results from our main model continue to hold as long as $\theta_{I}$ and $\theta$ are similar enough. 


\section{B.3 Bargaining under Incomplete Information}

When parties bargain under incomplete information, generically litigation arises in equilibrium. In the main text, when parties bargain under complete information, litigation never occurs in equilibrium. This is a well-known difference between these two models of bargaining, independent of the issue of insurance. In our baseline setting, insurance has the potential of inducing litigation in an environment that otherwise would never feature litigation.

For illustrative purposes, we consider the two-type case: A fraction $\lambda$ of agents are type $p_{H}$ and a fraction $1-\lambda$ are type $p_{L}$, with $0 \leq p_{L}<p_{H} \leq 1$. Assume the agent is protected by the liability insurance policy $\alpha=\left(\alpha_{S}, \alpha_{L}, \alpha_{D}\right)$. Following the literature, we assume the uninformed party makes a take-it-or-leave it offer to the informed party. Consider the following two offers:

$$
\begin{aligned}
S^{L} & =\alpha_{S}+\left(c_{A}-\alpha_{L}\right)+p^{L}\left(d-\alpha_{D}\right) \\
S^{H} & =\alpha_{S}+\left(c_{A}-\alpha_{L}\right)+p^{H}\left(d-\alpha_{D}\right)
\end{aligned}
$$

The low-risk type is indifferent between paying $S^{L}$ and litigating, while the high-risk agent is strictly better off by accepting $S^{L}$. The settlement offer $S^{H}$ leaves the high-risk type indifferent between accepting the offer or litigation but low-risk type rejects the offer and litigate. The third party's outside option is $E[p] d-c$ because it can always make a 'bad faith' settlement offer $\left(S^{\infty}=+\infty\right)$ that forces both types to litigate. We have three cases:

1. TP makes offer $S^{L}$, both types of agents settle, and TP's payoff is

$$
\pi_{T P}\left(S^{L}\right)=\alpha_{S}+\left(c_{A}-\alpha_{L}\right)+p^{L}\left(d-\alpha_{D}\right)
$$

2. TP makes offer $S^{H}$, high-risk types settle but low-risk type litigate, and TP's payoff is

$$
\pi_{T P}\left(S^{H}\right)=\lambda\left[\alpha_{S}+\left(c_{A}-\alpha_{L}\right)+p^{H}\left(d-\alpha_{D}\right)\right]+(1-\lambda)\left[p^{L} d-c\right]
$$


3. TP forces litigation by offering $S^{\infty}$ and TP's payoff in this case is

$$
\pi_{T P}\left(S^{\infty}\right)=\left(\lambda p^{H}+(1-\lambda) p^{L}\right) d-c
$$

It can be shown that

$$
\begin{aligned}
& \pi_{T P}\left(S^{H}\right)=\pi_{T P}\left(S^{\infty}\right)+\underbrace{\lambda\left(c+c_{A}-\alpha_{L}-p^{H} \alpha_{D}+\alpha_{S}\right)}_{\equiv Y(\alpha)} \\
& \pi_{T P}\left(S^{H}\right)=\pi_{T P}\left(S^{L}\right)+\underbrace{\lambda\left(p^{H}-p^{L}\right)\left(d-\alpha_{D}\right)-(1-\lambda)\left[c+c_{A}-\alpha_{L}-p^{L} \alpha_{D}+\alpha_{S}\right]}_{\equiv Z(\alpha)} \\
& \pi_{T P}\left(S^{L}\right)=\pi_{T P}\left(S^{\infty}\right)+\underbrace{Y(\alpha)-Z(\alpha)}_{\equiv W(\alpha)}
\end{aligned}
$$

The optimal offer, is determined by $Y(\alpha), Z(\alpha)$, and $W(\alpha)$, because $S^{H} \succeq S^{L}$ is equivalent to $Y(\alpha) \geq 0 ; S^{H} \succeq S^{\infty}$ is equivalent to $Z(\alpha) \geq 0$; and $S^{L} \succeq S^{\infty}$ is equivalent to $W(\alpha) \geq 0$. In fact, the optimal offer is:

1. $S^{L}(\alpha)$ if and only if $\alpha \in C^{L}=\{\alpha: Z(\alpha) \leq 0$ and $Y(\alpha) \geq Z(\alpha)\}$.

2. $S^{H}(\alpha)$ if and only if $\alpha \in C^{H}=\{\alpha: Z(\alpha) \geq 0$ and $Y(\alpha) \geq 0\}$.

3. $S^{\infty}$ if and only if $\alpha \in C^{\infty}=\{\alpha: Y(\alpha) \leq 0$ and $Y(\alpha) \leq Z(\alpha)\}$.

Without insurance (by setting $\left.\alpha_{S}=\alpha_{L}=\alpha_{D}=0\right)$ we have $Y(0)=\lambda\left(c+c_{A}\right)>0$ and $Z(0)=\lambda\left(p^{H}-p^{L}\right) d-(1-\lambda)\left(c+c_{A}\right)$, so it is optimal to offer $S^{L}(0)$ if $Z(0)<0$, or to offer $S^{H}$ if $Z(0)>0$. Thus, it is possible to obtain litigation in equilibrium without insurance.

We now derive the agent's willingness to pay for insurance policy $\alpha$. We first consider the case $Z(0)<0$ or, equivalently, $\left(\frac{\lambda}{1-\lambda}\right)\left(p^{H}-p^{L}\right) d<c+c_{A}$. In this case, the optimal settlement offer for an agent without insurance is $S^{L}(0)$. Hence, every agent gets the same outside option from not buying insurance, which is to pay $S^{L}(0)$ as a settlement fee.

The willingness to pay of an agent of type $p^{L}$ pay for insurance contract $\alpha$ is:

- $S^{L}(0)-\left(S^{L}(\alpha)-\alpha_{S}\right)=\alpha_{L}+p^{L} \alpha_{D}$ if $\alpha \in C^{L}$. 
- $S^{L}(0)-\left[\left(c_{A}-\alpha_{L}\right)+p^{L}\left(d-\alpha_{D}\right)\right]=\alpha_{L}+p^{L} \alpha_{D}$ if $\alpha \notin C^{L}$.

This is because the low-type agent only accepts $S^{L}(\alpha)$ and rejects (and goes to litigation) with any other offer.

The willingness to pay of an agent of type $p^{H}$ pay for insurance contract $\alpha$ is:

- $S^{L}(0)-\left(S^{L}(\alpha)-\alpha_{S}\right)=\alpha_{L}+p^{L} \alpha_{D}$ if $\alpha \in C^{L}$.

- $S^{L}(0)-\left(S^{H}(\alpha)-\alpha_{S}\right)=\alpha_{L}+p^{H} \alpha_{D}-d\left(p^{H}-p^{L}\right)$ if $\alpha \in C^{H}$.

- $S^{L}(0)-\left[\left(c_{A}-\alpha_{L}\right)+p^{H}\left(d-\alpha_{D}\right)\right]=\alpha_{L}+p^{H} \alpha_{D}-d\left(p^{H}-p^{L}\right)$ otherwise.

Conditional on having bought insurance policy $\alpha$, the high-type agent accepts both offers $S^{L}(\alpha)$ and $S^{H}(\alpha)$, and rejects $S^{\infty}$.

\section{Complete Information between the insurer and the agent}

If the agent's type is $p^{L}$, the insurer would offer a policy $\alpha \in C^{L}$ such that maximizes $\alpha_{L}+p^{L} \alpha_{D}-\alpha_{S}$. This is because under this policy the agent settles so the insurer has to pay $\alpha_{S}$, but the insurer does not pay $\alpha_{L}+p^{L} \alpha_{D}$ (this is the gain from an improved bargaining position). In contrast, if the agent litigates, the insurer pays all the cost so the net surplus between the insurer and the agent is zero.

Thus, the optimal contract solves

$$
\max _{\left(\alpha_{S}, \alpha_{L}, \alpha_{D}\right)} \alpha_{L}+p^{L} \alpha_{D}-\alpha_{S}
$$

subject to

$$
\begin{aligned}
(1-\lambda)\left[\alpha_{L}-\alpha_{S}\right]+\alpha_{D}\left(p^{L}-\lambda p^{H}\right) & \leq(1-\lambda)\left(c+c_{A}\right)-\lambda d\left(p^{H}-p^{L}\right) \\
\alpha_{L}-\alpha_{S}+\alpha_{D} & \leq c+c_{A}-\lambda d\left(p^{H}-p^{L}\right)
\end{aligned}
$$


We can treat $\alpha_{L}-\alpha_{S}$ as a new variable $x \in\left(-\infty, c_{A}\right]$ and transform the problem to:

$$
\max _{\left(x, \alpha_{D}\right)} x+p^{L} \alpha_{D}
$$

subject to

$$
\begin{aligned}
(1-\lambda) x+\alpha_{D}\left(p^{L}-\lambda p^{H}\right) & \leq(1-\lambda)\left(c+c_{A}\right)-\lambda d\left(p^{H}-p^{L}\right) \\
x+\alpha_{D} & \leq c+c_{A}-\lambda d\left(p^{H}-p^{L}\right)
\end{aligned}
$$

The solution to this problem is easy to compute, although it depends on the parameters of the problem. For example if $p^{H}<\lambda\left(1-p^{H}\right)$, the solution is $\alpha_{D}=0$ and $x^{*}=c+c_{A}-$ $\left(\frac{\lambda}{1-\lambda}\right)\left(p^{H}-p^{L}\right) d$, which is positive by the assumption $Z(0)<0$. In other words, under some assumptions on the parameters, the optimal contract under complete information for an agent of type $p^{L}$ is to set $\alpha_{L}-\alpha_{S}=x^{*}$.

Similar to the baseline case, under complete information the insurer sells a contract that never induces litigation. In contrast to the baseline case, in this setting insurance improves welfare by reducing the amount of litigation in equilibrium. Also, as in the baseline case, there is multiplicity in the optimal contract.

\section{Incomplete and Symmetric Information}

Under incomplete and symmetric information between the agent and the insurer, the insurer would induce litigation by offering $\alpha \in C^{\infty}$, incurring in losses, so this cannot be optimal. When $\alpha \in C^{H}$, the insurer induces litigation for the low-type. In this case, The net surplus between the low-type agent and the insurer is zero, whereas the net surplus between the high-type agent and the insurer is $\alpha_{L}-\alpha_{S}+p^{H} \alpha_{D}-d\left(p^{H}-p^{L}\right)$. Finally, when $\alpha \in C^{L}$, the net surplus between both types of agents and the insurer is $\alpha_{L}-\alpha_{S}+p^{L} \alpha_{D}$.

As in the case of complete information, we can see that only the difference between $\alpha_{L}$ and $\alpha_{S}$ is relevant for the insurer's problem. Thus, the optimal contract under incomplete and 
symmetric information solves:

$$
\max _{\alpha=\left(\alpha_{S}, \alpha_{L}, \alpha_{D}\right)}\left[x+p^{L} \alpha_{D}\right] 1\left(\alpha \in C^{L}\right)+\lambda\left[x+p^{H} \alpha_{D}-d\left(p^{H}-p^{L}\right)\right] 1\left(\alpha \in C^{H}\right)
$$

where $x=\alpha_{L}-\alpha_{S}$. The solution to this optimization problem depends on the parameters of the problem. In contrast to the baseline case in the main text, where $\alpha_{S}=0$ and $\alpha_{L}=c_{A}$, when parties bargain under incomplete information we obtain multiple optimal contracts, because $\alpha_{S}$ and $\alpha_{L}$ are "perfect substitutes."

In this setting, the insurer may not want to minimize the difference $\alpha_{S}-\alpha_{L}$, which in the main text leads to set $\alpha_{S}=0$ and $\alpha_{L}=c_{A}$, because the insurer chooses a contract to extract rent from the third party, by inducing it to make a low offer that is accepted by the agent, thus raising the agent's willingness to pay for insurance.

\section{B.4 Alternative Equilibrium Concepts}

One of the criticisms of the results in Rothschild and Stiglitz (1976) is that, under some conditions, equilibrium fails to exist. In our setting, when the mass of high-risk types is sufficiently low equilibrium may also fail to exist. The literature following Rothschild and Stiglitz (1976) has come up with alternative equilibrium notions under which an equilibrium exists. Riley (1979) proposes a reactive equilibrium notion, which exists in the Rothschild and Stiglitz (1976) setting. In our setting, in contrast, this equilibrium may not exist. First, if there exists a Nash equilibrium set of contracts, it is a Riley equilibrium. Consider the case where there is no Nash equilibrium. Then, there exists a contract $p_{d}^{*}<1$ that is a profitable deviation from $p^{*}=1$. But then, this new contract can be cream-skimmed. And the contract that cream-skims $p_{d}^{*}$ can never be "safe," i.e., it can also be cream-skimmed by another contract. Therefore in our setting Riley and Nash coincide, meaning that a Riley equilibrium may not exist.

Azevedo and Gottlieb (2017) propose an equilibrium refinement of the free-entry equilibrium (there is multiplicity of free-entry equilibria in the Rothschild and Stiglitz (1976) setting). This equilibrium notion requires continuity in the costs. In our setting, however, the insurer 
costs are not continuous as a function of the agent's type, so we cannot directly use this equilibrium notion. Another alternative is to use mixed strategies. Farinha Luz (2017) characterizes a mixed-strategy equilibrium in the Rothschild and Stiglitz (1976) setting. The main assumption is that there is a finite number of firms, and most of the analysis is for the case of two firms. ${ }^{32}$ We believe it may be possible to construct a mixed-strategy equilibrium in our setting, but this is beyond the scope of this paper, as we focus on pure strategies, which are more natural to interpret in our contracting environment.

Wilson (1977) proposes the notion of anticipatory equilibrium. A set of policies is a Wilson equilibrium if each policy earns nonnegative profits and there is no other set of policies which earn positive profits in the aggregate and nonnegative profits individually, after the unprofitable policies in the original set have been withdrawn. When a Nash equilibrium exists, this is also a Wilson equilibrium, because there are no profitable deviations. When a Nash equilibrium does not exists, a Wilson equilibrium may exist, and consists of pooling both types into a single contract: the contract that generates zero joint profits, and it is the most preferred contract for the low-risk type, i.e., the contract that targets the low type $p^{*}=p_{L}$, sold at price

$$
Q=\lambda\left(c_{A}+c \frac{p_{H}}{p_{L}}\right) .
$$

\section{B.5 Characterization of the Symmetric Information Contract}

To help characterize the solution to this problem, we consider smooth distributions for which the density may equal zero only at the boundaries of the support. ${ }^{33}$

Assumption 3. Let $F(\cdot)$ be twice-continuously differentiable, with probability density $f(p)>$ 0 for all $p \in(0,1)$.

\footnotetext{
${ }^{32}$ The paper also discusses the case of the number of firms going to infinity.

${ }^{33}$ These formulas also apply to the case where $p$ has a discrete distribution with binary support, which is available upon request.
} 
Consider the derivative of the objective function in equation (11):

$$
\Psi_{S I}^{\prime}\left(p^{*}\right)=\underbrace{(1-\theta)\left(c+c_{A}\right) f\left(p^{*}\right)}_{\text {marginal type }}-\underbrace{(1-\theta) \frac{c}{\left(p^{*}\right)^{2}} \int_{c / d}^{p^{*}} p d F(p)}_{\text {infra-marginal types }}+\underbrace{\theta\left(c+c_{A}\right) f\left(p^{*}\right)}_{\text {marginal type }} .
$$

Increasing coverage has an effect on the marginal type and infra-marginal types. First, the marginal type $p^{*}$ gets perfect insurance and extracts the full bargaining surplus $\left(c+c_{A}\right)$ from the third party. The marginal gain of increasing $p^{*}$ is shown in equation (44) in two different places: a gain of $(1-\theta)\left(c+c_{A}\right)$ from the leverage of types marginally below type $p^{*}$; and a gain from avoiding a loss of $\theta\left(c+c_{A}\right)$ in bargaining surplus for types marginally above $p^{*}$ who would settle instead of going to court. Second, the infra-marginal types $p<p^{*}$ receive a level of insurance further away from their perfect level, inducing a loss in the joint surplus of the insurer and agent.

The optimal contract either precludes litigation entirely $\left(p^{*}=1\right)$ or balances the gain of the marginal type versus the average loss of the infra-marginal types. To further understand when it is optimal to offer a contract that induces litigation, we define the elasticity of density.

Definition 2. For distributions satisfying Assumption 3, the elasticity of density is

$$
\eta(p)=\frac{p f^{\prime}(p)}{f(p)}
$$

It is easy to see that the following identity holds

$$
\Psi_{S I}^{\prime \prime}(p) p^{2}+2 \Psi_{S I}^{\prime}(p) p=\frac{p f(p)}{c_{A}+c}\left[\eta(p)+1+\frac{c_{A}+\theta c}{c_{A}+c}\right]
$$

Thus, if $p^{*}$ is an interior solution of problem (11), the first and second order conditions, $\Psi_{S I}^{\prime}\left(p^{*}\right)=0$ and $\Psi_{S I}^{\prime \prime}\left(p^{*}\right)<0$, respectively, imply

$$
\eta\left(p^{*}\right)<-\left(1+\frac{c_{A}+\theta c}{c_{A}+c}\right)
$$

The elasticity of density provides us with a sufficient condition for a unique solution of problem (11). 
Lemma 13. Under Assumption 3, the solution to problem (11) is unique and equal to $p^{*}=1$ if for all $p \in\left[\frac{c}{d}, 1\right]$ we have

$$
\eta(p) \geq-\left(1+\frac{c_{A}+\theta c}{c_{A}+c}\right)
$$

For any convex distribution $F(\cdot), \eta(p) \geq 0$ for all $p$. By Lemma 13, the unique optimal contract precludes litigation by setting $p^{*}=1$. When the density function is increasing, the marginal gain dominates the infra-marginal loss, i.e., it is suboptimal to sell insurance generous enough to induce litigation by risky types. Intuitively, it is also optimal to preclude litigation when $F(p)$ is mildly concave.

There are many distributions where the solution to (11) induces litigation for some types. In such cases, $\eta(p)$ allows us to provide a sufficient condition for uniqueness.

Lemma 14. Under Assumption 3, let $p^{*}<1$ be such that $\Psi_{S I}^{\prime}\left(p^{*}\right)=0$ and $\Psi_{S I}^{\prime \prime}\left(p^{*}\right)<0$. Then, $p^{*}$ is the unique interior solution if

$$
\eta(p) \leq-\left(1+\frac{c_{A}+\theta c}{c+c_{A}}\right), \text { for all } p \in\left[p^{*}, 1\right]
$$

When $p^{*}<1$, the insurer targets a particular type $p^{*}$ with perfect insurance and induces litigation by types $p>p^{*}$ and imperfect insurance for types $p<p^{*}$. In targeting, the insurer seeks a sufficiently low level of relative litigation risk associated with type $p^{*} \cdot{ }^{34}$ When the elasticity of density falls with $p$ and the density of a high-risk type is low, ${ }^{35}$ intuitively, the insurer prefers to induce some litigation. We have the following result.

Corollary 1. If $\eta(p)$ is non-increasing and $f(1)<\frac{(1-\theta) c}{c_{A}+c} \int_{c / d}^{1} p d F(p)$, there exists a unique $p^{*} \in\left(\frac{c}{d}, 1\right)$ that solves (11).

Proof. When $\Psi_{S I}^{\prime}(1)<0$, there exists $p^{*}<1$ that solves $(11)$. Since $\eta\left(p^{*}\right)<-\left(1+\frac{c_{A}+\theta c}{c+c_{A}}\right)$ and $\eta(p)$ is non-increasing, the sufficient condition for uniqueness in Lemma 14 holds.

\footnotetext{
${ }^{34} \eta(\cdot)$ is analogous to the Arrow-Pratt coefficient of relative risk aversion when the Bernoulli utility function is $u(x) \equiv F(x)$. A large coefficient of relative risk aversion implies that the decision-maker has very little to gain by gambling. In our environment, a large negative $\eta(p)$ means that the insurer wants a lower $p$, because it has very little to lose from gambling on relatively unlikely litigation.

${ }^{35}$ Note that specifying $\eta(p)$ as decreasing in $p$ is a weaker assumption than specifying $f(p)$ to have decreasing density and to be log-concave in $p$.
} 
Next, we present comparative statics results. ${ }^{36}$

Lemma 15. $p^{*}$ is non-decreasing in $c_{A}$ and $\theta$, and is non-increasing in $d$.

Lemma 15 follows from the Topkis monotonicity theorem. An increase in the agent's litigation $\operatorname{cost} c_{A}$ increases the opportunity cost of litigation. The gain from increasing the number of types that settle is unambiguously higher, so $p^{*}$ is non-decreasing in $c_{A}$. An increase in the agent's bargaining skill decreases the insurer's ability to profit from insurance: the willingness to pay for insurance falls but the cost of insurance is the same. Thus $p^{*}$ is non-decreasing in $\theta$ because an increase in the agent's bargaining skill does not change the surplus gain of the marginal type, but it reduces the surplus loss of the infra-marginal types. An increase in damages $d$ increases the number of agents exposed to credible liability claims. Thus the number of infra-marginal types increases and therefore $p^{*}$ weakly decreases. The effect of the third-party's litigation cost $c$ is ambiguous, because it increases both the surplus gain of the marginal type and the loss in surplus of the infra-marginal types.

\section{Proof of Lemma 13}

Proof. $p^{*} \neq \hat{p}^{\prime}>1$ and $p^{*} \neq \frac{c}{d}$ because $\Psi_{S I}\left(\hat{p}^{\prime}\right)<\Psi_{S I}(1)$ and $\Psi_{S I}\left(\frac{c}{d}\right)<\Psi_{S I}(1)$. With a continuous distribution $F(\cdot)$, the objective function is continuous, so a maximum exists (not necessarily unique). With a continuous density, the derivative of the $\Psi_{S I}(\cdot)$ is also continuous. If there are multiple solutions, then at least one must be an interior local maximum. The density $f(\cdot)$ is differentiable because $F$ is twice differentiable, so the first and second order conditions imply

$$
\left(c+c_{A}\right) f^{\prime}\left(p^{*}\right)+\frac{f\left(p^{*}\right)}{p^{*}}\left[2 c_{A}+(1+\theta) c\right]<0 .
$$

Then, if for all $p^{*}$ condition (45) is violated, we can guarantee that the solution of the problem is $p^{*}=1$ because in that case there is no interior local maximum of $\Psi(\cdot)$. Hence, since a solution must exist, it must be that $p^{*}=1$.

\footnotetext{
${ }^{36}$ As the two-type case suggests, problem (11) may have multiple solutions, e.g. with a continuous distribution with non-monotonic $\eta(p)$. If so, the monotonicity of $p^{*}$ is in the strong set order.
} 


\section{Proof of Lemma 14}

Proof. Suppose $p_{1}<p_{2}<1$ are two points satisfying the FOC, $\Psi_{S I}^{\prime}\left(p_{i}\right)=0$, and the SOC, $\Psi_{S I}^{\prime \prime}\left(p_{i}\right)<0$. We have $p_{i}>\frac{c}{d}$ because $\Psi_{S I}^{\prime}\left(\frac{c}{d}\right)>0$. Then, by continuity of $\Psi^{\prime}$, there exists $\xi \in\left(p_{1}, p_{2}\right)$ such that $\Psi_{S I}^{\prime}(\xi)=0$ and $\Psi_{S I}^{\prime \prime}(\xi)>0$, which implies

$$
\left(c+c_{A}\right) f^{\prime}(\xi)+\frac{f(\xi)}{\xi}\left[2 c_{A}+(1+\theta) c\right]>0 \Leftrightarrow \eta(\xi)>-1-\frac{c_{A}+\theta c}{c_{A}+c} .
$$

If this condition does not hold, the existence of both $p_{1}$ and $p_{2}$ is a contradiction.

\section{Proof of Lemma 15}

Proof. By Topkis' monotonicity theorem, $\frac{\partial^{2} \Psi_{S I}}{\partial \hat{p} \partial \eta} \geq 0 \Rightarrow p^{*}(\cdot)$ non-decreasing in $\eta$. It is easy to show that $\frac{\partial^{2} \Psi_{S I}}{\partial \hat{p} \partial c_{A}}>0, \frac{\partial^{2} \Psi_{S I}}{\partial \hat{p} \partial \theta}>0$, and $\frac{\partial^{2} \Psi_{S I}}{\partial \hat{p} \partial d}<0$. We have $\frac{\partial^{2} \Psi_{S I}}{\partial \hat{p} \partial c}\left(p^{*}\right)=f\left(p^{*}\right)-$ $\frac{(1-\theta)}{\left(p^{*}\right)^{2}}\left[\int_{c / d}^{p^{*}} p f(p) d p-\left(\frac{c}{d}\right)^{2} f\left(\frac{c}{d}\right)\right]$. As $p^{*} \rightarrow \frac{c}{d}, \frac{\partial^{2} \Psi_{S I}}{\partial \hat{p} \partial c} \rightarrow \theta f\left(\frac{c}{d}\right)>0$. Moreover, $\frac{\partial^{2} \Psi_{S I}}{\partial \hat{p} \partial c}$ is increasing if $\eta(p) \geq-1$. 